\title{
Pólya's Permanent Problem
}

\author{
William McCuaig* \\ 5268 Eglinton St. \\ Burnaby, British Columbia, Canada \\ V5G 2B2
}

Submitted: Dec 5, 1998; Accepted: Feb 17, 2004; Published: Nov 6, 2004

MR Subject Classifications: Primary 05C20

Secondary 05-02, 05A15, 05C15, 05C38, 05C70, 05C75, 05C83,15A15

\begin{abstract}
A square real matrix is sign-nonsingular if it is forced to be nonsingular by its pattern of zero, negative, and positive entries. We give structural characterizations of sign-nonsingular matrices, digraphs with no even length dicycles, and square nonnegative real matrices whose permanent and determinant are equal. The structural characterizations, which are topological in nature, imply polynomial algorithms.
\end{abstract}

\section{Introduction}

Pólya's permanent problem can be "traced back to an innocent exercise from 1913." ${ }^{2}$ There are many equivalent versions, such as characterizing when $\operatorname{det}(B)=\operatorname{perm}(B)$ for a square nonnegative real matrix $B$, when all dicycles of a digraph have odd length, or when a square real matrix is sign-nonsingular.

In this section we start with some basic definitions, concepts, and theorems. Then we briefly illustrate why the versions of the previous paragraph are equivalent. Following this we state the Main Theorem which solves all versions of Pólya's permanent problem. Finally, we outline the contents of the rest of the paper.

There are some nonstandard figure conventions used in this paper. The notation "(F. $N)$ " appears in the text when Figure number $N$ is relevant. There are variations such as "(F. Ni, p. $x)$ ", where part $i$ of Figure $N$ on page $x$ is relevant. If part $i$ of Figure $N$ is a graph, we refer to it as $H_{N i}$. Further figure conventions are explained in the second paragraphs of Sections 6 and 8 .

We assume the reader is familiar with elementary linear algebra and complexity theory. For an informal discussion of complexity theory see Lovász and Plummer [28] or Plummer [35]. We will use the graph terminology of Bondy and Murty [3].

*Support from NSERC is gratefully acknowledged.

${ }^{1}$ Quoted from Brualdi and Shader [6]. 
All graphs in this paper are simple, that is, they do not have loops or multiple edges. Let $G$ be a graph. We denote the vertex set by $V(G)$, the number of vertices by $\nu(G)$, the edge set by $E(G)$, and the number of edges by $\varepsilon(G)$. If $s$ is a vertex or edge of $G$, then we say $G$ uses $s$, and $s$ is on $G$.

The degree of a vertex $v$ is the number of edges incident with $v$. The minimum degree $\delta$ (respectively, maximum degree $\Delta$ ) of $G$ is the smallest (respectively, largest) degree of one of its vertices. If $S \subset V(G)$, then $N(S)$ is the set of all vertices in $V(G)-S$ which are adjacent to a vertex in $S$. If $H$ is a subgraph of $G$, and $e$ is an edge of $G$ incident with exactly one vertex on $H$, then we say $e$ is incident with $H$.

An $n$-cycle is a cycle with $n$ vertices. Graphs are disjoint if their vertex sets are disjoint. The sum of graphs $G_{1}, \ldots, G_{n}$ is the graph with vertex set $\cup_{i=1}^{n} V\left(G_{i}\right)$ and edge set $\cup_{i=1}^{n} E\left(G_{i}\right)$, and it is denoted by $G_{1}+\cdots+G_{n}$ and $\sum_{i=1}^{n} G_{i}$. The intersection of graphs $G_{1}$ and $G_{2}$ is the graph with vertex set $V\left(G_{1}\right) \cap V\left(G_{2}\right)$ and edge set $E\left(G_{1}\right) \cap E\left(G_{2}\right)$, and it is denoted by $G_{1} \cap G_{2}$.

If $G$ has a subgraph isomorphic to a graph $K$, then we say $G$ has a $K$ (subgraph). If $G$ is isomorphic to $K$, then we say $G$ is a $K$. A subgraph $H$ of $G$ is proper if $H \neq G$ and $H$ is not the empty graph.

The origin and terminus of a path are called its ends. A vertex on a path is an intermediate vertex if it is not an end. Two or more paths are internally disjoint if every vertex on two of the paths is an end of both. Suppose $G$ has minimum degree at least two, and $P$ is a path of $G$. We say $P$ is a 2-path if its ends have degree at least three, and all its intermediate vertices have degree two. If $G$ has only one 2-path with ends $a$ and $b$, then we denote it by $P_{a b}$.

If $X \subset V(G)$, then $G[X]$ is the subgraph of $G$ with vertex set $X$ whose edge set consists of the edges of $G$ having both ends in $X$. If $F \subset E(G)$, then $G[F]$ is the subgraph with edge set $F$ whose vertex set consists of the vertices incident with edges in $F$. If $S \subset V(G) \cup E(G)$, then $G-S$ is the graph obtained from $G$ by removing all vertices and edges in $S$ (and the edges incident with vertices in $S$ ). If $s$ is a vertex or edge of $G$, then $G-s$ is $G-\{s\}$. If $P$ is a path of $G$, then $G-P$ is the graph obtain from $G$ by removing all edges and intermediate vertices of $P$. (The ends of $P$ stay.) If $P_{1}$ and $P_{2}$ are internally disjoint paths, then $G-\left(P_{1}+P_{2}\right)$ is defined to be $\left(G-P_{1}\right)-P_{2}$.

Let $P=v_{1} v_{2} \cdots v_{n}$ be a path. If $1 \leq i \leq j \leq n$, then the subpath $v_{i} v_{i+1} \cdots v_{j}$ is denoted by $P\left[v_{i}, v_{j}\right]$. The subpath $P\left[v_{i}, v_{j}\right]-v_{i}$ is denoted by $P\left(v_{i}, v_{j}\right]$, and the subpath $P\left[v_{i}, v_{j}\right]-\left\{v_{i}, v_{j}\right\}$ is denoted by $P\left(v_{i}, v_{j}\right)$. If $1 \leq i_{1} \leq i_{2} \leq \cdots \leq i_{k} \leq n$, then we say that we have the order $v_{i_{1}}, v_{i_{2}}, \ldots, v_{i_{k}}$ on $P$. Suppose $x_{1}, x_{2}, \ldots, x_{k}$ are vertices on a cycle $C$. We say that we have the cyclic order $x_{1}, x_{2}, \ldots, x_{k}$ on $C$ if we have the order $x_{1}, x_{2}, \ldots, x_{k}$ on the path $C-e$ for some edge $e$ of $C$.

A matching of $G$ is a set of pairwise nonadjacent edges of $G$. We say a matching saturates a vertex $x$ if some edge in the matching is incident with $x$. A matching is perfect if it saturates all vertices of $G$. If $M$ is a perfect matching, then a cycle $C$ is $M$-alternating if $M \cap E(C)$ is a perfect matching of $C$. The symmetric difference $M \triangle E(C)$ is also a perfect matching, and we say it is obtained by shifting $M$ on $C$. An alternating cycle is a cycle which is $M$-alternating for some perfect matching $M$. 

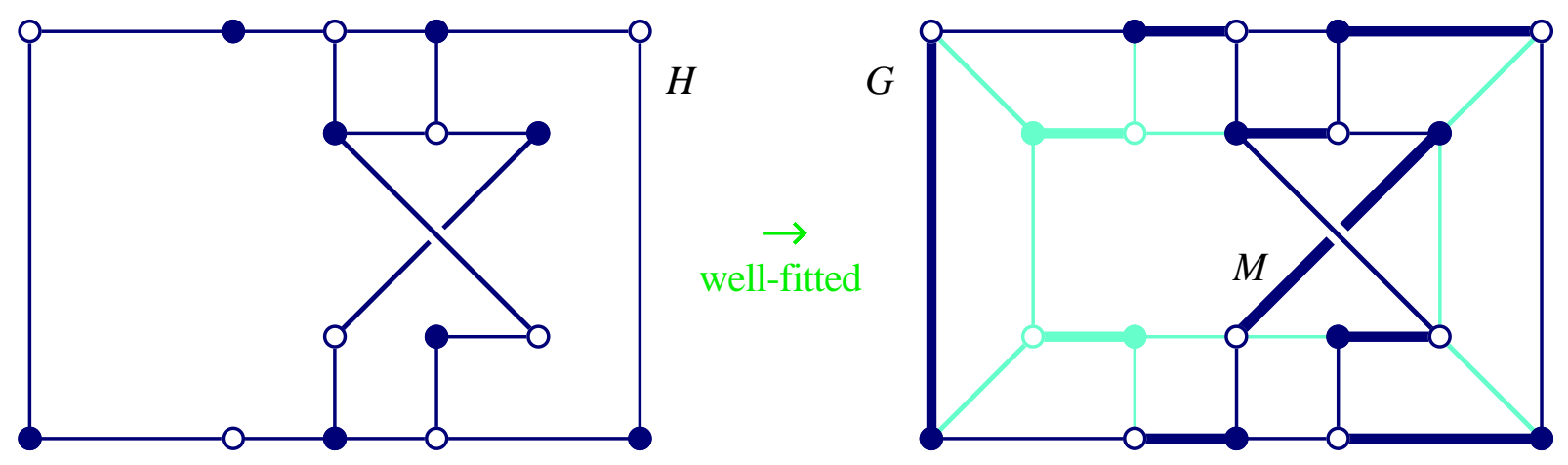

Figure 1: A bipartite graph $G$ with a well-fitted $K_{3,3}$ bisubdivision $H$.

If $H$ is obtained from graph $K$ by replacing some of its edges by paths of odd length (or equivalently, subdividing some of its edges an even number of times), then we say $H$ is a bisubdivision of $K$, or a $K$ bisubdivision ${ }^{2}$ (F. 1). Note that a bisubdivision of a bipartite graph is also bipartite.

The next concept is a generalization of alternating cycles. Suppose $H$ is a subgraph, and $M$ is a perfect matching of $G$ (F.1). We say $H$ is well-fitted to $M$ if for every edge $e$ in $M$, either $e$ is on $H$, or $e$ has no end on $H$. A subgraph $H$ is well-fitted to $G$ if $H$ is well-fitted to some perfect matching of $G$. We also say $G$ has a well-fitted $H$.

All digraphs (directed graphs) in this paper are strict, that is, they do not have loops or parallel arcs. Let $D$ be a digraph. We denote the vertex set by $V(D)$ and the arc set by $A(D)$. By dicycle and dipath we mean directed cycle and directed path, respectively. The origin and terminus of a dipath are called its ends. If $X$ and $Y$ are disjoint subsets of $V(D)$, then a dipath from $X$ to $Y$ is a dipath having origin in $X$, terminus in $Y$, and no intermediate vertices in $X \cup Y$. If $x$ and $y$ are vertices of $D$, then an $(x, y)$-dipath is a dipath from $\{x\}$ to $\{y\}$. Digraphs are disjoint if their vertex sets are disjoint.

Let $\mathcal{S}$ be a subset of the real numbers. An $\mathcal{S}$-edge weighting $w$ of a graph $G$ is a function $w: E(G) \rightarrow \mathcal{S}$. An $\mathcal{S}$-arc weighting $\vec{w}$ of a digraph $D$ is a function $\vec{w}: A(D) \rightarrow \mathcal{S}$.

Let $G$ be a bipartite graph with a $\{-1,1\}$-edge weighting $w$ (F. 2, p. 6, graph $G$ ). Suppose $C$ is a cycle. Let $w(C)=\prod_{e \in E(C)} w(e)$. We say $C$ is $w$-unbalanced if

$$
w(C)=\left\{\begin{array}{cll}
-1 & \text { if } \nu(C) \equiv 0 & (\bmod 4), \\
1 & \text { if } \nu(C) \equiv 2 & (\bmod 4) .
\end{array}\right.
$$

If $w$ is implicit we simply say $C$ is unbalanced. We say $w$ is unbalanced if $G$ has a perfect matching and every alternating cycle (with respect to any perfect matching) is unbalanced. A cycle is balanced if it is not unbalanced. A cycle whose edges are alternately weighted -1 and 1 is an example of a balanced cycle.

\footnotetext{
${ }^{2}$ In the literature $H$ is referred to as both an even $K$ and an odd $K$, which is why we choose to call $H$ a $K$ bisubdivision.
} 
Let $D$ be a digraph with a $\{-1,1\}$-arc weighting $\vec{w}$ (F. 2, p. 6, digraph $D$ ). Suppose $C$ is a dicycle of $D$. Let $\vec{w}(C)=\prod_{e \in A(C)} \vec{w}(e) . C$ is $\vec{w}$-negative if $\vec{w}(C)=-1$ and $\vec{w}$-positive if $\vec{w}(C)=1$. We say $\vec{w}$ is negative if every dicycle of $D$ is $\vec{w}$-negative.

Let $w$ be a $\{-1,1\}$-edge weighting of a bipartite graph $G$. If we replace $w(e)$ by its negative for every edge $e$ incident with a vertex $v$ of $G$, then we say the resulting $\{-1,1\}$-edge weighting is obtained from $w$ by switching at $v$. If $w^{\prime}$ is obtained from $w$ by sequentially switching at vertices $v_{1}, \ldots, v_{k}$, then we say $w$ and $w^{\prime}$ are equivalent. Note that $w^{\prime}$ is invariant under changing the order of $v_{1}, \ldots, v_{k}$. Similar definitions hold for $\{-1,1\}$-arc weightings of digraphs.

Let $\mathcal{S}$ be a set. An $\mathcal{S}$-matrix is a matrix with all its entries in $\mathcal{S}$. Let $A=\left[a_{i j}\right]$ be an $m \times n$ real matrix. The support of $A$ is $\left\{(i, j) \mid a_{i j} \neq 0\right\}$. The sign pattern of $A$ is the $m \times n\{-, 0,+\}$-matrix $S=\left[s_{i j}\right]$ (p. 12, matrices $A$ and $S$ ) such that

$$
s_{i j}=\left\{\begin{aligned}
- & \text { if } a_{i j}<0, \\
0 & \text { if } a_{i j}=0, \text { and } \\
+ & \text { if } a_{i j}>0
\end{aligned}\right.
$$

for $i=1, \ldots, m$ and $j=1, \ldots, n$. If $A$ is a square matrix, then $A$ is sign-nonsingular if every matrix with the same sign pattern as $A$ is nonsingular (F. 2, matrix $A$ ).

Suppose $A=\left[a_{i j}\right]$ is an $m \times n$ real matrix (F. 2, $A$ and $G$ ). Let $G$ be the bipartite graph with colour classes $\mathcal{R}=\left\{r_{1}, \ldots, r_{m}\right\}$ and $\mathcal{C}=\left\{c_{1}, \ldots, c_{n}\right\}$ such that $r_{i} c_{j}$ is an edge of $G$ if and only if $a_{i j} \neq 0$ for $i=1, \ldots, m$ and $j=1, \ldots, n$. We say $G$ and $A$ correspond, and $G$ is the bipartite graph of $A$, where $\mathcal{R}$ is the set of row vertices and $\mathcal{C}$ is the set of column vertices. In defining $G$ we are implicitly ordering its set $\{\mathcal{R}, \mathcal{C}\}$ of colour classes, and each of its colour classes. Note that $G$ is isomorphic to the bipartite graph of any matrix obtained from $A$ by permuting its rows, permuting its columns, or taking its transpose. Let $w$ be the $\{-1,1\}$-edge weighting of $G$ such that

$$
w\left(r_{i} c_{j}\right)=\left\{\begin{array}{cl}
-1 & \text { if } a_{i j}<0, \text { and } \\
1 & \text { if } a_{i j}>0,
\end{array}\right.
$$

for every edge $r_{i} c_{j}$ of $G$. We say $(G, w)$ is the weighted bipartite graph of $A$, and $(G, w)$ and $A$ correspond.

Suppose $D$ is a digraph with vertex set $\left\{v_{1}, \ldots, v_{n}\right\}$ (F. 2, D and $G$ ). Let $G$ be the bipartite graph with colour classes $\left\{r_{1}, \ldots, r_{n}\right\}$ and $\left\{c_{1}, \ldots, c_{n}\right\}$ such that $r_{1} c_{1}, \ldots, r_{n} c_{n}$ are edges of $G$, and $r_{i} c_{j}$ is an edge of $G$ if and only if $v_{i} v_{j}$ is an arc of $D$, for every $i$ and $j$ in $\{1, \ldots, n\}$ such that $i \neq j$. We say $G$ is the bipartite graph of $D$, and $G$ and $D$ correspond. We also say that the perfect matching $\left\{r_{1} c_{1}, \ldots, r_{n} c_{n}\right\}$ of $G$ corresponds to the vertex set of $D$, and that $r_{i} c_{i}$ corresponds to $v_{i}$ for $i=1, \ldots, n$. Note that for every perfect matching $M$ of $G$, there is a digraph $D^{\prime}$ corresponding to $G$ such that $M$ corresponds to the vertex set of $D^{\prime}$. Suppose $\vec{w}$ is a $\{-1,1\}$-arc weighting of $D$. Let $w$ be the $\{-1,1\}$-edge weighting of $G$ such that $w\left(r_{i} c_{i}\right)=-1$ for $i=1, \ldots, n$, and $w\left(r_{i} c_{j}\right)=\vec{w}\left(v_{i} v_{j}\right)$ for every edge $r_{i} c_{j}$ of $G$ such that $i \neq j$. We say $(G, w)$ is the weighted bipartite graph of $(D, \vec{w})$, and $(G, w)$ and $(D, \vec{w})$ correspond. 
A graph $G$ is connected if there is an $(x, y)$-path for every pair of vertices $x$ and $y$. A component of $G$ is a maximal connected subgraph. Suppose $k \geq 1$. A $k$-vertex cut of $G$ is a set $X$ of $k$ vertices such that $G-X$ is not connected. $G$ is $n$-connected if $\nu(G) \geq n+1$ and $G$ does not have an $(n-1)$-vertex cut.

A digraph $D$ is strongly connected if there is an $(x, y)$-dipath for every ordered pair of vertices $(x, y)$. A strong component of $D$ is a maximal strongly connected subdigraph. A $k$-vertex cut of $D$ is a set $X$ of $k$ vertices such that $D-X$ is not strongly connected. $D$ is strongly $n$-connected if $\nu(D) \geq n+1$ and $D$ does not have an $(n-1)$-vertex cut.

A graph $G$ is $k$-extendible if

- $\nu \geq 2 k+2$,

- $G$ is connected,

- $G$ has a matching of size $k$, and

- for every matching $M_{k}$ of size $k$, there is a perfect matching containing $M_{k}$.

The fourth condition is the most important. The first three are included so that graphs such as stars are excluded, and so that Theorem 3 is true. A brace is a 2-extendible bipartite graph.

Next we state three classical results which are fundamental to the work of this paper.

Theorem 1 (Menger [32]) Let $D$ be a strongly k-connected digraph. Suppose $X$ and $Y$ are disjoint nonempty subsets of $V(D)$. Then there exist $k$ internally disjoint dipaths $P_{1}, \ldots, P_{k}$ from $X$ to $Y$. If $|X| \geq k$ (respectively, $|Y| \geq k$ ), then $P_{1}, \ldots, P_{k}$ can be chosen to have distinct origins (respectively, termini).

Theorem 2 (Frobenius [8, 9] and König [19, 20]) A bipartite graph $G$ has a matching saturating all vertices in a colour class $A$ if and only if $|X| \leq|N(X)|$ for every subset $X$ of $A$.

In the next theorem, the equivalence of statements (a) and (b) when $k=1$ is due to Hetyei [13]. Brualdi and Perfect [4] proved a matrix version of the equivalence of statements (a) and (b) for all $k$. The equivalence of statements (a), (c), and (d) for $k=1,2$ is stated in Brualdi and Shader [6, pages 42 and 124]. In particular, braces correspond to strongly 2-connected digraphs.

Theorem 3 Let $G$ be a bipartite graph with a perfect matching, and let $A$ be a colour class of $G$. If $k \geq 1$, then the following statements are equivalent.

a) $G$ is k-extendible.

b) $G$ is connected, $k+1 \leq|A|$, and for every subset $X$ of $A$ such that $1 \leq|X| \leq|A|-k$, we have $|X|+k \leq|N(X)|$.

c) Some digraph corresponding to $G$ is strongly $k$-connected.

d) All digraphs corresponding to $G$ are strongly $k$-connected. 
$A$ is sign-nonsingular.

$$
\begin{gathered}
A=\left[\begin{array}{ccc}
-1 & -1 & 1 \\
1 & -1 & 0 \\
-1 & -1 & -1
\end{array}\right] \quad \longleftrightarrow \\
B=\left[\begin{array}{lllll}
1 & 1 & 0 & 1 & 0 \\
0 & 1 & 0 & 0 & 1 \\
1 & 1 & 1 & 0 & 0 \\
0 & 0 & 1 & 1 & 0 \\
1 & 0 & 0 & 0 & 1
\end{array}\right] \longleftrightarrow \\
\operatorname{perm}(B)=4=\operatorname{det}(B)
\end{gathered}
$$
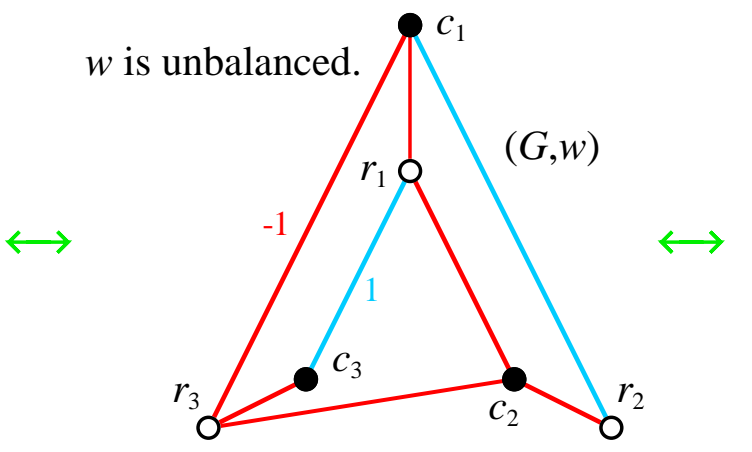

$\vec{w}$ is negative.
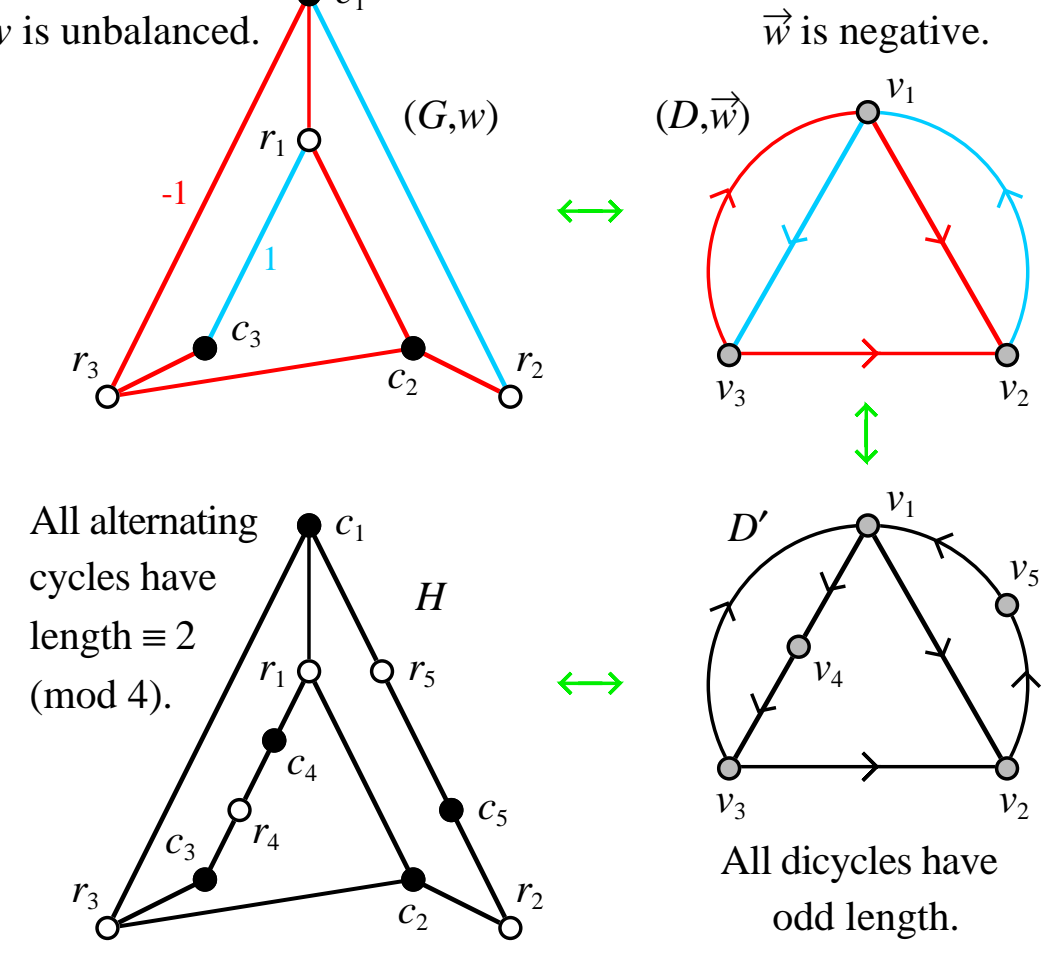

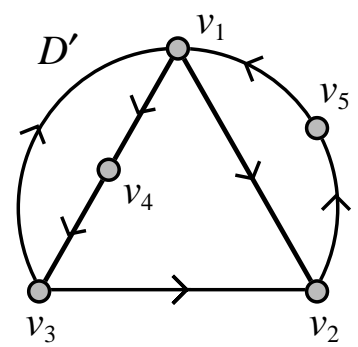

All dicycles have odd length.

Figure 2: Versions of Pólya's permanent problem.

In section 4 we will state many versions of Pólya's permanent problem and prove they are all equivalent. For now we will only give examples to informally show the equivalence of the three versions given in the first paragraph of this introduction. In Figure 2, we see $(G, w)$ corresponds to both $A$ and $(D, \vec{w})$, and $H$ corresponds to both $B$ and $D^{\prime}$. As well, $D$ naturally corresponds to $D^{\prime}$ : arcs of $D$ with weight 1 are replaced by dipaths of length 2 to give $D^{\prime}$. Notice that $A$ is sign-nonsingular, all dicycles of $D^{\prime}$ have odd length, and $\operatorname{det}(B)=\operatorname{perm}(B)$. This concurrence happens in general. Consequently, we have three equivalent problems: characterize sign-nonsingular matrices, characterize digraphs having only odd length dicycles, and characterize nonnegative matrices whose permanent and determinant are equal.

Next we introduce two new versions. "Our goal is to obtain a formulation of the problem which is more convenient for the purpose of solving it." 3 In Figure 2, notice that $w$ is an unbalanced weighting of $G$. Determining when a $\{-1,1\}$-edge weighting of a bipartite graph is unbalanced is another version of the problem. This problem in turn is equivalent to the slightly different problem of determining when a bipartite graph has an unbalanced $\{-1,1\}$-edge weighting. (In one version we are given a graph and a weighting, whereas, in the other version we are only given a graph.) This is the formulation which is solved by the Main Theorem.

\footnotetext{
${ }^{3}$ Quoted from Little [25].
} 

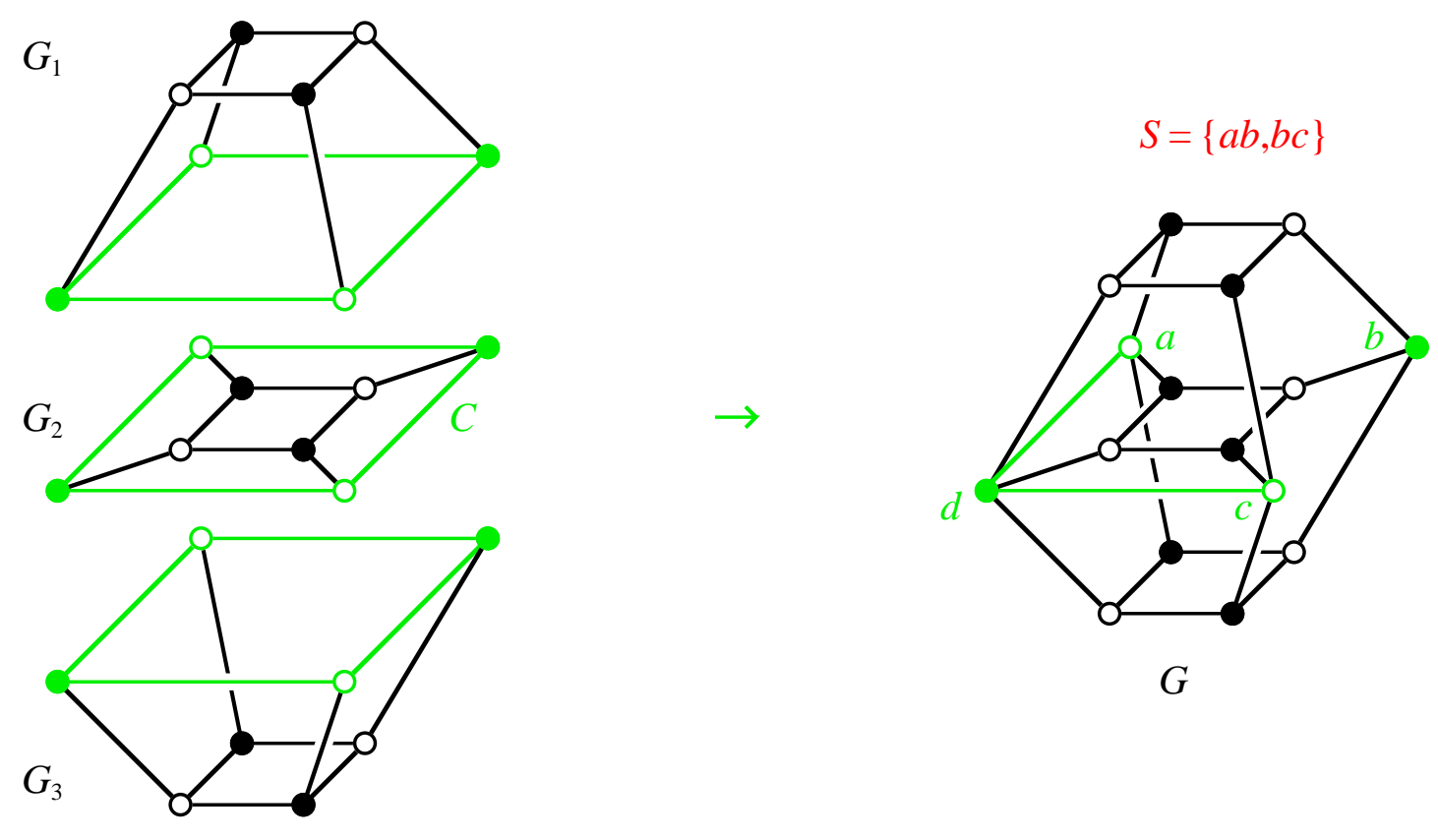

Figure 3: A 4-cycle sum.

In order to state the Main Theorem, we need to define a set $\mathcal{G}$ of graphs (F. 3). Suppose $G_{1}, \ldots, G_{n}$ are bipartite graphs, where $n \geq 3$, such that their pairwise intersection is a 4-cycle $C=$ abcda. Let $S$ be a (not necessarily proper) subset of $E(C)$. The graph $\left(\sum_{i=1}^{n} G_{i}\right)-S$ is called a 4 -cycle sum of $G_{1}, \ldots, G_{n}$ at $C$. (4-cycle sums are closely related to the 2-joins of Brualdi and Shader [6, p. 120].) Let $\mathcal{G}$ consist of

- the Heawood graph $H_{14}$ (F. 4, p. 8),

- all planar braces, and

- all graphs generated from planar braces using 4-cycle sums.

Note that we are allowed to use the 4-cycle sum operation more than once. For example, we obtain another graph in $\mathcal{G}$ if we take five disjoint copies of the graph $G$ of Figure 3 , select a 4-cycle from each copy, and then identify the 4-cycles.

The restriction $n \geq 3$ in the definition of a 4-cycle sum requires some explanation. There are three reasons we do not allow the graph $G$ to be a 4-cycle sum of only two braces $G_{1}$ and $G_{2}$. First, $G$ may not be a brace. Second, using such 4-cycle sums in the definition of $\mathcal{G}$ would not add any new braces to $\mathcal{G}$. For example, if $G_{1}$ and $G_{2}$ are planar, then $G$ is also planar; and so $G$ is already in $\mathcal{G}$ if it is a brace. Third, inductive proofs involving $\mathcal{G}$ would be more difficult.

Main Theorem. The following statements are equivalent for a bipartite graph $G$.

1) $G$ is a brace which has an unbalanced $\{-1,1\}$-edge weighting.

2) $G$ is a brace which does not have a well-fitted $K_{3,3}$ bisubdivision.

3) $G$ is in $\mathcal{G}$. 
Let $\mathcal{U}$ be the class of bipartite graphs which have unbalanced $\{-1,1\}$-edge weightings. In section 4 we show that the problem of characterizing $\mathcal{U}$ can be reduced to characterizing the braces in $\mathcal{U}$. The Main Theorem gives a good characterization of these braces, that is, it shows that determining if a given brace is in $\mathcal{U}$ is an algorithmic question in NP^co-NP. To show a brace is in $\mathcal{U}$, we show it is in $\mathcal{G}$. To show a brace is not in $\mathcal{U}$, we show it has a well-fitted $K_{3,3}$ bisubdivision. A polynomial algorithm for determining if a given bipartite graph is in $\mathcal{U}$ can be derived (with a lot of work) from the Main Theorem and its proof. A full discussion about an algorithm is found in Robertson, Seymour, and Thomas [37].

Figure 4 shows four naturally corresponding objects from different versions of Pólya's permanent problem. They play an important role, as we see from the Main Theorem. They were first connected to the problem by Tinsley [47]. The digraph $D_{7}$ was found independently by Boyd (communicated to Thomassen [45, page 40]), Koh [18], and Seymour [41]. It is the only strongly 2-connected digraph with no even dicycle [30].

Section 2 outlines the proof of the Main Theorem. Sections 3 and 4 are about the history and versions of Pólya's permanent problem. Sections 5 to 9 contain the proof of the Main Theorem. Section 10 relates the Main Theorem to results of Little, Seymour, and Thomassen.

$$
\begin{aligned}
T & =\left[\begin{array}{lllllll}
1 & 1 & 0 & 0 & 0 & 1 & 0 \\
0 & 1 & 1 & 0 & 0 & 0 & 1 \\
1 & 0 & 1 & 1 & 0 & 0 & 0 \\
0 & 1 & 0 & 1 & 1 & 0 & 0 \\
0 & 0 & 1 & 0 & 1 & 1 & 0 \\
0 & 0 & 0 & 1 & 0 & 1 & 1 \\
1 & 0 & 0 & 0 & 1 & 0 & 1
\end{array}\right] \\
\operatorname{det}(T)=24= & \operatorname{perm}(T)
\end{aligned}
$$

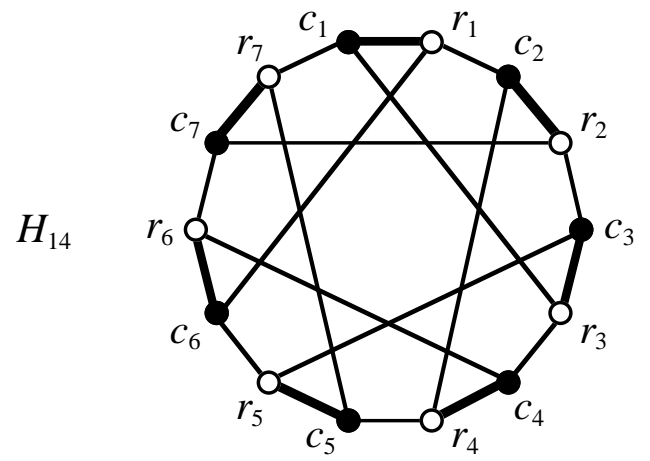

Each alternating cycle has length 6,10 , or 14 .

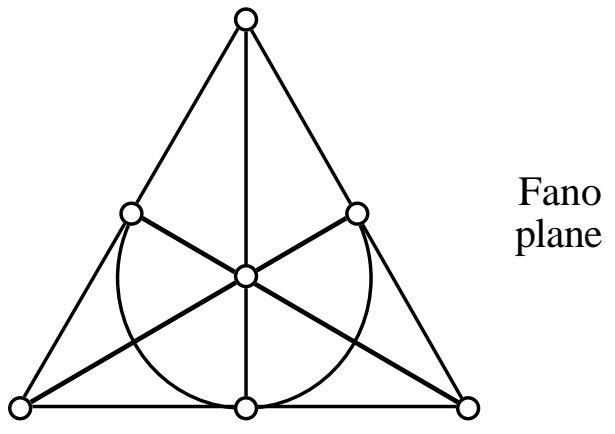

There is no balanced 2-colouring.

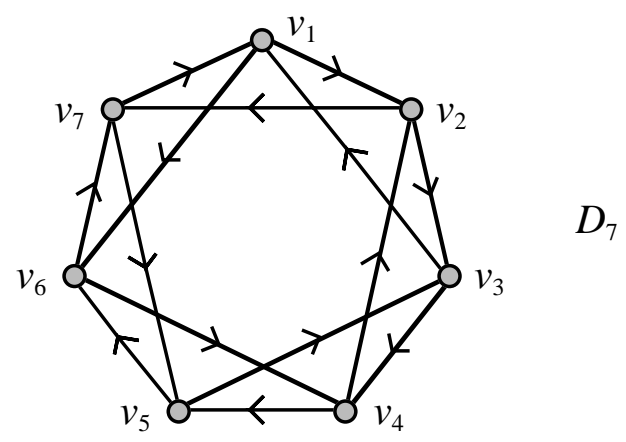

Each dicycle has length 3,5 , or 7 .

Figure 4: The Tinsley, Heawood, Fano, Boyd, Ko, and Seymour collection. 


\section{Proof Outline}

In this section we outline the proof of the Main Theorem. To prove that the three statements of the Main Theorem are equivalent, we show $(1 \Rightarrow 2 \Rightarrow 3 \Rightarrow 1)$.

We prove the relatively easy implication $(1 \Rightarrow 2)$ by showing that if a bipartite graph $G$ has a well-fitted $K_{3,3}$ bisubdivision, then $G$ can not have an unbalanced $\{-1,1\}$-edge weighting.

We prove $(3 \Rightarrow 1)$ using induction. In the induction basis, we show that $H_{14}$ is a brace, and that unbalanced weightings can be constructed for $H_{14}$ and all planar braces. In the induction step, we suppose $G$ is a 4 -cycle sum of $G_{1}, \ldots, G_{n}$. We use unbalanced weightings of $G_{1}, \ldots, G_{n}$ to construct an unbalanced weighting of $G$. Furthermore, we show that if $G_{1}, \ldots, G_{n}$ are braces, then either $G$ is a brace, or $G$ is one exceptional graph $H_{10}$ (which is not in $\mathcal{G}$ ).
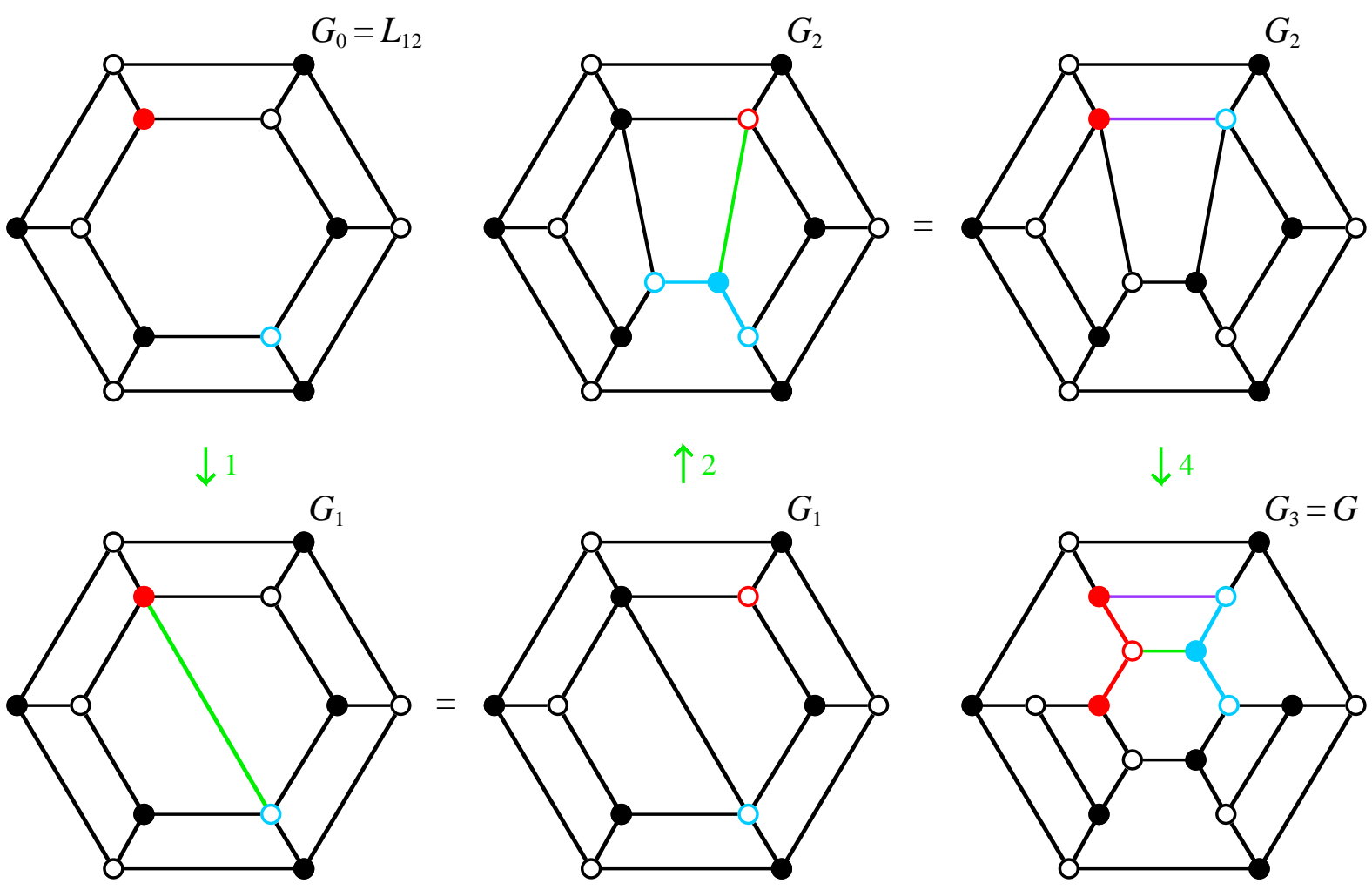

Figure 5: An example for Theorem 24 (p. 39).

The difficult part of the proof is showing $(2 \Rightarrow 3)$. It suffices to show that if $G$ is a brace, then $G \in \mathcal{G}$ or $G$ has a well-fitted $K_{3,3}$ bisubdivision. This is done by induction on $\varepsilon(G)$. Here we need a theorem from [31] which roughly says that all braces can be constructed from a "base set" $\mathcal{B}$ using "local operations". (Section 5 contains a precise statement of the theorem, and Figure 5 gives an example of it in action.) For the induction basis, it is easy to verify that each brace in $\mathcal{B}$ is either in $\mathcal{G}$, or has a well-fitted $K_{3,3}$ bisubdivision. 


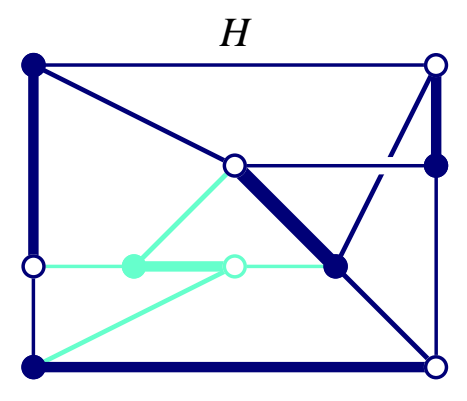

$H$ has a well-fitted $K_{3,3}$ bisubdivision.
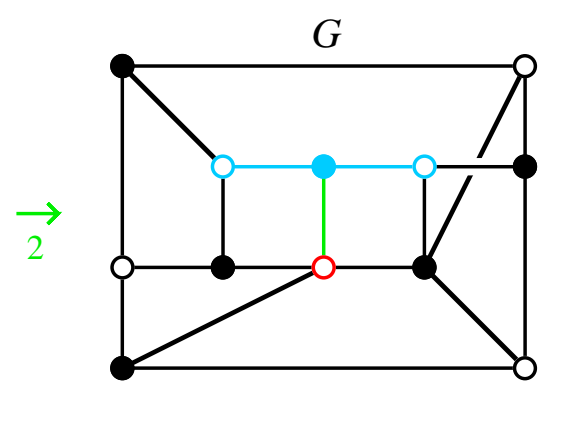

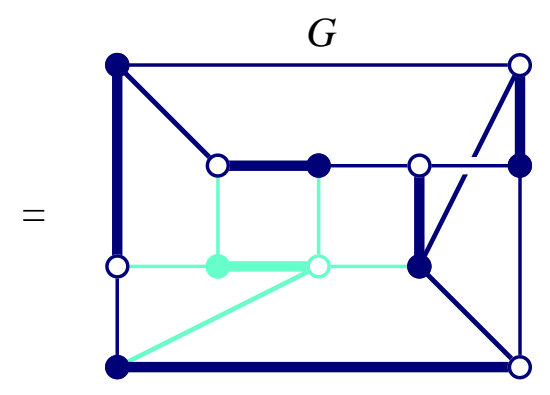

$G$ has a well-fitted $K_{3,3}$ bisubdivision.

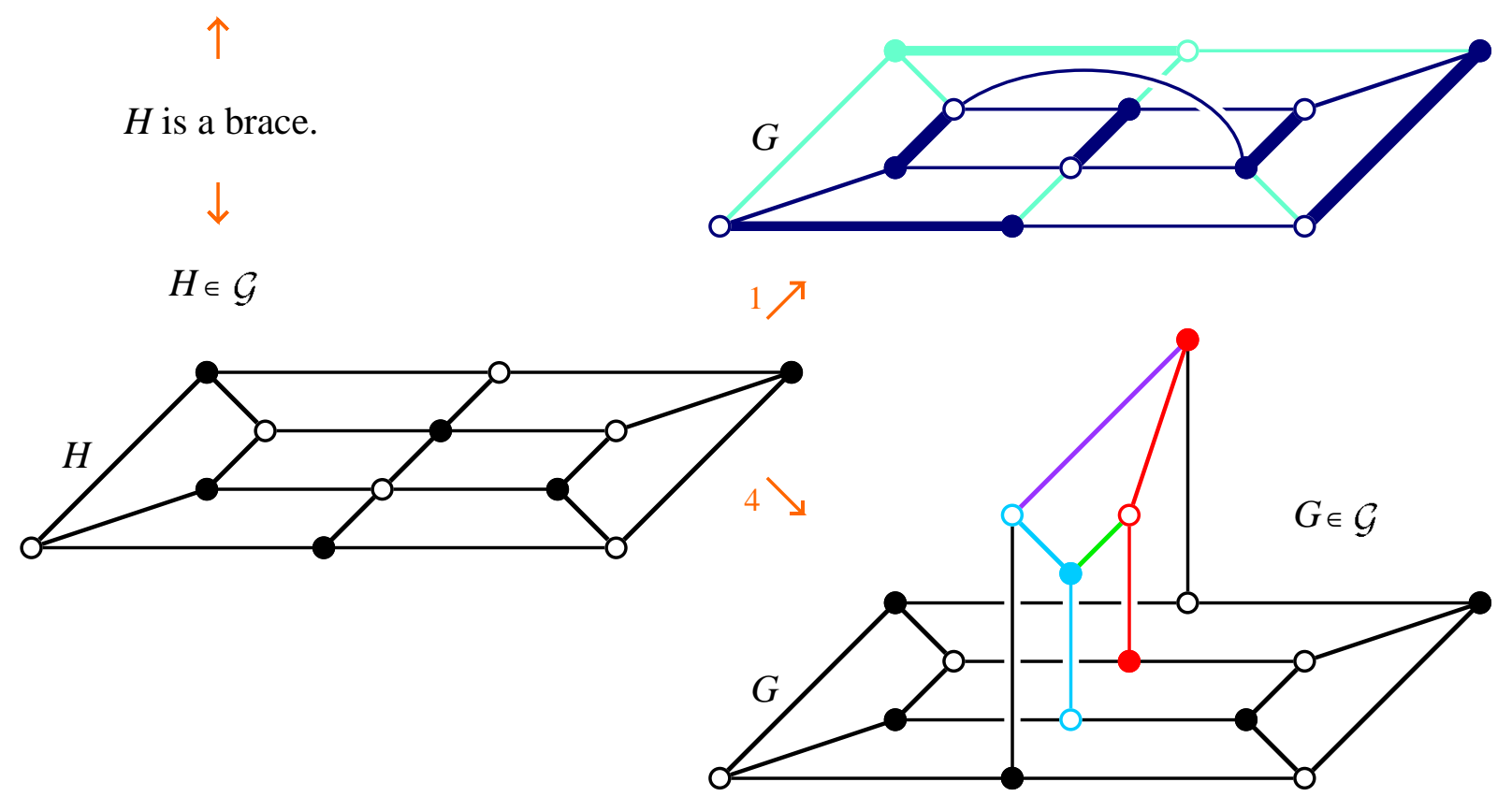

Figure 6: The induction step in the proof of the Main Theorem.

The induction step is illustrated by Figure 6. (Note that the lower right graph is in $\mathcal{G}$ because it is a 4-cycle sum of three planar braces.) If $G \notin \mathcal{B}$, then $G$ can be obtained from a smaller brace $H$ using a local operation. The result then holds for $H$. If $H$ has a well-fitted $K_{3,3}$ bisubdivision, then it is routine to show $G$ does also. Hence, we may assume $H \in \mathcal{G}$. We then show $G$ is also in $\mathcal{G}$, or $G$ has a well-fitted $K_{3,3}$ bisubdivision. This part of the proof is long because there are many cases. There are many cases because there are four local operations, and because $H$ can be either an $H_{14}$, or a planar brace, or a 4-cycle sum of smaller braces. The reason it works is because we know the structure of $H$ - it's in $\mathcal{G}$ - and because $G$ is obtained from $H$ using a local operation.

Sections $5,6,7$, and 8 contain known results and technical lemmas needed to prove the Main Theorem. Section 9 contains the core of its proof. 


\section{Origins}

In this section we discuss four origins of Pólya's permanent problem.

\section{Permanents}

The permanent and determinant of an $n \times n$ matrix $A=\left[a_{i j}\right]$ are given by

$$
\operatorname{perm}(A)=\sum_{\sigma \in S_{n}} \prod_{i=1}^{n} a_{i, \sigma(i)} \quad \text { and } \quad \operatorname{det}(A)=\sum_{\sigma \in S_{n}} \operatorname{sgn}(\sigma) \prod_{i=1}^{n} a_{i, \sigma(i)},
$$

respectively, where $S_{n}$ is the set of all permutations of $\{1, \ldots, n\}$ and

$$
\operatorname{sgn}(\sigma)=\left\{\begin{array}{cl}
-1 & \text { if } \sigma \text { is the product of an odd number of transpositions, and } \\
1 & \text { if } \sigma \text { is the product of an even number of transpositions, }
\end{array}\right.
$$

for every $\sigma$ in $S_{n}$. The terms in the above formulas for perm $(A)$ and $\operatorname{det}(A)$ will be called the terms of perm $(A)$ and $\operatorname{det}(A)$, respectively.

The formula for perm $(A)$ is similar to, and simpler than, the formula for $\operatorname{det}(A)$. But there is a polynomial algorithm for calculating determinants, whereas calculating permanents is \#P-complete ${ }^{4}$ as shown by Valiant [49]. This apparent paradox can be explained by noting that Gaussian elimination, not the given formula, is used to efficiently calculate determinants. Given the similarity between the formulas for the permanent and the determinant, Pólya [36] asked if it was possible to calculate permanents using determinants. Specifically, given a matrix $A=\left[a_{i j}\right]$, was it possible to change the signs of some of the entries of $A$ to give a new matrix $B=\left[b_{i j}\right]$ such that the corresponding terms of perm $(A)$ and $\operatorname{det}(B)$ were equal, that is,

$$
\prod_{i=1}^{n} a_{i, \sigma(i)}=\operatorname{sgn}(\sigma) \prod_{i=1}^{n} b_{i, \sigma(i)}
$$

for every $\sigma$ in $S_{n}$. We would then have perm $(A)=\operatorname{det}(B)$. Here is an example.

$$
\begin{aligned}
& A=\left[\begin{array}{ccc}
1 & 1 & 0 \\
1 & 1 & 1 \\
1 & 1 & 1
\end{array}\right] \quad \begin{array}{c}
\operatorname{perm}(A)=a_{11} a_{22} a_{33}+a_{11} a_{23} a_{32}+a_{12} a_{21} a_{33} \\
+a_{12} a_{23} a_{31}+a_{13} a_{21} a_{32}+a_{13} a_{22} a_{31} \\
=1+1+1+1+0+0=4
\end{array} \\
& B=\left[\begin{array}{rrr}
-1 & 1 & 0 \\
-1 & -1 & 1 \\
1 & 1 & 1
\end{array}\right] \quad \begin{array}{c}
\operatorname{det}(B)=b_{11} b_{22} b_{33}-b_{11} b_{23} b_{32}-b_{12} b_{21} b_{33} \\
+b_{12} b_{23} b_{31}+b_{13} b_{21} b_{32}-b_{13} b_{22} b_{31} \\
=1+1+1+1+0+0=4
\end{array}
\end{aligned}
$$

It was soon discovered by Szegö [43] that for every $n \geq 3$, Pólya's method does not work for some $n \times n$ matrix. This lead to the obvious question of when Pólya's method can be used to calculate the permanent of a matrix, and this is Pólya's permanent problem.

\footnotetext{
${ }^{4}$ A complexity class consisting of hard counting problems. Counting the number of Hamiltonian cycles of a graph is another problem in this class.
} 


\section{Sign-Solvable Sign Systems}

Let $S$ be an $n \times n\{-, 0,+\}$-matrix and $\bar{t}$ be an $n \times 1\{-, 0,+\}$-matrix. $S \bar{x}=\bar{t}$ denotes the set of all matrix equations $A \bar{x}=\bar{b}$ such that $A$ has sign pattern $S$, and $\bar{b}$ has sign pattern $\bar{t}$. We say $S \bar{x}=\bar{t}$ is a sign system, and the matrix equations in $S \bar{x}=\bar{t}$ are its instances. $S \bar{x}=\bar{t}$ is sign-solvable if all instances have a unique solution and all the solutions of the instances have the same sign-pattern.

$$
\begin{gathered}
A \bar{x}=\left[\begin{array}{cc}
3 & -2 \\
-1 & -1
\end{array}\right]\left[\begin{array}{l}
x \\
y
\end{array}\right]=\left[\begin{array}{c}
0 \\
-5
\end{array}\right]=\bar{b} \Rightarrow \bar{x}=\left[\begin{array}{l}
x \\
y
\end{array}\right]=\left[\begin{array}{l}
2 \\
3
\end{array}\right] \\
S \bar{x}=\left[\begin{array}{ll}
+ & - \\
- & -
\end{array}\right]\left[\begin{array}{l}
x \\
y
\end{array}\right]=\left[\begin{array}{c}
0 \\
-
\end{array}\right]=\bar{t} \Rightarrow \bar{x}=\left[\begin{array}{l}
x \\
y
\end{array}\right]=\left[\begin{array}{l}
+ \\
+
\end{array}\right]
\end{gathered}
$$

For the sign-system of the example, given an arbitrary instance

$$
\left[\begin{array}{cc}
a & -b \\
-c & -d
\end{array}\right]\left[\begin{array}{l}
x \\
y
\end{array}\right]=\left[\begin{array}{c}
0 \\
-e
\end{array}\right], \text { where } a, b, c, d, \text { and } e \text { are positive numbers, }
$$

we can use Cramer's Rule to find the solution as follows.

$$
x=\frac{\left|\begin{array}{cc}
0 & -b \\
-e & -d
\end{array}\right|}{\left|\begin{array}{cc}
a & -b \\
-c & -d
\end{array}\right|}=\frac{-b e}{-a d-b c}>0 \quad y=\frac{\left|\begin{array}{cc}
a & 0 \\
-c & -e
\end{array}\right|}{\left|\begin{array}{cc}
a & -b \\
-c & -d
\end{array}\right|}=\frac{-a e}{-a d-b c}>0
$$

Note that all the determinants are negative for all choices of $a, b, c, d$, and $e$. Consequently, every instance has a unique solution, and both entries of the solution are positive. Thus, the sign system is sign-solvable. All these negative determinants are due to the fact that the matrices in question are sign-nonsingular. (See Theorem 4 on page 16.)

It has been shown that the last ingredient needed to characterize sign-solvable sign systems is a characterization of sign-nonsingular matrices. (See Brualdi and Shader [6], or Klee, Ladner, and Manber [17].) Determining if a matrix is sign-nonsingular is a version of Pólya's permanent problem, as we will see in the next section.

The possibility of studying sign-solvable sign systems was first raised by Samuelson [38] in his book Foundations of Economic Analysis (first edition, 1947). Given two economic variables, we might not know exactly how one affects the other. But we may know a qualitative relationship, that is, one causes the other to rise, or one causes the other to fall, or they are independent of one another. Samuelson asked the following general question: given some qualitative relationships between variables, what other qualitative relationships can be derived? Samuelson's question and the papers of Gorman [11] and Lancaster $[\mathbf{2 1}, \mathbf{2 2}, \mathbf{2 3}]$ started the study of sign-solvability. 
Samuelson gave the following example, which is also in Brualdi and Shader $[\mathbf{6}, \mathbf{p .} \mathbf{1}]$. We have five parameters from the coffee trade.

- $t$ is a measure of people's taste for coffee.

- $p(t)$ is the price per bag, as a function of $t$.

- $V(t)$ is the volume, that is, the number of bags sold per day, as a function of $t$.

- $S(p)$ is the supply in bags per day, as a function of $p$.

- $D(p, t)$ is the demand in bags per day, as a function of $p$ and $t$.

Suppose we make three qualitative assumptions.

- $\frac{\partial S}{\partial p}>0$, that is, the supply of coffee increases as the price increases.

- $\frac{\partial D}{\partial p}<0$, that is, the demand for coffee decreases as the price increases.

- $\frac{\partial D}{\partial t}>0$, that is, the demand increases as people's taste for coffee increases.

We will show these assumptions imply $\frac{\partial p}{\partial t}>0$ and $\frac{\partial v}{\partial t}>0$, that is, the price $p$ and the volume $v$ increase as people's taste $t$ for coffee increases.

The volume is always less than or equal to the supply and the demand, that is, $V(t) \leq S(p)$ and $V(t) \leq D(p, t)$. Suppose the volume is (strictly) less than the supply. Then the price will drop, causing $D(p, t)$ to rise by assumption; and so the volume will also be less than the demand. Now with the volume less than both the supply and demand, the volume will increase. Similarly, we can show the volume increases when it is less than the demand. The volume stops increasing, and the market reaches equilibrium, when the supply, demand, and volume are all equal, that is, when

$$
S(p)=V(t)=D(p, t)
$$

Taking partial derivatives with respect to $t$ we obtain

$$
\frac{\partial S}{\partial p} \frac{\partial p}{\partial t}=\frac{\partial V}{\partial t}=\frac{\partial D}{\partial p} \frac{\partial p}{\partial t}+\frac{\partial D}{\partial t} .
$$

Equivalently,

$$
\left[\begin{array}{cc}
\frac{\partial S}{\partial p} & -1 \\
\frac{\partial D}{\partial p} & -1
\end{array}\right]\left[\begin{array}{c}
\frac{\partial p}{\partial t} \\
\frac{\partial V}{\partial t}
\end{array}\right]=\left[\begin{array}{c}
0 \\
-\frac{\partial D}{\partial t}
\end{array}\right]
$$

By our assumptions $\frac{\partial S}{\partial p}>0, \frac{\partial D}{\partial p}<0$, and $\frac{\partial D}{\partial t}>0$, we have an instance of the sign system

$$
\left[\begin{array}{cc}
+ & - \\
- & -
\end{array}\right]\left[\begin{array}{c}
\frac{\partial p}{\partial t} \\
\frac{\partial V}{\partial t}
\end{array}\right]=\left[\begin{array}{c}
0 \\
-
\end{array}\right] .
$$

On the previous page we showed that it is sign-solvable, and that for every instance, both entries of the solution are positive. Hence, the price and the volume increase as people's taste for coffee increases. 


\section{Pfaffian Orientations}

If we replace each edge $x y$ of a graph $G$ by either the $\operatorname{arc} x y$ or the $\operatorname{arc} y x$, then the resulting digraph $\vec{G}$ is called an orientation of $G$. The orientation $\vec{G}$ is Pfaffian if $G$ has at least one perfect matching, and every alternating cycle $C$ of $G$ has an odd number of arcs going in the one direction around $C$ and an odd number of arcs going in the other direction (F. 18, p. 33). In the next section we will see that determining which bipartite graphs have Pfaffian orientations is a version of Pólya's permanent problem. Note that some nonbipartite graphs also have Pfaffian orientations (F. 19, p. 35).

Pfaffian orientations were first examined by Kasteleyn $[\mathbf{1 4}, \mathbf{1 5}, \mathbf{1 6}]$, Fisher [7], and Temperley and Fisher [44]. They were interested in applications in chemistry, such as the dimer problem. (See Lovász and Plummer [28, Section 8.3].) We give another example (F. 7, p. 15).

A single bond in a molecule consists of two electrons shared by two atoms, and a double bond consists of four electrons shared by two atoms. Both hydrocarbons in the example have 16 carbon-carbon single bonds and 10 carbon-hydrogen single bonds. These hydrocarbons are called aromatic because each also has a $\pi$-bond consisting of 14 electrons shared by the 14 carbon atoms. Each hydrocarbon has an associated graph having a vertex for every carbon atom, and an edge for every carbon-carbon single bond. A resonance form of either aromatic hydrocarbon is defined as follows. Choose a perfect matching of the associated graph. For each of the 7 corresponding single carbon-carbon bonds, add two electrons from the $\pi$-bond to obtain a carbon-carbon double bond. (In other words, a $\pi$-bond and 7 "independent" single bonds are replaced by 7 "independent" double bonds.) An aromatic hydrocarbon is said to be a hybrid of its resonance forms. We should note that resonance forms are theoretical molecules, that is, they can not be found in nature. We are describing something that exists as a hybrid of things that do not exist, or as John D. Roberts [33, p. 212] put it, we are describing a rhinoceros as a cross between a dragon and a unicorn.

The sum of the bond strengths is greater for an aromatic hydrocarbon than for any one of its resonance forms. The difference is called the resonance energy of the $\pi$-bond. The resonance energy is an increasing function of the number of resonance forms, which is just the number $m$ of perfect matchings of the associated graph. Calculating $m$ can be done efficiently if the graph has a Pfaffian orientation. (See Theorem 16 on page 35.)

The nature of $\pi$-bonds causes aromatic hydrocarbons to be physically planar, which results in the associated graphs being planar. Conveniently, all planar graphs have Pfaffian orientations, as shown by Kasteleyn $[\mathbf{1 5}, \mathbf{1 6}]$.

\section{Even Dicycles}

The study of even length dicycles in digraphs began with questions asked by Younger (communicated to Thomassen [2, p. 34] $)^{5}$, Levow [24], Seymour [40], and Lovász [26]. These questions concerned conditions on the connectivity, vertex degrees, and number of arcs which force a digraph to have an even dicycle. This research is surveyed in [30].

\footnotetext{
${ }^{5}$ Also communicated to the author at a later date.
} 
anthracene

84 kilocalories per mole<smiles>C1=CC2=CC=Cc3cccc2c3C=C1</smiles>

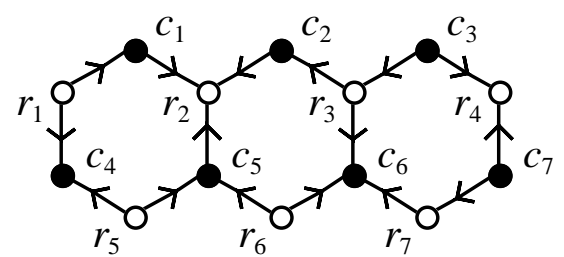$$
A=\left[\begin{array}{ccccccc}
1 & 0 & 0 & 1 & 0 & 0 & 0 \\
-1 & -1 & 0 & 0 & -1 & 0 & 0 \\
0 & 1 & -1 & 0 & 0 & 1 & 0 \\
0 & 0 & -1 & 0 & 0 & 0 & -1 \\
0 & 0 & 0 & 1 & 1 & 0 & 0 \\
0 & 0 & 0 & 0 & 1 & 1 & 0 \\
0 & 0 & 0 & 0 & 0 & 1 & -1
\end{array}\right]
$$$$
\operatorname{det}(A)=4
$$

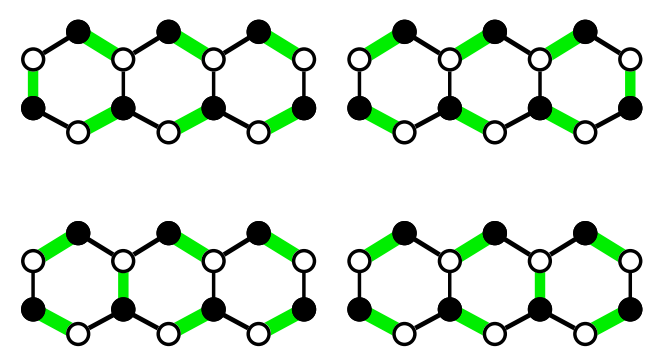

$\mathrm{H}$ hydrogen

C carbon $\pi$-bonds

Pfaffian orientations of the associated graphs<smiles>c1ccc2c(c1)ccc1ccccc12</smiles>

matrices of the oriented graphs

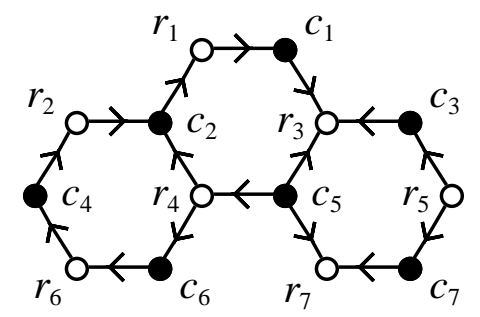
$\operatorname{det}(P)=5$
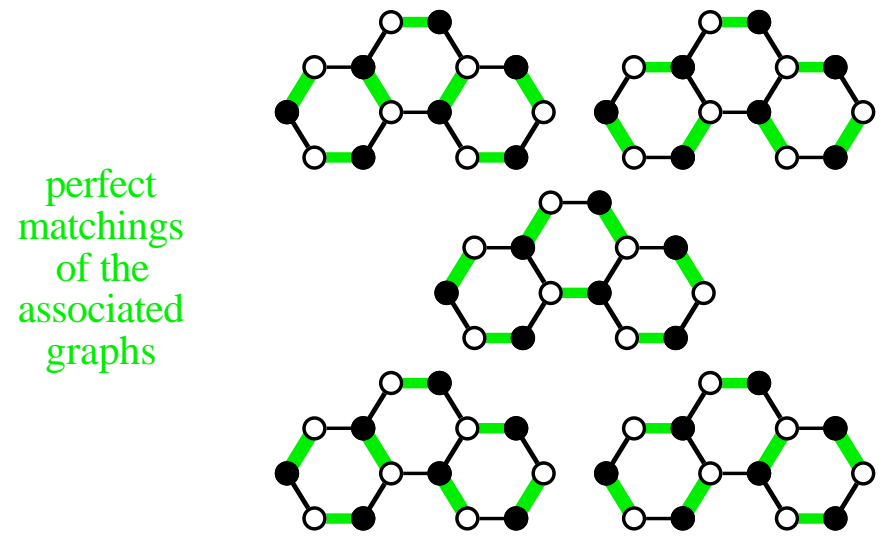

Figure 7: Organic Chemistry. 


\section{Versions}

In this section we discuss many versions of Pólya's permanent problem and show they are all algorithmically equivalent. All versions of Pólya's permanent problem will be given

as algorithmic questions. For each we are implicitly asking for a good characterization of some set $\mathcal{S}$ and a polynomial algorithm for determining membership in $\mathcal{S}$. Instances of one version can be naturally translated into instances of the other versions (F. 2, p. 6).

Let $A$ be a square matrix. The terms of $\operatorname{det}(A)$ are balanced if either all terms are zero, or there is a negative term and a positive term. The terms of $\operatorname{det}(A)$ are unbalanced if they are not balanced, that is, there is a nonzero term and all nonzero terms have the same sign.

A row of a matrix is balanced if either all its entries are zero, or it has a negative entry and a positive entry. The row is unbalanced if it is not balanced. A matrix is row-balanced if all its rows are balanced.

A square matrix $D=\left[d_{i j}\right]$ is diagonal if $d_{i j}=0$ for every $i$ and $j$ such that $i \neq j$. A matrix is nonzero if it has at least one nonzero entry.

Version 1. Given a square matrix $A$, are the terms of $\operatorname{det}(A)$ balanced?

Version 2. Is a given square matrix $A$ sign-nonsingular?

Version 3. Given a square matrix $A$, is there a nonzero diagonal $\{-1,0,1\}$-matrix $D$ such that $A D$ is row-balanced? (See equation $*$ on page 18.)

Version 4. Given a bipartite graph $G$ with a $\{-1,1\}$-edge weighting $w$ and a perfect matching $M$, are all $M$-alternating cycles $w$-unbalanced?

Version 5. Given a bipartite graph $G$ with a $\{-1,1\}$-edge weighting $w$, is $w$ unbalanced?

The next theorem shows versions 1 to 5 are equivalent. The equivalence between versions 1 and 2 is found in the work of Bassett, Maybee, and Quick [1], Samuelson [38], and Lancaster [21]. Klee, Ladner, and Manber [17] showed versions 2 and 3 are equivalent. Kasteleyn [16] showed versions 4 and 5 are equivalent.

Theorem 4 If $(G, w)$ is the weighted bipartite graph of a square real matrix $A=\left[a_{i j}\right]$, then the following statements are equivalent.

a) The terms of $\operatorname{det}(A)$ are balanced.

b) Let $\mathcal{E}$ be the set of all matrices with the same sign pattern as $A$. Either all matrices in $\mathcal{E}$ are singular, or $\mathcal{E}$ contains matrices with negative, positive, and zero determinant.

c) A is not sign-nonsingular.

d) There exists a nonzero diagonal $\{-1,0,1\}$-matrix $D$ such that $A D$ is row-balanced.

e) For every perfect matching $M$ of $G$, there exists a w-balanced $M$-alternating cycle.

f) Either $G$ has no perfect matching, or there exists a w-balanced $M$-alternating cycle for some perfect matching $M$ of $G$. 


$$
\begin{aligned}
& {\left[\begin{array}{lll}
a_{11} & a_{12} & 0
\end{array}\right] \quad \operatorname{det}(A)=a_{11} a_{22} a_{33}-a_{12} a_{21} a_{33}+a_{12} a_{23} a_{31}} \\
& p(x)=\operatorname{det}([1-x] B+x C)=896 x^{3}-1056 x^{2}+1454 x-575 \\
& B=\left[\begin{array}{ccc}
-1 & 9 & 0 \\
-1 & 1 & -7 \\
9 & 0 & -1
\end{array}\right] \\
& p(0)=\operatorname{det}(B)=-575 \\
& \frac{1}{2} B+\frac{1}{2} C=\left[\begin{array}{ccc}
-5 & 5 & 0 \\
-1 & 5 & -4 \\
5 & 0 & -5
\end{array}\right] \\
& C=\left[\begin{array}{ccc}
-9 & 1 & 0 \\
-1 & 9 & -1 \\
1 & 0 & -9
\end{array}\right] \\
& p\left(\frac{1}{2}\right)=\operatorname{det}\left(\frac{1}{2} B+\frac{1}{2} C\right)=0 \\
& p(1)=\operatorname{det}(C)=719
\end{aligned}
$$

Figure 8: An example for $(\mathrm{a} \Rightarrow \mathrm{b})$.

Proof. We prove $(\mathrm{a} \Rightarrow \mathrm{b} \Rightarrow \mathrm{c} \Rightarrow \mathrm{a}$ ) and $(\mathrm{c} \Rightarrow \mathrm{d} \Rightarrow \mathrm{e} \Rightarrow \mathrm{f} \Rightarrow \mathrm{c})$.

$(\mathbf{a} \Rightarrow \mathbf{b})$. Suppose the terms of $\operatorname{det}(A)$ are balanced. Then either all terms of $\operatorname{det}(A)$ are zero, or $\operatorname{det}(A)$ has a positive and a negative term. If all terms of $\operatorname{det}(A)$ are zero, then the same is true for all matrices in $\mathcal{E}$; and so all matrices in $\mathcal{E}$ are singular.

Suppose $\operatorname{det}(A)$ has a positive and a negative term (F. 8). Choose a negative term $\operatorname{sgn}(\sigma) \prod_{i=1}^{n} a_{i \sigma(i)}$. If $B=\left[b_{i j}\right]$ is in $\mathcal{E}$, then $\operatorname{sgn}(\sigma) \prod_{i=1}^{n} b_{i \sigma(i)}$ is also negative. If we choose $B$ so that $b_{1 \sigma(1)}, \ldots, b_{n \sigma(n)}$ have large absolute values relative to the other entries of $B$, then $\operatorname{sgn}(\sigma) \prod_{i=1}^{n} b_{i \sigma(i)}$ will dominate $\operatorname{det}(B)$; and so $\operatorname{det}(B)<0$. Similarly, we can use a positive term of $\operatorname{det}(A)$ to choose a matrix $C$ in $\mathcal{E}$ such that $\operatorname{det}(C)>0$.

Consider the continuous polynomial function

$$
p(x)=\operatorname{det}[(1-x) B+x C] .
$$

Since $p(0)=\operatorname{det}(B)<0$ and $p(1)=\operatorname{det}(C)>0$, the Intermediate Value Theorem implies $p(z)=0$ for some $z$ between 0 and 1 . Then $(1-z) B+z C$ is singular because $\operatorname{det}[(1-z) B+z C]=0$. Furthermore, $(1-z) B+z C$ has the same sign pattern as $B$ and $C$ because it is a convex combination of them; and so $(1-z) B+z C$ is in $\mathcal{E}$.

$(\mathbf{b} \Rightarrow \mathbf{c})$. This implication is trivial.

$(\mathbf{c} \Rightarrow \mathbf{a})$. We will prove $(\neg \mathrm{a} \Rightarrow \neg \mathrm{c})$. Suppose the terms of $\operatorname{det}(A)$ are unbalanced. Then $\operatorname{det}(A)$ has a nonzero term, and all nonzero terms are positive (respectively, negative). It follows that for every matrix $E$ in $\mathcal{E}$, $\operatorname{det}(E)$ has a nonzero term, and all nonzero terms are positive (respectively, negative). Therefore, all matrices in $\mathcal{E}$ have positive (respectively, negative) determinant. Thus, every matrix in $\mathcal{E}$ is nonsingular, that is, $A$ is sign-nonsingular. 
(c $\Rightarrow \mathbf{d})$. Suppose $A=\left[a_{i j}\right]$ is not sign-nonsingular. Then there exists a singular matrix $S=\left[s_{i j}\right]$ in $\mathcal{E}$. Choose a nonzero $n \times 1$ matrix $\bar{z}=\left[z_{i}\right]$ such that $S \bar{z}=\overline{0}$, and let $C=\left[c_{i j}\right]$ be the diagonal matrix such that $c_{i i}=z_{i}$ for $i=1, \ldots, n$. Here is an example.

$$
A=\left[\begin{array}{cccc}
1 & 1 & 0 & 1 \\
0 & 0 & -1 & 0 \\
-1 & 1 & 0 & 1 \\
1 & 1 & 0 & -1
\end{array}\right] \quad S=\left[\begin{array}{cccc}
2 & 4 & 0 & 2 \\
0 & 0 & -1 & 0 \\
-1 & 1 & 0 & 1 \\
5 & 1 & 0 & -1
\end{array}\right] \quad \bar{z}=\left[\begin{array}{c}
-1 \\
2 \\
0 \\
-3
\end{array}\right] \quad C=\left[\begin{array}{cccc}
-1 & 0 & 0 & 0 \\
0 & 2 & 0 & 0 \\
0 & 0 & 0 & 0 \\
0 & 0 & 0 & -3
\end{array}\right]
$$

Then $\overline{0}=S \bar{z}=S(C \overline{1})=(S C) \overline{1}$. Thus, the sum of the entries in any row of $S C$ is 0 . Hence, $S C$ is row-balanced.

$$
\overline{0}=S C \overline{1}=\left[\begin{array}{cccc}
2 & 4 & 0 & 2 \\
0 & 0 & -1 & 0 \\
-1 & 1 & 0 & 1 \\
5 & 1 & 0 & -1
\end{array}\right]\left[\begin{array}{cccc}
-1 & 0 & 0 & 0 \\
0 & 2 & 0 & 0 \\
0 & 0 & 0 & 0 \\
0 & 0 & 0 & -3
\end{array}\right]\left[\begin{array}{l}
1 \\
1 \\
1 \\
1
\end{array}\right]=\left[\begin{array}{cccc}
-2 & 8 & 0 & -6 \\
0 & 0 & 0 & 0 \\
1 & 2 & 0 & -3 \\
-5 & 2 & 0 & 3
\end{array}\right]\left[\begin{array}{l}
1 \\
1 \\
1 \\
1
\end{array}\right]
$$

Let $D=\left[d_{i j}\right]$ be the diagonal $\{-1,0,1\}$-matrix with the same sign pattern as $C$. Since $C$ is nonzero, so is $D$. Since $S$ and $A$ have the same sign pattern, and $C$ and $D$ have the same sign pattern, the $(i, j)$-entry $s_{i j} c_{j j}$ of $S C$ has the same sign as the $(i, j)$-entry $a_{i j} d_{j j}$ of $A D$ for $i=1, \ldots, n$ and $j=1, \ldots, n$. Hence, $S C$ and $A D$ have the same sign pattern. Therefore, $A D$ is also row-balanced.

$$
A D=\left[\begin{array}{cccc}
1 & 1 & 0 & 1 \\
0 & 0 & -1 & 0 \\
-1 & 1 & 0 & 1 \\
1 & 1 & 0 & -1
\end{array}\right]\left[\begin{array}{cccc}
-1 & 0 & 0 & 0 \\
0 & 1 & 0 & 0 \\
0 & 0 & 0 & 0 \\
0 & 0 & 0 & -1
\end{array}\right]=\left[\begin{array}{cccc}
-1 & 1 & 0 & -1 \\
0 & 0 & 0 & 0 \\
1 & 1 & 0 & -1 \\
-1 & 1 & 0 & 1
\end{array}\right]=B
$$

$(\mathbf{d} \Rightarrow \mathbf{e})$. Suppose there exists a nonzero diagonal $\{-1,0,1\}$-matrix $D=\left[d_{i j}\right]$ such that $A D=B$ is row-balanced. Suppose $M$ is a perfect matching of $G$ (F. 9). Let $(H, u)$ be the weighted bipartite graph of $B$. Let $r_{1}, \ldots, r_{n}$ be the row vertices and $c_{1}, \ldots, c_{n}$ be the column vertices of both $G$ and $H$. Then $H$ is obtained from $G$ by removing all edges incident with vertices $c_{j}$ such that $d_{j j}=0$. Furthermore, $u$ is obtained from the restriction $w_{r}$ of $w$ to $E(H)$ by switching at all the vertices $c_{j}$ such that $d_{j j}=-1$.
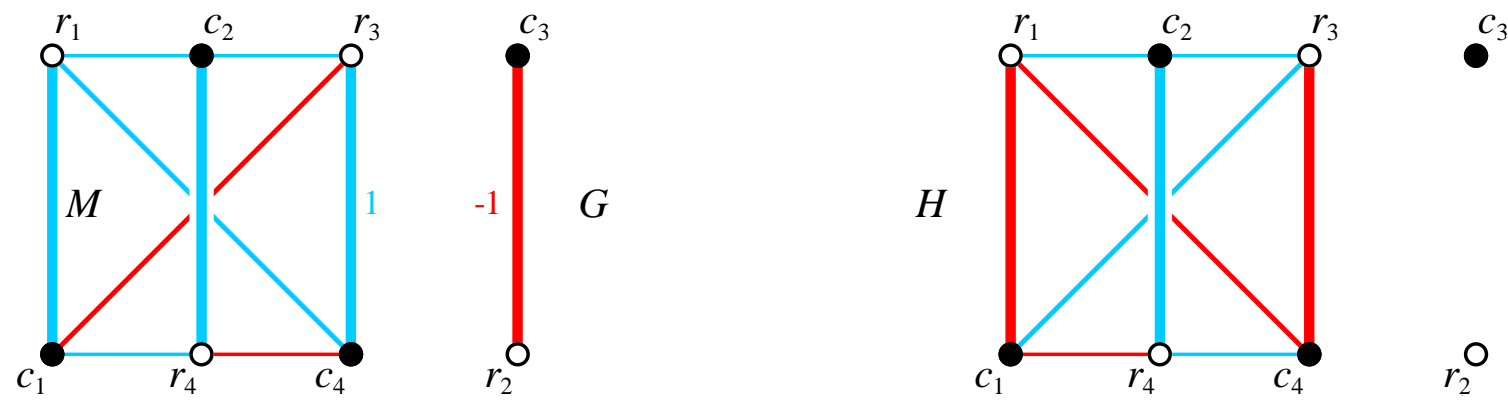

Figure 9: An example for $(\mathrm{d} \Rightarrow \mathrm{e})$. 

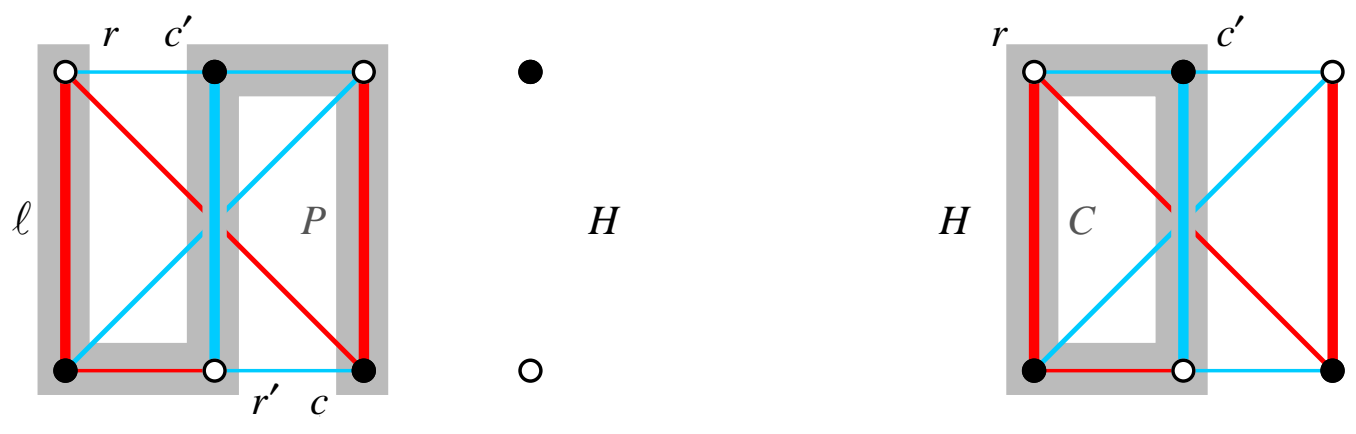

Figure 10: The example for $(\mathrm{d} \Rightarrow \mathrm{e})$ continued.

\section{Call a path $P$ of $H$ useful if}

- its origin is a column vertex,

- its terminus is a row vertex,

- it is well-fitted to $M$, and

- $u(e)=-u(f)$ whenever $e \in M$ and $e$ immediately precedes $f$ on $P$.

Since $D$ is nonzero, $d_{j j} \neq 0$ for some $j$ in $\{1, \ldots, n\}$. Then all the edges of $G$ incident with $c_{j}$ are also edges of $H$. In particular, $H$ includes the edge in $M$ incident with $c_{j}$. Hence, $H$ has a useful path of length 1 . Now choose a useful $(c, r)$-path $P$ of $H$ having maximum length (F. 10).

Since $B$ is row-balanced, either the row vertex $r$ of $H$ has degree 0 , or it is incident with both an edge of weight -1 and an edge of weight 1 . Since the last edge $\ell$ of $P$ is incident with $r$, we have the second possibility; and so we can choose an edge $r c^{\prime}$ of $H$ such that $u\left(r c^{\prime}\right)=-u(\ell)$. Since $r c^{\prime}$ is an edge of $H$, all edges of $G$ incident with the column vertex $c^{\prime}$ are edges of $H$. In particular, $H$ includes the edge $c^{\prime} r^{\prime}$ in $M$ incident with $c^{\prime}$. If $c^{\prime}$ is not on $P$, then $P+r c^{\prime} r^{\prime}$ is a useful path which is longer than $P$, and we have contradicted the maximality of $P$. Hence, $c^{\prime}$ is on $P$.

Now consider the $M$-alternating cycle $C=P\left[c^{\prime}, r\right]+r c^{\prime}$. For every edge $e$ in $E(C) \cap M$, if $f$ is the next edge on $C$, then $u(e)=-u(f)$. It follows that $u$ gives half the edges of $C$ weight 1 , and the other half weight -1 . This implies $C$ is $u$-balanced.

For an alternating cycle, the property of being balanced is not changed by switching; and so this property is invariant under equivalence. Since $C$ is $u$-balanced, and $u$ is equivalent to $w_{r}$, this invariance implies $C$ is also $w_{r}$-balanced. It then trivially follows that $C$ is $w$-balanced.

$(\mathbf{e} \Rightarrow \mathbf{f})$. This implication is trivial.

( $\mathbf{f} \Rightarrow \mathbf{c})$. Suppose $G$ has no perfect matching. For every permutation $\sigma$ in $S_{n}$, the term $\operatorname{sgn}(\sigma) \prod_{i=1}^{n} a_{i, \sigma(i)}$ of $\operatorname{det}(A)$ is nonzero if and only if $\left\{r_{1} c_{\sigma(1)}, \ldots, r_{n} c_{\sigma(n)}\right\}$ is a perfect matching of $G$. Hence, all terms of $\operatorname{det}(A)$ are zero. Since $A$ itself is singular, $A$ is not sign-nonsingular. 
Suppose $G$ has a perfect matching $M$ and a $w$-balanced $M$-alternating cycle $C$. Let $A^{\prime}$ be the $\{-1,0,1\}$-matrix with the same sign pattern as $A$. Relabel the row and column vertices of $G$ with $x_{1}, \ldots, x_{n}$ and $y_{1}, \ldots, y_{n}$, respectively, so that $M=\left\{x_{1} y_{1}, \ldots, x_{n} y_{n}\right\}$ and $C=y_{1} x_{1} y_{2} x_{2} \cdots y_{k} x_{k} y_{1}$. With the new vertex names, $(G, w)$ becomes the weighted bipartite graph of a matrix $B=\left[b_{i j}\right]$ which is obtained from $A^{\prime}$ by permuting rows and columns. Let $P$ and $Q$ be the permutation matrices such that $P B Q=A^{\prime}$.

Next we consider the following terms of $\operatorname{det}(B)$ :

$$
\begin{aligned}
s & =\operatorname{sgn}[i] \cdot b_{11} b_{22} \cdots b_{k k} \cdot b_{k+1, k+1} \cdots b_{n n} \text { and } \\
t & =\operatorname{sgn}[(1,2, \ldots, k)] \cdot b_{12} b_{23} \cdots b_{k-1, k} b_{k 1} \cdot b_{k+1, k+1} \cdots b_{n n} .
\end{aligned}
$$

Since $B$ is a $\{-1,0,1\}$-matrix, and $C$ is $w$-balanced, we have

$$
\begin{aligned}
s t & =\operatorname{sgn}[(1,2, \ldots, k)] \cdot b_{11} b_{22} \cdots b_{k k} \cdot b_{12} b_{23} \cdots b_{k-1, k} b_{k 1} \\
& =\operatorname{sgn}[(1,2)(1,3) \cdots(1, k)] b_{11} b_{12} b_{22} b_{23} \cdots b_{k-1, k} b_{k k} b_{k 1} \\
& =(-1)^{k-1} w\left(y_{1} x_{1}\right) w\left(x_{1} y_{2}\right) w\left(y_{2} x_{2}\right) w\left(x_{2} y_{3}\right) \cdots w\left(x_{k-1} y_{k}\right) w\left(y_{k} x_{k}\right) w\left(x_{k} y_{1}\right) \\
& =(-1)^{k-1} w(C)=(-1)^{k-1}(-1)^{k}=-1 .
\end{aligned}
$$

Therefore, $s$ and $t$ have opposite signs, and so $B$ satisfies statement (a). We have already proven (a) implies (c); and so $B$ satisfies (c). Hence, there exists a singular matrix $S$ with the same sign pattern as $B$. Then $P S Q$ is a singular matrix with the same sign pattern as $P B Q=A^{\prime}$. Since $A^{\prime}$ and $A$ have the same sign pattern, $A$ is not sign-nonsingular.

Let $G$ be a bipartite graph with a $\{-1,1\}$-edge weighting $w$. If we wish to show $w$ is unbalanced, then Theorem 4 implies that we only need to verify all $M$-alternating cycles are $w$-unbalanced for some fixed perfect matching $M$. The theorem also implies that if $M$ and $M^{\prime}$ are perfect matchings, then there is a $w$-balanced $M$-alternating cycle if and only if there is a $w$-balanced $M^{\prime}$-alternating cycle.

Lemma 5 Suppose $D$ is a digraph with a $\{-1,1\}$-arc weighting $\vec{w},(G, w)$ is the weighted bipartite graph of $(D, \vec{w})$, and $M$ is the perfect matching of $G$ corresponding to $V(D)$. Then $D$ has a $\vec{w}$-positive dicycle if and only if $G$ has a $w$-balanced $M$-alternating cycle.

Proof. (F. 2, p. 6, D and $G$ ). Suppose $\vec{C}=v_{1} v_{2} \cdots v_{k} v_{1}$ is a dicycle of $D . \vec{C}$ corresponds to an $M$-alternating cycle $C=c_{1} r_{1} c_{2} r_{2} c_{3} \cdots c_{k} r_{k} c_{1}$ of $G$, where $v_{i}$ corresponds to $c_{i} r_{i}$ in $M$ for $i=1,2, \ldots, k$. By definition, $w(e)=-1$ for every edge $e$ in $M$. Then

$$
\begin{aligned}
w(C) & =\left[w\left(c_{1} r_{1}\right) w\left(c_{2} r_{2}\right) \cdots w\left(c_{k} r_{k}\right)\right] \cdot\left[w\left(r_{1} c_{2}\right) w\left(r_{2} c_{3}\right) \cdots w\left(r_{k} c_{1}\right)\right] \\
& =(-1)^{k} \cdot\left[\vec{w}\left(v_{1} v_{2}\right) \vec{w}\left(v_{2} v_{3}\right) \cdots \vec{w}\left(v_{k} v_{1}\right)\right]=(-1)^{k} \cdot \vec{w}(\vec{C}) .
\end{aligned}
$$

Hence, $\vec{w}(\vec{C})=1$ if and only if $w(C)=(-1)^{k}$, that is, $\vec{C}$ is $\vec{w}$-positive if and only if $C$ is $w$-balanced. 
Version 6. Is a $\{-1,1\}$-arc weighting of a digraph negative?

Next we show versions 4 and 6 are equivalent. Suppose $G$ is a bipartite graph with a $\{-1,1\}$-edge weighting $u$ and a perfect matching $M$. Let $w$ be a weighting equivalent to $u$ which assigns -1 to all edges in $M$. Then $(G, w)$ corresponds to a weighted digraph $(D, \vec{w})$. For an $M$-alternating cycle, the property of being unbalanced is invariant under equivalence; and so all $M$-alternating cycles of $G$ are $u$-unbalanced if and only if they are all $w$-unbalanced. Furthermore, Lemma 5 implies that all $M$-alternating cycles of $G$ are $w$-unbalanced if and only if all dicycles of $D$ are $\vec{w}$-negative.

Bassett, Maybee, and Quick [1] proved the equivalence between the relatively distant versions 2 and 6. Thomassen [46] gave a polynomial algorithm for version 6 when it is restricted to planar digraphs. In the same paper he observed that, other than $D_{7}$ (F. 4), the only strongly 2 -connected digraphs with negative $\{-1,1\}$-arc weightings "that I know of are obtained from planar digraphs in a simple way. This suggests that perhaps the even dicycle problem can be reduced to the planar case." When the Main Theorem is translated into digraphs (not easy), we see his suggestion is true.

Version 7. Does a given digraph have a negative $\{-1,1\}$-arc weighting?

A dipath $P$ is an ear dipath of a digraph $H$ if the ends of $P$ are on $H$, but no arc or intermediate vertex of $P$ is on $H$. We also call a dicycle an ear dipath of $H$ if it has a unique vertex on $H$. An ear decomposition of a digraph $D$ is a sequence $D_{1}, D_{2}, \ldots, D_{n}$ of subdigraphs of $D$ satisfying the following conditions (F. 11).

- $D_{1}$ is a $K_{1}$.

- there exists an ear dipath $P_{i}$ of $D_{i-1}$ such that $D_{i-1}+P_{i}=D_{i}$ for $i=2, \ldots, n$.

- $D_{n}=D$.

Next we follow the work of Little [25] and Seymour and Thomassen [42] to show that the slightly different versions 6 and 7 are equivalent. The next result is implicit in Hetyei [13] and explicit in Lovász and Plummer [27]. Hartfiel [12] proved a matrix version.

Lemma 6 A digraph has an ear decomposition if and only if it is strongly connected. There is a polynomial algorithm for constructing an ear decomposition when one exists.

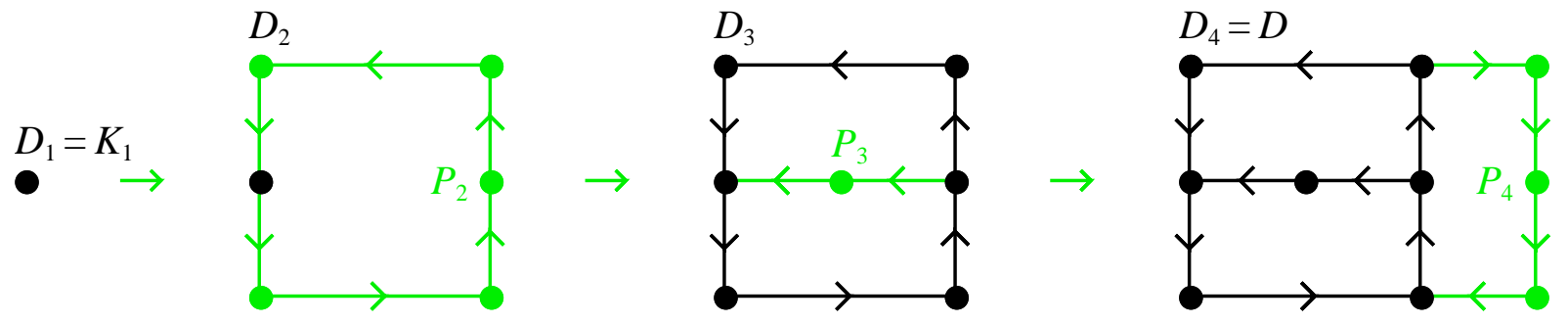

Figure 11: An ear decomposition of a strongly connected digraph $D$. 
Theorem 7 Suppose we know a strongly connected digraph $D$ has a negative $\{-1,1\}$-arc weighting $\vec{u}$, but we do not know the weights assigned to the arcs by $\vec{u}$. Then the following statements hold.

a) There is a polynomial algorithm for constructing an explicit negative $\{-1,1\}$-arc weighting of $D$.

b) Given an arbitrary $\{-1,1\}$-arc weighting of $D$, there is a polynomial algorithm for determining if it is negative.

c) All negative $\{-1,1\}$-arc weightings of $D$ are equivalent.

Proof. $D$ has an ear decomposition $D_{1}, \ldots, D_{n}$ by Lemma 6 . Suppose $2 \leq i \leq n$. Let $P_{i}$ be the dipath such that $D_{i-1}+P_{i}=D_{i} . D_{1}, \ldots, D_{i}$ is an ear decomposition of $D_{i}$; and so $D_{i}$ is strongly connected by Lemma 6 . Hence, we can choose a dicycle $C_{i}$ of $D_{i}$ which uses $P_{i}$. In order to prove the theorem, we first need to prove the following statements are equivalent for a $\{-1,1\}$-arc weighting $\vec{w}$ of $D$.

1) $\vec{w}$ is equivalent to $\vec{u}$.

2) $\vec{w}$ is negative.

3) $\vec{w}\left(C_{i}\right)=-1$, for $i=2, \ldots n$.

Since $u$ is negative, (1) implies (2). Trivially, (2) implies (3).

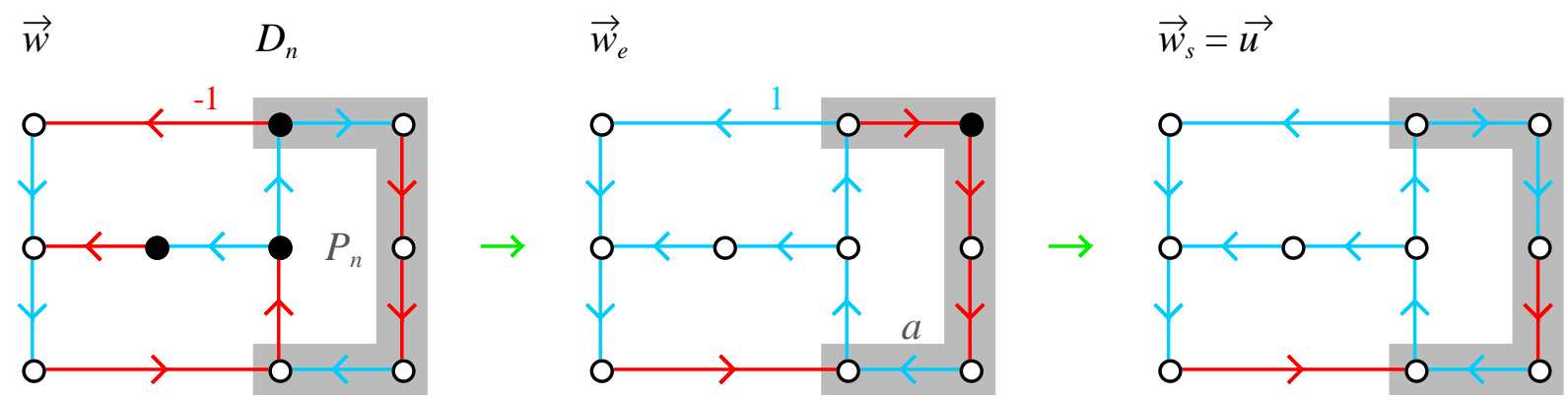

Figure 12: An example for the proof.

Suppose (3) holds. We will proof (1) holds by induction on $n$. If $n=1$, then $D \cong K_{1}$ and (1) is trivially true. Suppose $n \geq 2$. The restrictions of $\vec{w}$ and $\vec{u}$ to $A\left(D_{n-1}\right)$ are equivalent by the induction hypothesis. It follows that $\vec{w}$ is equivalent to a weighting $\vec{w}_{e}$ such that the restrictions of $\vec{w}_{e}$ and $\vec{u}$ to $A\left(D_{n-1}\right)$ are equal (F. 12). Let $a$ be the last arc of $P_{n}$. By switching on some of the intermediate vertices of $P_{n}$, we can get a weighting $\vec{w}_{s}$ from $\vec{w}_{e}$ such that the restrictions of $\vec{w}_{s}$ and $\vec{u}$ to $A(D-a)$ are equal. Hence,

$$
\vec{w}_{s}\left(C_{n}-a\right)=\vec{u}\left(C_{n}-a\right) \text { and } \vec{w}_{s}\left(C_{n}\right)=\vec{w}\left(C_{n}\right)=-1=\vec{u}\left(C_{n}\right) .
$$

Then $\vec{w}_{s}(a)=\vec{u}(a)$. It follows that $\vec{w}_{s}$ and $\vec{u}$ are equal; and so (1) holds. 
The results of the lemma are now easy. Construct a $\{-1,1\}$-arc weighting $\vec{u}_{i}$ of $D_{i}$ for $i=1, \ldots, n$ as follows. Let $\vec{u}_{1}$ be the empty function. For $i=2, \ldots, n$, construct $\vec{u}_{i}$ by extending $\vec{u}_{i-1}$ to $D_{i}$ so that $\vec{u}_{i}\left(C_{i}\right)=-1$. Then $\vec{u}_{n}\left(C_{i}\right)=-1$ for $i=2, \ldots, n$. It follows that $\vec{u}_{n}$ is negative; and so statement (a) holds. As for (b), in order to determine if a given weighting is negative, we just need to determine if $C_{2}, \ldots, C_{n}$ are negative. Finally, (c) holds because we have shown that all negative weightings are equivalent to $\vec{u}$.

Now we can show the equivalence of versions 6 and 7 . Suppose we have a polynomial algorithm $\mathcal{A}_{6}$ for answering version 6 , and suppose we have an instance of version 7 , that is, we want to determine if a given digraph $D$ has a negative $\{-1,1\}$-arc weighting. A digraph has such a weighting if and only if all its strong components have such a weighting; and so we may assume $D$ is strongly connected. Construct a $\{-1,1\}$-arc weighting $\vec{u}_{n}$ using the polynomial algorithm of Theorem 7a. Apply $\mathcal{A}_{6}$ to determine if $\vec{u}_{n}$ is negative. Since $\vec{u}_{n}$ is negative if and only if $D$ has a negative weighting by Theorem 7 a, we determine if $D$ has a negative weighing.

Suppose we have a polynomial algorithm $\mathcal{A}_{7}$ for answering version 7 , and suppose we have an instance of version 6 , that is, we want to determine if a given $\{-1,1\}$-arc weighting $\vec{w}$ of a digraph $D$ is negative. As in the previous paragraph, we may assume $D$ is strongly connected. Apply $\mathcal{A}_{7}$ to determine if $D$ has a negative weighting. If a negative weighting exists, then we use Theorem $7 \mathrm{~b}$ to determine if $\vec{w}$ is negative. If there is no negative weighting, then obviously $\vec{w}$ is not negative.

Version 8. Does a given bipartite graph have an unbalanced $\{-1,1\}$-edge weighting?

Version 8 can be translated into version 7 . Let $G$ be a bipartite graph. There is a polynomial algorithm to determine if $G$ has a perfect matching $M$ (See Lovász and Plummer [28].) If $M$ does not exist, then by definition $G$ has no unbalanced weighting. If $M$ exists, then $G$ corresponds to a digraph $D$, where $M$ corresponds to $V(D)$. By Lemma 5 we know that $D$ has a negative $\{-1,1\}$-arc weighting if and only if $G$ has a $\{-1,1\}$-edge weighting such that all $M$-alternating cycles are unbalanced, which in turn is equivalent to $G$ having an unbalanced weighting by Theorem 4. Similarly, version 7 can be translated into version 8 .

Next we follow the work of Thomassen [45] to show versions 7 and 8 can be restricted to looking at only strongly 2-connected digraphs and braces, respectively.

Suppose digraph $D$ is strongly connected but not strongly 2-connected, and $\nu(D) \geq 3$. Then $D$ has a vertex $v$ such that $D-v$ is not strongly connected (F. 13, p. 24). It is easy to show $V(D-v)$ has a partition $\left\{V_{1}, V_{2}\right\}$ such that all arcs between $V_{1}$ and $V_{2}$ are outgoing arcs of $V_{1}$. For $i=1,2$, let $N_{i}$ be the set of vertices in $V_{i}$ which are incident with arcs from $V_{1}$ to $V_{2}$. Let

$$
D_{1}=D\left[V_{1} \cup\{v\}\right]+\left\{x v \mid x \in N_{1}\right\} \quad \text { and } \quad D_{2}=D\left[\{v\} \cup V_{2}\right]+\left\{v y \mid y \in N_{2}\right\} .
$$

We say $D_{1}$ and $D_{2}$ are reductions of $D($ at $v)$.

Lemma 8 (Thomassen [45]) If $D_{1}$ and $D_{2}$ are reductions of a digraph $D$, then $D$ has a negative $\{-1,1\}$-arc weighting if and only if $D_{1}$ and $D_{2}$ have such weightings. 

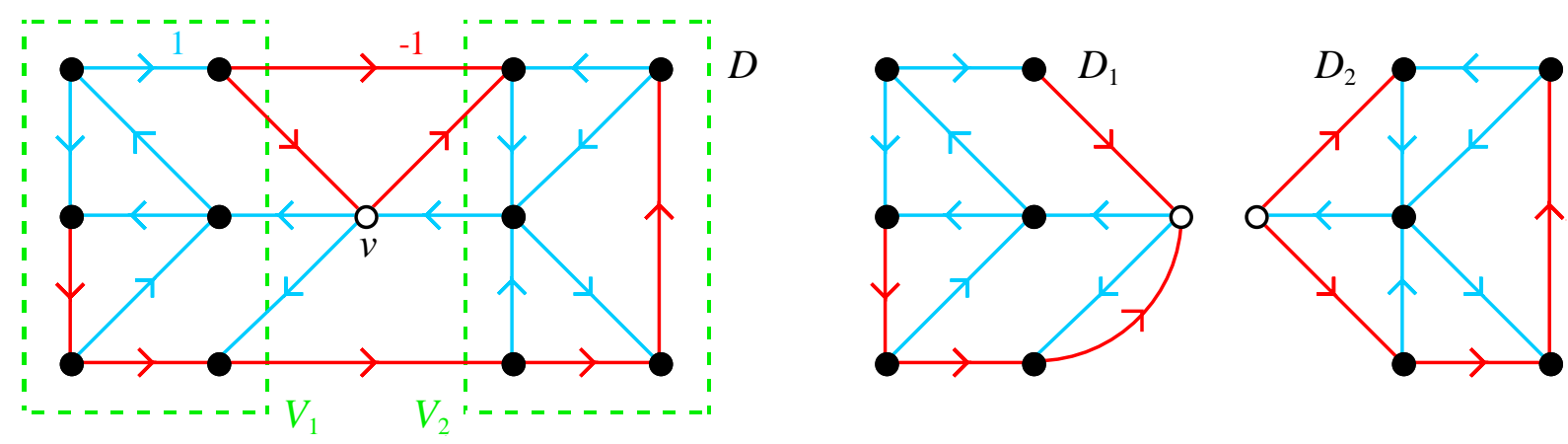

Figure 13: Reductions $D_{1}$ and $D_{2}$ of a digraph $D$ at a vertex $v$, and negative weightings of the three digraphs.

Lemma 8 has an equivalent version involving bipartite graphs [31, Lemma 26]. Version 7 can now be reduced to the following version using Lemma 8.

Version 7a. Does a given strongly 2-connected digraph have a negative $\{-1,1\}$-arc weighting?

Version 8a. Does a given brace have an unbalanced $\{-1,1\}$-edge weighting?

Suppose digraph $D$ corresponds to bipartite graph $G$. $G$ is a brace if and only if $D$ is strongly 2 -connected by Theorem 3. Furthermore, $G$ has an unbalanced $\{-1,1\}$-edge weighting if and only if $D$ has a negative $\{-1,1\}$-arc weighting by Lemma 5 . Hence, version $7 \mathrm{a}$ and $8 \mathrm{a}$ are equivalent.

Note that version $8 \mathrm{a}$ is the one solved by the Main Theorem.

Version 9. Given a square matrix $A$, does there exist a sign-nonsingular matrix $L$ with the same dimensions and support as $A$ ?

Let $A$ be a square matrix, and suppose $L$ is a matrix with the same dimensions and support as $A$. Let $(G, w)$ be the weighted bipartite graph of $L$. Then $w$ is an unbalanced $\{-1,1\}$-edge weighting of $G$ if and only if $L$ is sign-nonsingular by Theorem 4 . Therefore, versions 8 and 9 are equivalent.

Version 10. Can Pólya's method (p. 11) be used to calculate the permanent of a given square matrix?

Given $m \times n$ matrices $A=\left[a_{i j}\right]$ and $B=\left[b_{i j}\right]$, we define their Hadamard product $A * B$ to be the $m \times n$ matrix $\left[a_{i j} b_{i j}\right]$. Changing the signs of the entries of a matrix $A$ is equivalent to taking the Hadamard product $A * L$ of $A$ and some $\{-1,0,1\}$-matrix $L$ with the same dimensions and support as $A$. We now reformulate version 10 . Given an $n \times n$ matrix $A$, does there exist an $n \times n\{-1,0,1\}$-matrix $L$ with the same support as $A$ such that corresponding terms of perm $(A)$ and $\operatorname{det}(A * L)$ are equal. The next theorem gives us a condition for when such an $L$ exists. 
Lemma 9 Let $A=\left[a_{i j}\right]$ be an $n \times n$ matrix and let $L=\left[\ell_{i j}\right]$ be an $n \times n\{-1,0,1\}$-matrix with the same support as $A$. Then corresponding terms of perm $(A)$ and $\operatorname{det}(A * L)$ are equal if and only if all nonzero terms of $\operatorname{det}(L)$ are equal to 1.

Proof. Suppose $\sigma$ is in $S_{n}$. Since $A$ and $L$ have the same support, either the three terms $\prod_{i=1}^{n} a_{i, \sigma(i)}, \operatorname{sgn}(\sigma) \prod_{i=1}^{n} a_{i, \sigma(i)} \ell_{i, \sigma(i)}, \quad$ and $\operatorname{sgn}(\sigma) \prod_{i=1}^{n} \ell_{i, \sigma(i)}$ are all equal to zero, or they are all nonzero. If they are all nonzero, then

$$
\prod_{i=1}^{n} a_{i, \sigma(i)}=\operatorname{sgn}(\sigma) \prod_{i=1}^{n} a_{i, \sigma(i)} \ell_{i, \sigma(i)} \Leftrightarrow \operatorname{sgn}(\sigma) \prod_{i=1}^{n} \ell_{i, \sigma(i)}=1 .
$$

The result now follows.

We will now show versions 9 and 10 are equivalent. Let $A$ be an $n \times n$ matrix. The polynomial algorithm for determining if a bipartite graph has a perfect matching can be used to determine if $\operatorname{det}(A)$ has a nonzero term. If all terms of $\operatorname{det}(A)$ are zero, then it is trivial to show that Pólya's method can be applied to $A$, and that there is no signnonsingular $n \times n$ matrix $L$ with the same support as $A$. Therefore, we may assume $\operatorname{det}(A)$ has a nonzero term.

Suppose Pólya's method can be applied to $A$. By Lemma 9 there exists an $n \times n$ $\{-1,0,1\}$-matrix $L$ with the same support as $A$ such that all nonzero terms of $\operatorname{det}(L)$ are equal to 1 . Since $\operatorname{det}(A)$ has a nonzero term, so does $\operatorname{det}(L)$. Then $L$ is sign-nonsingular by Theorem 4 . Therefore, there is a sign-nonsingular $n \times n$ matrix with the same support as $A$.

Conversely, suppose there exists a sign-nonsingular $n \times n$ matrix $L^{\prime}$ with the same support as $A$. By Theorem 4 all nonzero terms of $\operatorname{det}\left(L^{\prime}\right)$ have the same sign. If all the entries of some row of $L^{\prime}$ are multiplied by -1 to give a matrix $L^{\prime \prime}$, then the nonzero terms of $\operatorname{det}\left(L^{\prime \prime}\right)$ have the opposite sign as the nonzero terms of $\operatorname{det}\left(L^{\prime}\right)$. Hence, we may assume all nonzero terms of $\operatorname{det}\left(L^{\prime}\right)$ are positive. If $L$ is the $\{-1,0,1\}$-matrix with the same sign pattern as $L$, then all nonzero terms of $\operatorname{det}(L)$ will equal 1 . Therefore, Pólya's method can be applied to $A$ by Lemma 9 .

Next we discuss "unweighted" versions of Pólya's permanent problem. Suppose we have an instance of version 6 , that is, we want to determine if a given $\{-1,1\}$-arc weighting $\vec{w}$ of a digraph $D$ is negative. Equivalently, does $D$ have a $\vec{w}$-positive dicycle? Let $D^{\prime}$ be the digraph obtained from $D$ by replacing every arc $x y$ such that $\vec{w}(x y)=1$ by a dipath $x z y$ (F. 2, p. 6). There is a natural correspondence between the dicycles of $D$ and $D^{\prime}$. Let $C$ and $C^{\prime}$ be corresponding dicycles of $D$ and $D^{\prime}$, respectively. Then $C$ is $\vec{w}$-positive if and only if $C^{\prime}$ has even length. Hence, the instance of version 6 can be answered using a polynomial algorithm for the following version.

Version 11. Does a given digraph have a dicycle of even length?

Going in the other direction is easier. An instance of version 11 can be translated into an instance of version 6 by weighting every arc with -1 . Therefore, versions 6 and 11 are equivalent. There are six more "unweighted" versions. We denote the set of nonnegative real numbers by $\Re_{0}^{+}$. 
Version 12. Given a square $\left(\Re_{0}^{+}\right)$-matrix $A$, does $\operatorname{det}(A)=\operatorname{perm}(A)$ ?

Version 13. Given a square $\left(\Re_{0}^{+}\right)$-matrix $A$, are the terms of $\operatorname{det}(A)$ balanced?

Version 14. Is a given square $\left(\Re_{0}^{+}\right)$-matrix $A$ sign-nonsingular?

Version 15. Given a square $\left(\Re_{0}^{+}\right)$-matrix $A$, does there exist a nonzero diagonal $\{-1,0,1\}$-matrix $D$ such that $A D$ is row-balanced?

Version 16. Given a bipartite graph $G$ with a perfect matching $M$, does $G$ have an $M$-alternating cycle whose length is divisible by 4 ?

Version 17. Does a given bipartite graph $G$ have an alternating cycle whose length is divisible by 4 ?

The next theorem shows the "unweighted" versions are equivalent. It is proven using earlier results, or by modifying their proofs.

Theorem 10 (F. 4) Let $A$ be a square $\left(\Re_{0}^{+}\right)$-matrix such that $\operatorname{det}(A)$ has a positive term. Then the bipartite graph $G$ of $A$ has a perfect matching. Let $D$ be a digraph corresponding to $G$. The following statements are equivalent.

a) All dicycles of $D$ have odd length.

b) $\operatorname{Det}(A)=\operatorname{perm}(A)$.

c) All nonzero terms of $\operatorname{det}(A)$ are positive.

d) A is sign-nonsingular.

e) For every nonzero diagonal $\{-1,0,1\}$-matrix $D$, some row of $A D$ is unbalanced.

f) There exists a perfect matching $M$ of $G$ such that all $M$-alternating cycles have length congruent to 2 modulo 4.

g) All alternating cycles of $G$ have length congruent to 2 modulo 4.

Next we look at obstructions. In the context of version 7 , an obstruction is a minimal digraph which does not have a negative $\{-1,1\}$-arc weighting. The significance of this definition is that a digraph has a negative $\{-1,1\}$-arc weighting if and only if it does not have a subdigraph which is an obstruction. As in other graph problems involving obstructions, the goal is to characterize the set of obstructions. We first need some definitions.

Let $v$ be a vertex of a digraph $D(\mathrm{~F} .14 \mathrm{a}, \mathrm{p} .27)$. Let $D^{\prime}$ be the digraph obtain from $D-v$ by adding new vertices $v^{-}$and $v^{+}$, and then adding the arcs in

$$
\left\{z v^{-} \mid z v \in A(D)\right\} \cup\left\{v^{-} v^{+}\right\} \cup\left\{v^{+} z \mid v z \in A(D)\right\} .
$$

We say $D^{\prime}$ is obtained from $D$ by vertex splitting. If $D^{\prime \prime}$ is obtained from $D$ via a sequence of vertex splittings, then $D^{\prime \prime}$ is called a weak $D$. ( $D$ itself is also a weak $D$.) 
(a)

$D$
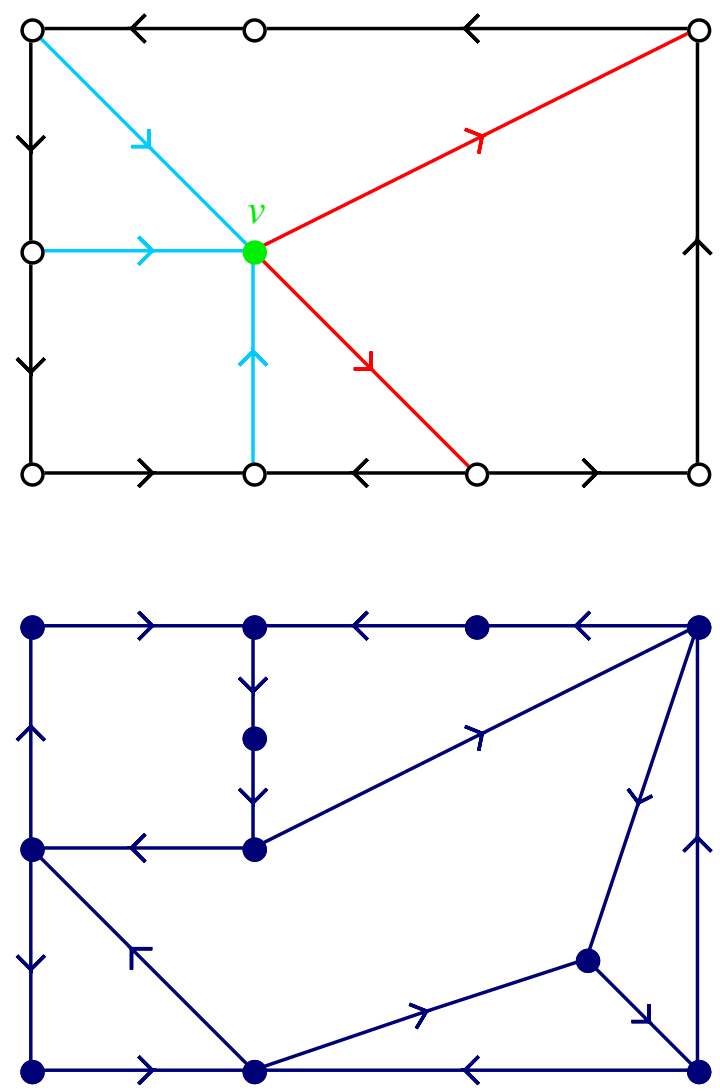

Weak $\overleftrightarrow{C}_{5}$
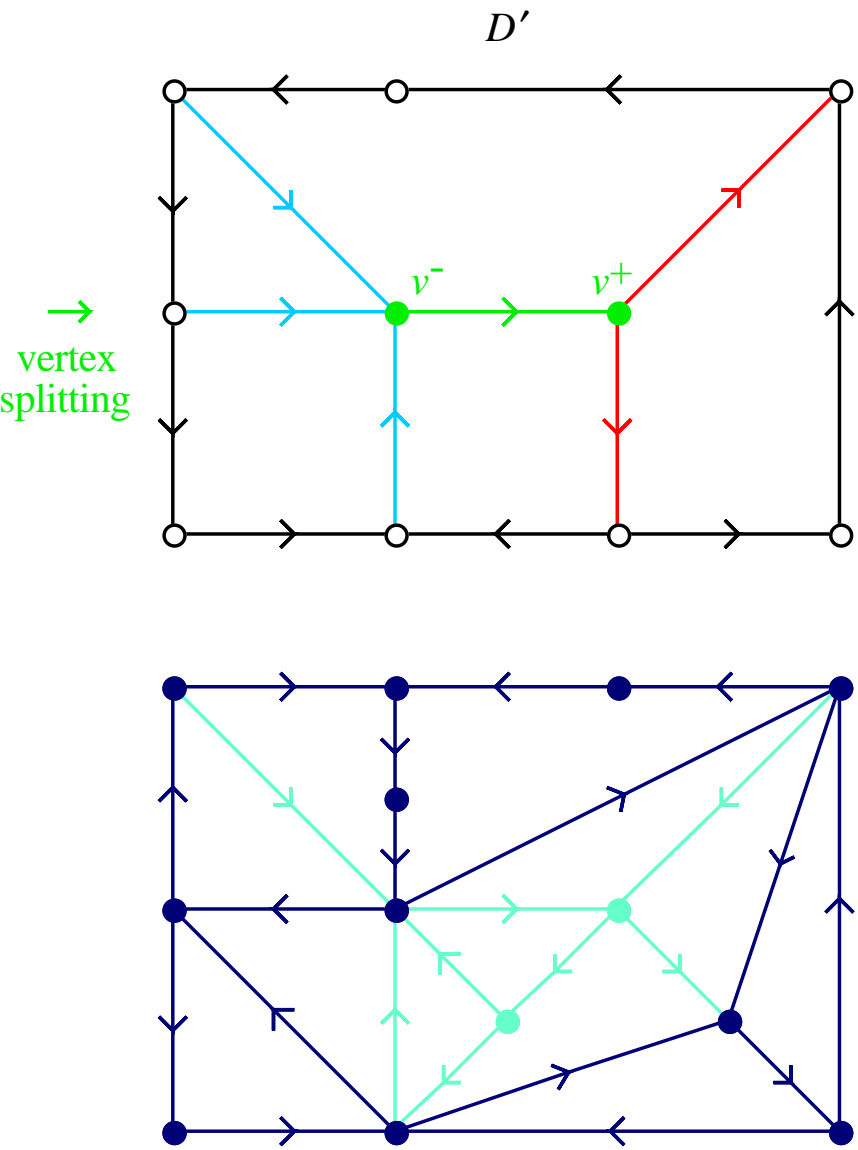

A digraph with a weak $\overleftrightarrow{C_{5}}$

(b)

Figure 14: Examples for vertex splitting and Theorem 11.

For $n \geq 3$, the double dicycle $\overleftrightarrow{C}_{n}$ is the symmetric digraph obtained from the $n$-cycle by replacing every edge $x y$ by the $\operatorname{arcs} x y$ and $y x$. If $n$ is odd, we say $\overleftrightarrow{C}_{n}$ is odd. The next result characterizes the obstructions to a digraph having a negative $\{-1,1\}$-arc weighting. We will prove it in Section 10 using the Main Theorem.

Theorem 11 (Seymour and Thomassen [42]) (F. 14b) A digraph has a weak odd $\overleftrightarrow{C}_{n}$ if and only if it does not have a negative $\{-1,1\}$-arc weighting.

Given Theorem 11, version 7 is equivalent to the following version.

Version 18. Does a given digraph have a weak odd $\overleftrightarrow{C}_{n}$ ? 
In the context of version 8, an obstruction is a bipartite graph $H$ with a perfect matching such that $H$ does not have an unbalanced $\{-1,1\}$-edge weighting, but every well-fitted proper subgraph does have an unbalanced $\{-1,1\}$-edge weighting.

Characterizing the set of obstructions for version 8 is done by the following theorem. For now we only prove it in the "easy" direction. This part of the theorem will be used later in proving the Main Theorem. In Section 10 we will then use the Main Theorem to prove Theorem 12 in the "difficult" direction.

Theorem 12 (Little [25]) If $G$ is a bipartite graph with a perfect matching, then $G$ has a well-fitted $K_{3,3}$ bisubdivision (F. 1, p. 3) if and only if $G$ does not have an unbalanced $\{-1,1\}$-edge weighting.

Proof $(\Rightarrow)$. We first show $K_{3,3}$ does not have an unbalanced $\{-1,1\}$-edge weighting. Suppose $w$ is an arbitrary $\{-1,1\}$-edge weighting of $K_{3,3}$. For every edge of $K_{3,3}$, there is a unique 4-cycle which is disjoint from the edge. Thus, $K_{3,3}$ has nine 4 -cycles $C_{1}, \ldots, C_{9}$. Each edge of $K_{3,3}$ is on four of these cycles. Hence,

$$
\prod_{i=1}^{9} w\left(C_{i}\right)=\prod_{e \in E\left(K_{3,3}\right)}[w(e)]^{4}=1 .
$$

Since 9 is odd, $w\left(C_{i}\right)=1$ for some $i$ in $\{1, \ldots, 9\}$. Then $C_{i}$ is a balanced alternating cycle of $K_{3,3}$. Therefore, $w$ is not unbalanced.

Next we use induction on the number of edges to show $K_{3,3}$ bisubdivisions do not have unbalanced $\{-1,1\}$-edge weightings. The induction basis is the previous paragraph. Suppose $K$ and $K^{\prime}$ are $K_{3,3}$ bisubdivisions such that $K^{\prime}$ is obtained from $K$ by replacing an edge $x y$ of $K$ by the path $x v u y$, that is, by subdividing $x y$ twice. Suppose $w^{\prime}$ is an arbitrary $\{-1,1\}$-edge weighting of $K^{\prime}$. Let $w$ be the $\{-1,1\}$-edge weighting of $K$ such that $w(x y)=-w^{\prime}(x v) \cdot w^{\prime}(v u) \cdot w^{\prime}(u y)$ and $w(e)=w^{\prime}(e)$ for every $e$ in $E(K)-\{x y\}$. By the induction hypothesis, $w$ is not unbalanced. Then either $K$ does not have a perfect matching, or $K$ has a perfect matching $M$ and $w$-balanced $M$-alternating cycle $C$. Since all $K_{3,3}$ bisubdivisions have perfect matchings, $C$ exists. Then $M$ and $C$ naturally correspond to a perfect matching $M^{\prime}$ and a $w^{\prime}$-balanced $M^{\prime}$-alternating cycle of $K^{\prime}$. Therefore, $w^{\prime}$ is not unbalanced.

Finally, suppose $G$ has a well-fitted $K_{3,3}$ bisubdivision $K$. Suppose $w$ is an arbitrary $\{-1,1\}$-edge weighting of $G$, and let $w_{r}$ be the restriction of $w$ to $E(K)$. Since $K$ has no unbalanced $\{-1,1\}$-edge weighting, $w_{r}$ is not unbalanced. Then either $K$ does not have a perfect matching, or $K$ has a $w_{r}$-balanced alternating cycle $C$. Since $K$ has a perfect matching, $C$ exists. Since $C$ is well-fitted to $K$, and $K$ is well-fitted to $G$, Lemma 22 (p. 38) implies $C$ is well-fitted to $G$. Then $C$ is a $w$-balanced alternating cycle of $G$. Therefore, $w$ is not unbalanced.

Given the preceding theorem, version 8 is equivalent to the following version.

Version 19. Does a given bipartite graph have a well-fitted $K_{3,3}$ bisubdivision? 


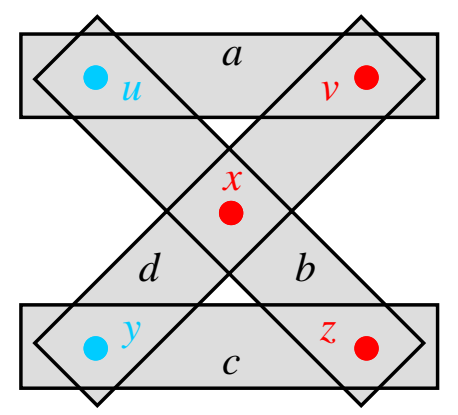

(a)

\section{$A(u, v) \wedge A(u, x, z) \wedge A(y, z) \wedge A(v, x, y)$

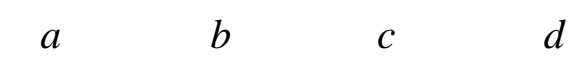

$A(u, v) \wedge A(u, x, z) \wedge A(y, z) \wedge A(v, x, y)$

(b)

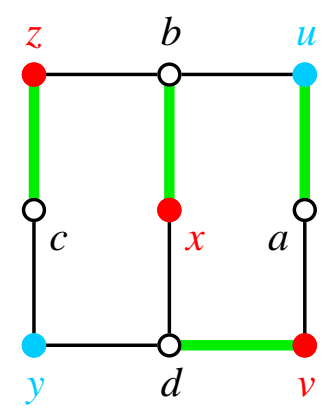

(c)

Figure 15: A hypergraph with a balanced 2-vertex colouring, a satisfiable conjunction of not-all-equal clauses, and a bipartite graph with a balanced 2-colouring of a colour class.

A hypergraph $H$ is an ordered pair $(V, E)$, where $V$ is a set and $E$ is a set of subsets of $V$. The elements of $V$ are called the vertices, and the elements of $E$ are called the hyperedges. A 2-vertex colouring of $H$ is a function from $V$ to a set of size two, say \{red, blue (F. 15a). A 2-vertex colouring is balanced if every hyperedge contains a red vertex and a blue vertex. $H$ has a system of distinct representatives if there exists an injective function $f: E(H) \rightarrow V(H)$ such that $f(e) \in e$ for every hyperedge $e$.

Suppose we have a truth assignment for the propositions $P_{1}, \ldots, P_{n}$. A not-all-equal clause $A\left(P_{1}, \ldots, P_{n}\right)$ is true if some $P_{i}$ is true and some $P_{j}$ is false (F.15b). A conjunction $F$ of not-all-equal clauses is satisfiable if there is a truth assignment for the set of propositions such that all not-all-equal clauses of $F$ are true. $F$ is matchable if there exists an injective function from its set of not-all-equal clauses to the set of propositions such that each clause $A\left(P_{1}, \ldots, P_{n}\right)$ get mapped into $\left\{P_{1}, \ldots, P_{n}\right\}$.

Let $G$ be a bipartite graph with colour classes $A$ and $B$. A 2-colouring of $B$ is a function from $B$ to a set of size two, say $\{$ red, blue\} (F. 15c). A 2-colouring of $B$ is balanced if every vertex in $A$ is adjacent to a red vertex and a blue vertex. $G$ is $A$-matchable if $G$ has a matching which saturates every vertex in $A$.

Version 20. Given a hypergraph $H$ with a system of distinct representatives, does $H$ have a balanced 2-vertex colouring?

Version 21. Is a matchable conjunction of not-all-equal clauses satisfiable?

Version 22. Let $G$ be a bipartite graph with colour classes $A$ and $B$ such that $G$ is $A$-matchable. Does $G$ have a balanced 2-colouring of $B$ ?

To motivate version 20, consider the following two observations. If $H$ is a hypergraph with no balanced 2-vertex colouring, and we keep removing hyperedges from $H$, we will eventually get a subhypergraph of $H$ with a balanced 2-vertex colouring. If $H$ is a hypergraph with a balanced 2-vertex colouring and at least one hyperedge, and we keep removing vertices from $H$, we will eventually get a subhypergraph of $H$ with no balanced 
2 -vertex colouring. Considering these observations one might wonder if a hypergraph has a balanced 2-vertex colouring if the number of vertices is "large" relative to the number of hyperedges. But this is trivially false: consider a hypergraph with hyperedges $\left\{v_{1}, v_{2}\right\}$, $\left\{v_{1}, v_{3}\right\},\left\{v_{2}, v_{3}\right\}$, and $\left\{v_{1}, v_{2}, v_{3}, \ldots, v_{10^{10}}\right\}$. To get away from such easy counterexamples, one might ask if a hypergraph has a balanced 2-vertex colouring if the number of vertices is "large" relative to the number of hyperedges, and the vertices are "equitably distributed" amongst the hyperedges. Version 20 asks such a question.

We will follow the work of Seymour [41] to show that versions 20, 21, and 22 are equivalent to version 17. We first need Theorem 13.

Let $G$ be a bipartite graph with colour classes $A$ and $B$ such that $G$ is $A$-matchable. Suppose $\emptyset \neq X \subset A$. We say $H=G[X \cup N(X)]$ is a supercondenser (F. 16a) if $|X|=|N(X)|$ and every alternating cycle of $H$ has length congruent to 2 modulo 4 .

Theorem 13 If $G$ is a bipartite graph with colour classes $A$ and $B$, and $G$ is $A$-matchable, then $G$ has a balanced 2-colouring of $B$ if and only if $G$ does not have a supercondenser.

Proof $(\Rightarrow)$. Suppose $G$ has a balanced 2-colouring. Let $X$ be a nonempty subset of $A$ such that $|X|=|N(X)|$. We will show $H=G[X \cup N(X)]$ is not a supercondenser (F. 16b). Since $G$ is $A$-matchable, $G$ has a matching that saturates all vertices in $A$. Then some subset of this matching is a perfect matching $M$ of $H$ because $|X|=|N(X)|$. Suppose $P$ is a path $b_{1} x_{1} \cdots b_{n} x_{n}$ of $H$ such that $\left\{x_{1}, \ldots, x_{n}\right\} \subset X,\left\{b_{1} x_{1}, \ldots, b_{n} x_{n}\right\} \subset M$, and $b_{i}$ and $b_{i+1}$ have different colours for $i=1, \ldots, n-1$. (Such a path exists because $X$ is nonempty.) Choose $P$ to have maximum length. We may assume $b_{n}$ is coloured red. Since the 2-colouring of $B$ is balanced, there exists a blue vertex $b$ adjacent to $x_{n}$. Then there exists an edge $b x$ in $M$. If $b$ is not on $P$, then $b_{1} x_{1} \cdots b_{n} x_{n} b x$ contradicts the maximality of $P$. Hence, $b$ is on $P$. Then $C=P\left[b, x_{n}\right]+x_{n} b$ is an $M$-alternating cycle. Furthermore, the length of $C$ is congruent to 0 modulo 4 because the vertices of $C$ in $B$ are alternately coloured red and blue. Hence, $H$ is not a supercondenser.

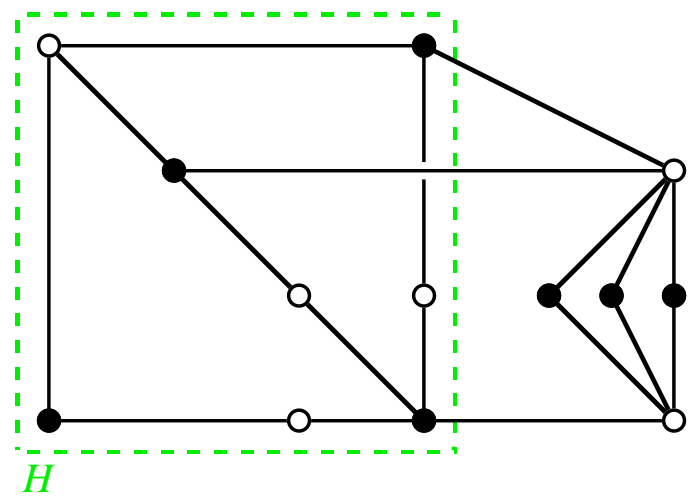

(a)

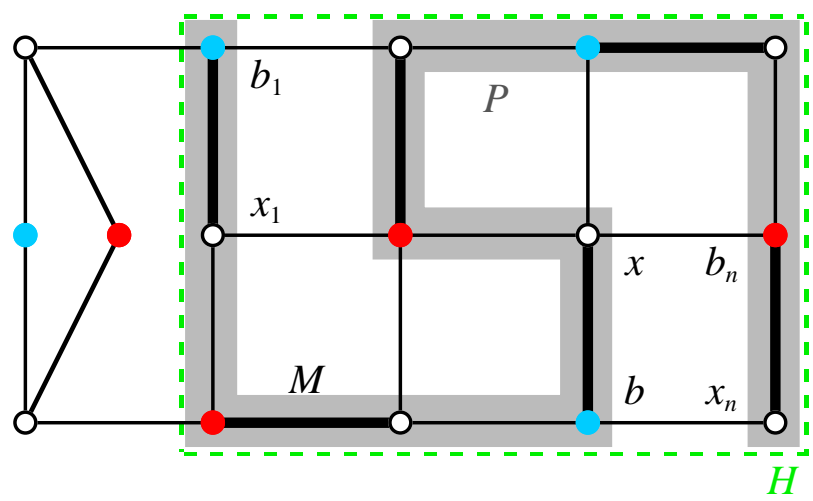

(b)

Figure 16: A supercondenser, and an example for the proof of Theorem 13. 
(a)

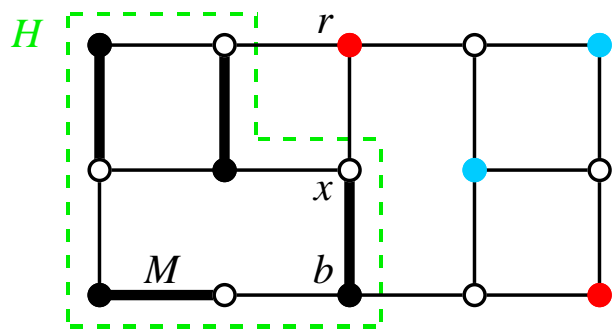

(c)

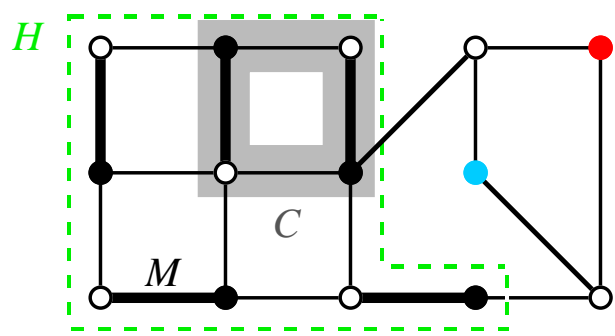

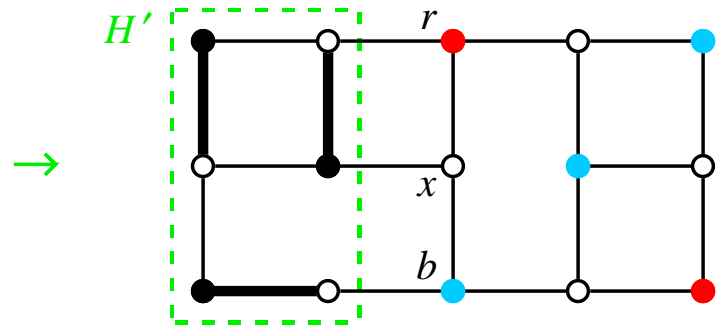

(b)

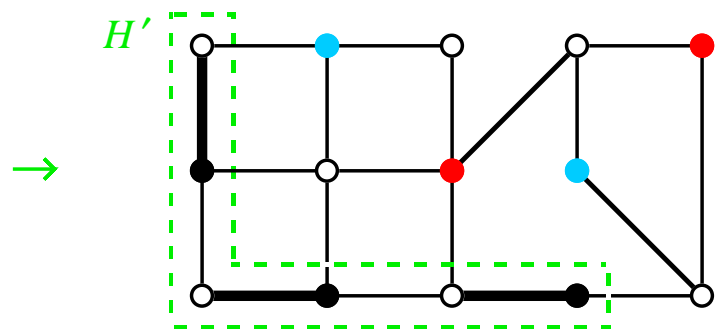

(d)

Figure 17: Examples for the proof of Theorem 13.

$(\Leftarrow)$. Suppose $G$ has no supercondenser, that is, suppose for every nonempty subset $X$ of $A$ such that $|X|=|N(X)|$, there is an alternating cycle of $G[X \cup N(X)]$ whose length is congruent to 0 modulo 4 .

Call a subgraph $H$ of $G$ uncoloured (F. 17ac) if $H$ has a perfect matching and $G-V(H)$ has a balanced 2-colouring of $B-V(H)$. It follows from the definition that $G$ has a balanced 2-colouring of $B$ if and only if the empty subgraph is uncoloured. First we need to show $G$ has an uncoloured subgraph. Since $G$ is $A$-matchable, it has a matching $M$ which saturates all the vertices in $A$. Then the subgraph of $G$ induced by the vertices saturated by $M$ is uncoloured. To complete the proof, we need to show that any nonempty uncoloured subgraph can be replaced by a smaller uncoloured subgraph.

Suppose $H$ is a nonempty uncoloured subgraph of $G$. Let $f$ be a balanced 2-colouring of $B-V(H)$ for $G-V(H)$, and let $X=A \cap V(H)$.

Suppose some $x$ in $X$ is adjacent to a vertex $r$ in $B-V(H)$ (F. 17a). Choose a perfect matching $M$ of $H$, and let $b$ be the vertex such that $x b \in M$. Let $H^{\prime}=H-\{x, b\}$ (F. 17b). We may assume $f(r)$ is red. Extend $f$ by colouring $b$ blue. Then we have a balanced 2-colouring of $B-V\left(H^{\prime}\right)$ for the $G-V\left(H^{\prime}\right)$. Furthermore, $M-\{x b\}$ is a perfect matching of $H^{\prime}$. Therefore, $H^{\prime}$ is an uncoloured subgraph which is smaller than $H$.

Suppose no vertex in $X$ is adjacent to a vertex in $B-V(H)$ (F. 17c). It follows that $|X|=|N(X)|$. Since $G$ has no supercondenser, $H=G[X \cup N(X)]$ has a perfect matching $M$, and an $M$-alternating cycle $C$ of whose length is congruent to 0 modulo 4 . Let $H^{\prime}=H-V(C)(\mathrm{F} .17 \mathrm{~d})$. Extend $f$ by alternately colouring the vertices in $B \cap V(C)$ red and blue. Then we have a balanced 2-colouring of $B-V\left(H^{\prime}\right)$ for the $G-V\left(H^{\prime}\right)$. Furthermore, $M-E(C)$ is a perfect matching of $H^{\prime}$. Therefore, $H^{\prime}$ is an uncoloured subgraph which is smaller than $H$. 
Every conjunction $F$ of not-all-equal clauses naturally corresponds to a hypergraph $H$, and a bipartite graph $G$ (F. 15). Furthermore, a truth assignment satisfying $F$ naturally corresponds to a balanced 2-vertex colouring of $H$, and a balanced 2-colouring of a colour class $B$ of $G$. Hence, determining if $H$ and $G$ have such colourings is equivalent to determining if there is a truth assignment satisfying $F$. Schaefer [39] proved these three problems are NP-complete. It is easy to see that $F$ is matchable if and only if $H$ has a system of distinct representatives, and if and only if $G$ is $A$-matchable for some colour class $A$. Thus, versions 20, 21, and 22 are equivalent. We will show versions 17 and 22 are equivalent with the aid of Theorem 13.

Suppose there exists a polynomial algorithm $\mathcal{A}_{17}$ for version 17 . Then $\mathcal{A}_{17}$ determines if a given bipartite graph $G$ has an alternating cycle $C$ whose length is divisible by 4 , but it can also be used to efficiently find $C$ if it exists. Suppose $\mathcal{A}_{17}$ tells us that $C$ exists. Call an edge $e$ critical if $G-e$ has no alternating cycle whose length is divisible by 4 . Applying $\mathcal{A}_{17}$ to $G-e$ determines if an edge $e$ is critical. If all edges of $G$ are critical, then we are done because every component $G$ is either $C$ or a $K_{2}$. Otherwise, we remove a noncritical edge $e$, and repeat the process for $G-e$. Eventually we find $C$.

Now suppose $\mathcal{A}_{17}$ exists, and we have an instance of version 22, that is, we have a bipartite graph $G$ with colour classes $A$ and $B$ such that $G$ is $A$-matchable, and we want to determine if $G$ has a balanced 2-colouring of $B$. We use the algorithm given on the previous page to find a minimal uncoloured subgraph $H$ of $G$. Here we need to apply $\mathcal{A}_{17}$ to uncoloured subgraphs of $G$ to determine if they have alternating cycles of length divisible by 4 , and to find such cycles when they exist. If $H$ is the empty graph, then $G$ has a balanced 2-colouring of $B$. If $H$ is nonempty, then it is a supercondenser; and so $G$ does not have a balanced 2-colouring of $B$ by Theorem 13 .

Suppose there exists a polynomial algorithm $\mathcal{A}_{22}$ for version 22 , and suppose we have an instance of version 17, that is, we want to determine if a bipartite graph $G$ has an alternating cycle whose length is divisible by 4 . A bipartite graph has such a cycle if and only if it has a perfect matching, and some maximal 1-extendible subgraph has such a cycle. Hence, we may assume $G$ is 1-extendible. Let $A$ and $B$ be the colour classes of $G$. Since $G$ is 1-extendible, it is also $A$-matchable. We use $\mathcal{A}_{22}$ to determine if $G$ has a balanced 2-colouring of $B$. By Theorem 13 we have also determined if $G$ has a supercondenser. Since $G$ is 1-extendible, Theorem 3 implies that the only nonempty subset $X$ of $A$ such that $|X|=|N(X)|$ is $A$ itself. Thus, $G$ is the only possible supercondenser. Hence, we have determined if $G$ is a supercondenser. Since $G$ is a supercondenser if and only if every alternating cycle has length congruent to 2 modulo 4 , we have determined if $G$ has an alternating cycle whose length is divisible by 4 .

We prove the Fano plane (F. 4, p. 8) has no balanced 2-vertex colouring as follows. Suppose we colour each vertex with red or blue. We may assume there are at most three red vertices. If there are at most two red vertices, then at most five hyperedges contain a red vertex. If there are three red vertices, and they in some hyperedge, then that hyperedge contains no blue vertex. If there are three red vertices, and no hyperedge contains all three, then exactly six hyperedges contain a red vertex. For all possibilities, there is a monochromatic hyperedge. Hence, the 2-vertex colouring is not balanced. 
Alternatively, we can argue as follows. It is routine to show the Heawood graph $H_{14}$ (F. 4) has no alternating cycle whose length is divisible by 4. Then Theorem 13 implies $H_{14}$ has no balanced 2-colouring of one of its colour class. This in turn implies the corresponding hypergraph, the Fano plane, has no balanced 2-vertex colouring.

Consider the game of tic-tac-toe. Let $H$ be the hypergraph whose vertices are the nine squares and whose hyperedges are the eight winning triples (2 diagonals, 3 columns, and 3 rows). Let " $\mathrm{x}$ " and "o" be our two colours. Neither player wins a game if and only if each winning triple has a square with an " $\mathrm{x}$ " and a square with an "o". Hence, a game is a draw if and only if the 2-vertex colouring is balanced. A version of tic-tac-toe can be played on any hypergraph. For all versions, a game is a draw if and only if the resulting 2-vertex colouring is balanced. When the game is played using the Fano plane (tic-tac-Fano), one player always wins because the Fano plane has no balanced 2-vertex colouring.

Version 23. Does a given bipartite graph have a Pfaffian orientation?

Pfaffian orientations of graphs are defined on page 14 .

Let $G$ be a bipartite graph with colour classes $R$ and $C$. A $\{-1,1\}$-edge weighting $w$ of $G$ corresponds to an orientation $\vec{G}$ if for every edge $r c$ of $G$, where $r \in R$ and $c \in C$, $w(r c)=1$ if and only if $r c \in A(\vec{G})$ (F. 18). Thus, there is a natural bijection between $\{-1,1\}$-edge weightings and orientations of $G$.

unbalanced $\{-1,1\}$-edge

weighting of $G$

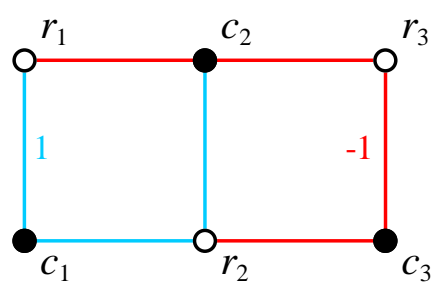

$(D, m)$

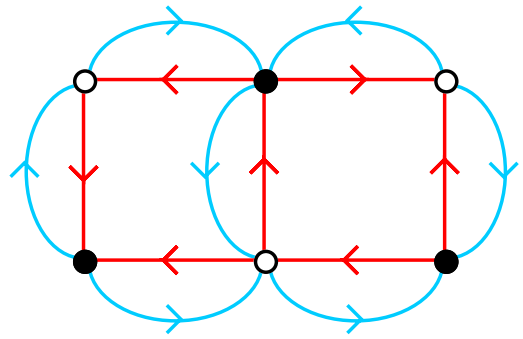

Pfaffian orientation $\vec{G}$

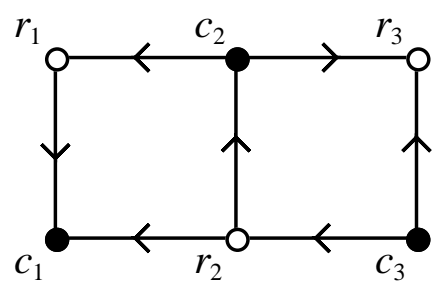

$A=\left[\begin{array}{ccc}1 & -1 & 0 \\ 1 & 1 & -1 \\ 0 & -1 & -1\end{array}\right]$

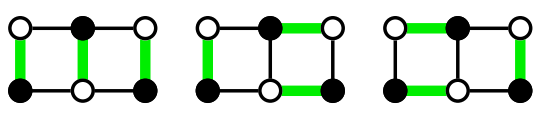

$\operatorname{det}(A)=a_{11} a_{22} a_{33}-a_{11} a_{23} a_{32}-a_{12} a_{21} a_{33}=-3$

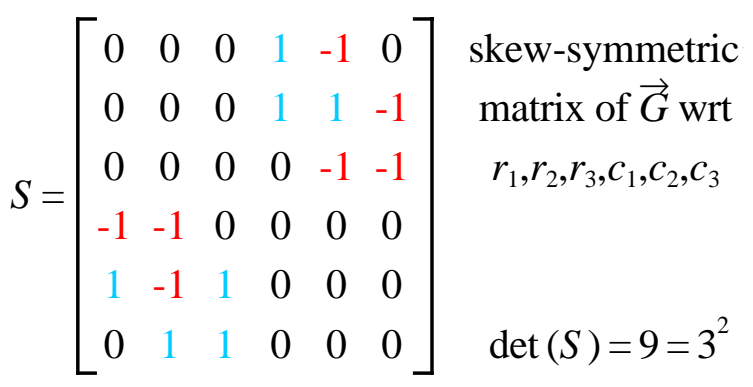

Figure 18: A Pfaffian orientation of a bipartite graph. 
The following result implies that version 23 is equivalent to version 8 .

Theorem 14 Suppose $w$ is a $\{-1,1\}$-edge weighting of a bipartite graph $G$ with colour classes $R$ and $C$. Let $\vec{G}$ be the corresponding orientation. If $G$ has a perfect matching, then $w$ is unbalanced if and only if $\vec{G}$ is Pfaffian.

Proof. We first make some useful definitions. Let $D$ be the symmetric digraph obtained from $G$ by replacing each edge $r c$ by the arcs $r c$ and $c r$ (F. 18). Define a $\{-1,1\}$-arc weighting $m$ of $D$ by $m(r c)=-w(r c)$ and $m(c r)=w(r c)$, for every edge $r c$ of $G$, where $r \in R$ and $c \in C$. A cycle of $G$ is Pfaffian in $\vec{G}$ if it has an odd number of arcs going in each direction.

Suppose $C=r_{0} c_{0} r_{1} c_{1} \cdots r_{k-1} c_{k-1} r_{0}$ is an alternating cycle of $G$, where $r_{i} \in R$ and $c_{i} \in C$ for $i=0, \ldots, k-1$, and let $m(C)=m\left(r_{0} c_{0}\right) m\left(c_{0} r_{1}\right) \cdots m\left(r_{k-1} c_{k-1}\right) m\left(c_{k-1} r_{0}\right)$. For $i=0, \ldots, k-1$,

$$
\begin{aligned}
& r_{i} c_{i} \in A(\vec{G}) \Leftrightarrow w\left(r_{i} c_{i}\right)=1 \Leftrightarrow m\left(r_{i} c_{i}\right)=-1, \quad \text { and } \\
& c_{i} r_{i+1} \in A(\vec{G}) \Leftrightarrow w\left(r_{i+1} c_{i}\right)=-1 \Leftrightarrow m\left(c_{i} r_{i+1}\right)=-1 .
\end{aligned}
$$

Thus, an edge $u v$ of $C$ is oriented in the forward direction around $C$ if and only if $m(u v)=-1$. It follows that $C$ is Pfaffian in $\vec{G}$ if and only if $m(C)=-1$. Furthermore,

$$
\begin{aligned}
m(C) & =\left[m\left(r_{0} c_{0}\right) m\left(r_{1} c_{1}\right) \cdots m\left(r_{k-1} c_{k-1}\right)\right] \cdot\left[m\left(c_{0} r_{1}\right) m\left(c_{1} r_{2}\right) \cdots m\left(c_{k-1} r_{0}\right)\right] \\
& =\left[(-1)^{k} w\left(r_{0} c_{0}\right) w\left(r_{1} c_{1}\right) \cdots w\left(r_{k-1} c_{k-1}\right)\right] \cdot\left[w\left(r_{1} c_{0}\right) w\left(r_{2} c_{1}\right) \cdots w\left(r_{0} c_{k-1}\right)\right] \\
& =(-1)^{k} \cdot w(C) .
\end{aligned}
$$

Hence, $m(C)=-1 \Leftrightarrow w(C)=(-1)^{k-1} \Leftrightarrow C$ is $w$-unbalanced.

Therefore, $C$ is Pfaffian in $\vec{G}$ if and only if $C$ is $w$-unbalanced. It follows that $\vec{G}$ is a Pfaffian orientation if and only if $w$ is unbalanced.

Theorem 14 gives a natural bijection between unbalanced $\{-1,1\}$-edge weightings and Pfaffian orientations of a bipartite graph. When such a weighting and orientation exist, we can efficiently calculate the number of perfect matchings using the next result.

Corollary 15 Suppose $w$ is an unbalanced $\{-1,1\}$-edge weighting of a bipartite graph $G$. If $A$ is a matrix of $(G, w)$, then $|\operatorname{det}(A)|$ is the number of perfect matchings of $G$.

Proof. (F. 18). Since $w$ is unbalanced, Theorem 4 implies the terms of $\operatorname{det}(A)$ are unbalanced. Hence, all nonzero terms of $\operatorname{det}(A)$ have the same sign. Furthermore, all nonzero terms of $\operatorname{det}(A)$ have absolute value 1 because $A$ is a $\{-1,0,1\}$-matrix. It follows that $|\operatorname{det}(A)|$ is the number of nonzero terms of $\operatorname{det}(A)$. Since there is a natural bijection between the nonzero terms of $\operatorname{det}(A)$ and the perfect matchings of $G,|\operatorname{det}(A)|$ is also the number of perfect matchings of $G$. 
This result can be generalized to all (bipartite or nonbipartite) graphs with Pfaffian orientations. To explain how this is done, we first need to define a new type of matrix.

Suppose $\vec{G}$ is an orientation of a graph $G$. Let $v_{1}, \ldots, v_{n}$ be an ordering of $V(G)$. The skew-symmetric matrix of $\vec{G}$ with respect to $v_{1}, \ldots, v_{n}$ is the $n \times n$ matrix $S=\left[s_{i j}\right]$ such that

$$
s_{i j}=\left\{\begin{array}{cl}
1 & \text { if } v_{i} v_{j} \text { is an } \operatorname{arc} \text { of } \vec{G}, \\
-1 & \text { if } v_{j} v_{i} \text { is an } \operatorname{arc} \text { of } \vec{G}, \text { and } \\
0 & \text { otherwise. }
\end{array}\right.
$$

for $i, j=1, \ldots, n$.

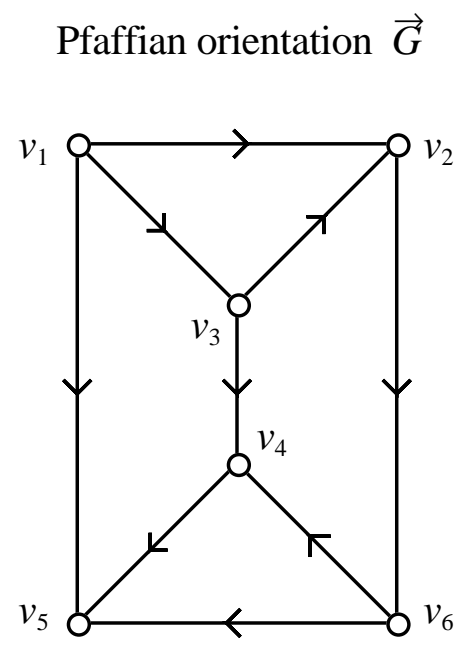

$$
\begin{gathered}
\underset{\text { skew-symmetric matrix of }}{\rightarrow} \text { wrt } v_{1}, v_{2}, v_{3}, v_{4}, v_{5}, v_{6} \\
S=\left[\begin{array}{cccccc}
0 & 1 & 1 & 0 & 1 & 0 \\
-1 & 0 & -1 & 0 & 0 & 1 \\
-1 & 1 & 0 & 1 & 0 & 0 \\
0 & 0 & -1 & 0 & 1 & -1 \\
-1 & 0 & 0 & -1 & 0 & -1 \\
0 & -1 & 0 & 1 & 1 & 0
\end{array}\right] \\
\operatorname{det}(S)=16=4^{2}
\end{gathered}
$$
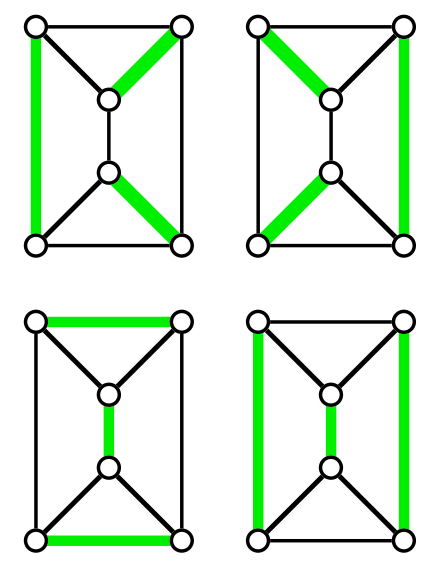

Figure 19: A Pfaffian orientation of a nonbipartite graph.

Theorem 16 (Kasteleyn [16]) Let $m$ be the number of perfect matchings of a graph $G$. Suppose $S$ is a skew-symmetric matrix of an orientation $\vec{G}$ of $G$ (Figures 18 and 19). If $\vec{G}$ is Pfaffian, then $m^{2}=\operatorname{det}(S)$.

Corollary 15 can be derived from Theorem 16. Suppose $w$ is an unbalanced edge weighting of a bipartite graph $G$ with row vertices $r_{1}, \ldots, r_{n}$ and column vertices $c_{1}, \ldots, c_{n}$. Let $A$ and $\vec{G}$ be the matrix and Pfaffian orientation, respectively, corresponding to $(G, w)$. Then the skew-symmetric matrix of $\vec{G}$ with respect to $r_{1}, \ldots, r_{n}, c_{1}, \ldots, c_{n}$ is

$$
S=\left[\begin{array}{c|c}
0 & A \\
\hline-A^{t} & 0
\end{array}\right]
$$

Now Theorem 16 implies $m^{2}=\operatorname{det}(S)=[\operatorname{det}(A)]^{2}$. Hence, $m=|\operatorname{det}(A)|$. 


\section{Braces}

This section contains some results about braces needed in the proof of the Main Theorem. In particular, we state some results from [31] on generating braces.

The first result is a routine corollary of Theorem 3 .

Corollary 17 If $x$ and $y$ are in different colour classes of a brace $H$, then $H-\{x, y\}$ is 1-extendible.

Corollary 18 (Plummer [34]) For every positive integer $k$, every $k$-extendible graph is $(k+1)$-connected and has minimum degree at least $k+1$.

Let $H_{10}$ be the 4-cycle sum of three $K_{3,3}$ graphs at a 4-cycle $C$ such that no edge of $C$ is in $E\left(H_{10}\right)$.

Lemma 19 Let $n \geq 3$, and let $G, G_{1}, \ldots, G_{n}$ be bipartite graphs such that $G$ is not an $H_{10}$ and $\nu\left(G_{i}\right) \geq 6$ for $i=1, \ldots, n$. Suppose $G$ is a 4 -cycle sum of $G_{1}, \ldots, G_{n}$ at $C=a b c d a$. Then $G$ is a brace if and only if $G_{1}, \ldots, G_{n}$ are braces.

Proof $(\Rightarrow)$. Suppose $G$ is a brace. Then statement (b) of Theorem 3 implies $G+E(C)$ is also a brace. Suppose $1 \leq i \leq n$. By assumption, $\nu\left(G_{i}\right) \geq 6$. Since $G_{i}$ has the 4-cycle $C$, it has two nonadjacent edges. Furthermore, $G_{i}$ is connected because $G+E(C)$ is connected. Since $G$ is a brace, it has a perfect matching containing $a b$ and $c d$; and so $G_{i}-V(C)$ has the same number of vertices in each colour class.

Suppose $e$ and $f$ are nonadjacent edges of $G_{i}$. The brace $G+E(C)$ has a perfect matching $M$ containing $e$ and $f$. Since $G_{i}-V(C)$ has the same number of vertices in each colour class, $M \cap E\left(G_{i}\right)$ is a matching of $G_{i}$ which saturates all vertices except an even number of vertices on $C$, and the number of unsaturated vertices in each colour class is the same. Hence, the union of $M \cap E\left(G_{i}\right)$ and an appropriate subset of $E(C)$ is a perfect matching of $G_{i}$ containing $e$ and $f$. Therefore, $G_{i}$ is a brace.

$(\Leftarrow)$. Suppose $G_{1}, \ldots, G_{n}$ are braces. If $n=3$ and $G_{1}, G_{2}$, and $G_{3}$ are $K_{3,3}$ graphs, then it is easy to show $G$ is a brace. (Remember, $G$ is not an $H_{10}$.) Hence, we will assume $G$ is not a 4-cycle sum of three $K_{3,3}$ graphs. If we can prove $G-E(C)$ is a brace, then $G$ is also a brace. Hence, we can also assume $G$ does not use any edges of $C$.

First we show $G$ is connected. Suppose $1 \leq i \leq n$. By Corollary 18 we know $G_{i}$ is 3-connected. Then $G_{i}-\{a, c\}$ is connected, and $a$ and $c$ are adjacent to vertices in $V\left(G_{i}\right)-V(C)$. Hence, $G_{i}-E(C)$ is connected. Therefore, $G=\sum_{i=1}^{n}\left[G_{i}-E(C)\right]$ is connected.

Second we make the trivial observations that $\nu(G) \geq \nu\left(G_{1}\right) \geq 6$ and $G$ has two nonadjacent edges.

Third we show that for any nonadjacent edges $e$ and $f$ of $G$, there is a perfect matching of $G$ containing $e$ and $f$. We may assume $e \in E\left(G_{1}\right)-E(C)$ and $f \in E\left(G_{1}+G_{2}\right)-E(C)$. There are two cases, where the second case has three subcases. 
Suppose $e$ and $f$ are in $E\left(G_{1}\right)-E(C)$. Since $G_{1}$ is a brace, $e$ and $f$ are contained in some perfect matching $M_{1}$ of $G_{1}$. We may assume $M_{1} \cap E(C) \subset\{a b, c d\}$. Next choose a perfect matching $M_{2}$ for $G_{2}$ using the fact that $G_{2}$ is a brace; but the choice of $M_{2}$ depends on whether $M_{1}$ contains $a b$. If $a b \in M_{1}$, choose $M_{2}$ to include $c d$ and some edge $a y$, where $y$ is not on $C$. If $a b \notin M_{1}$, choose $M_{2}$ to include $a b$ and $c d$. For both possibilities we get a perfect matching $M_{2}$ of $G_{2}$ containing $c d$ such that $a b \in M_{2}$ if and only if $a b \notin M_{1}$. Similarly, we can choose a perfect matching $M_{3}$ of $G_{3}$ containing $a b$ such that $c d \in M_{3}$ if and only if $c d \notin M_{1}$. If $n \geq 4$, then for every $i$ in $\{4, \ldots, n\}$, choose a perfect matching $M_{i}$ of $G_{i}$ which includes $a b$ and $c d$. Then $\left(\cup_{i=1}^{n} M_{i}\right)-\{a b, c d\}$ is a perfect matching of $G$ which includes $e$ and $f$.

Suppose $e \in E\left(G_{1}\right)-E(C)$ and $f \in E\left(G_{2}\right)-E(C)$. For this case there are three subcases.

Suppose $e$ or $f$ is incident with $C$. We may assume $e$ is incident with $a$. Then we may assume that if $f$ is incident with a vertex of $C$, then it is incident with $c$ or $d$. Choose a perfect matching $M_{1}$ for $G_{1}$ such that $\{e, c d\} \subset M_{1}$, and a perfect matching $M_{2}$ for $G_{2}$ such that $\{f, a b\} \subset M_{2}$. Then choose a perfect matching $M_{3}$ for $G_{3}$ containing $a b$ such that $c d \in M_{3}$ if and only if $c d \notin M_{2}$. If $n \geq 4$, then for every $i$ in $\{4, \ldots, n\}$, choose a perfect matching $M_{i}$ of $G_{i}$ containing $a b$ and $c d$. Then $\left(\cup_{i=1}^{n} M_{i}\right)-\{a b, c d\}$ is a perfect matching of $G$ which includes $e$ and $f$.

Suppose $e$ and $f$ are not incident with $C$, and $G_{1}$ or $G_{2}$ is not a $K_{3,3}$. We may assume $G_{2}$ is not a $K_{3,3}$. Then there exists an edge $g$ in $E\left(G_{2}\right)-E(C)$ incident with $C$ such that $f$ and $g$ are nonadjacent. We may assume $g$ is incident with $c$. Choose a perfect matching $M_{1}$ for $G_{1}$ such that $\{e, a b\} \subset M_{1}$. Next choose a perfect matching $M_{2}$ for $G_{2}$; but the choice depends on whether $c d$ is in $M_{1}$. If $c d \notin M_{1}$, choose $M_{2}$ to contain $f$ and $c d$. If $c d \in M_{1}$, choose $M_{2}$ to contain $f$ and $g$. When $a b, c d \in M_{1}$ and $f, g \in M_{2}$, there is symmetry between $b$ and $d$ because we can shift $M_{1}$ on $C$; and so we may assume $M_{2}$ contains an edge incident with $d$ but not on $C$. Then choose a perfect matching $M_{3}$ for $G_{3}$ containing $c d$ such that $a b \in M_{3}$ if and only if $a b \notin M_{2}$. If $n \geq 4$, then for every $i$ in $\{4, \ldots, n\}$, choose a perfect matching $M_{i}$ of $G_{i}$ containing $a b$ and $c d$. Then $\left(\cup_{i=1}^{n} M_{i}\right)-\{a b, c d\}$ is a perfect matching of $G$ which includes $e$ and $f$.

Suppose $e$ and $f$ are not incident with $C$, and $G_{1} \cong K_{3,3} \cong G_{2}$. Let $M_{1}=\{e, a b, c d\}$ and $M_{2}=\{f, a b, c d\}$. Suppose $G_{3}$ is not a $K_{3,3}$. Then there exist nonadjacent edges ay and $c z$ incident with $C$. Choose a perfect matching $M_{3}$ for $G_{3}$ such that $\{a y, c z\} \subset M_{3}$. Then $M_{3} \cap E(C)=\emptyset$. If $n \geq 4$, then for every $i$ in $\{4, \ldots, n\}$, choose a perfect matching $M_{i}$ of $G_{i}$ containing $a b$ and $c d$. Then $\left(\cup_{i=1}^{n} M_{i}\right)-\{a b, c d\}$ is a perfect matching of $G$ which includes $e$ and $f$. Otherwise, we may assume $G_{3}, \ldots, G_{n}$ are $K_{3,3}$ graphs. Since we are assuming $G$ is not a 4-cycle sum of three $K_{3,3}$ graphs, $n \geq 4$. Choose a perfect matching $M_{3}$ for $G_{3}$ such that $M_{3} \cap E(C)=\{a b\}$, and a perfect matching $M_{4}$ for $G_{4}$ such that $M_{4} \cap E(C)=\{c d\}$. If $n \geq 5$, then for every $i$ in $\{5, \ldots, n\}$, choose a perfect matching $M_{i}$ of $G_{i}$ containing $a b$ and $c d$. Then $\left(\cup_{i=1}^{n} M_{i}\right)-\{a b, c d\}$ is a perfect matching of $G$ which includes $e$ and $f$.

We have shown $G$ is connected, $G$ has two nonadjacent edges, $\nu(G) \geq 6$, and any two nonadjacent edges of $G$ are in a perfect matching of $G$. Therefore, $G$ is a brace. 


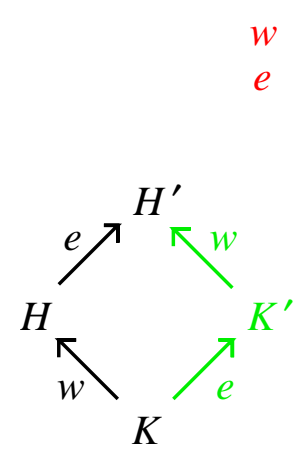

(a)

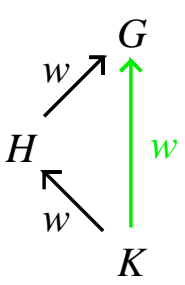

(b)

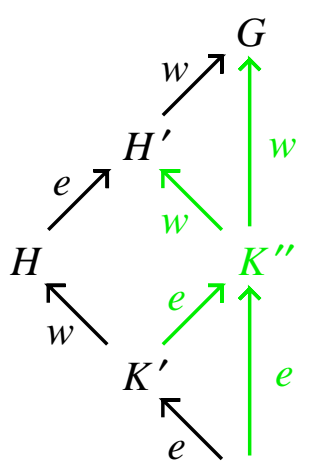

(c) $a$ augmentation

$p$ predecessor

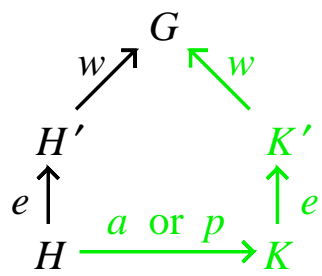

(d)

Figure 20: Lemmas 21, 22, 23, and 25.

The next result is needed in $[\mathbf{3 0}, \mathbf{p}$. 57]. Its proof follows the proof of Theorem 19 in the "easy" direction.

Lemma 20 Let $G_{1}$ and $G_{2}$ be bipartite graphs such that $G_{1} \cap G_{2}$ is a 4-cycle, and $G_{1}$ and $G_{2}$ are proper subgraphs of $G_{1} \cup G_{2}$. If $G_{1} \cup G_{2}$ is a brace, then $G_{1}$ and $G_{2}$ are braces.

Let $H$ be a bipartite graph with $\delta \geq 2$. Suppose $x$ is in $V(H)$, and $\left\{Z_{1}, Z_{2}\right\}$ is a partition of $N(x)$. Let $H^{\prime}=(H-x)+x_{1} v x_{2}+\left\{x_{1} z \mid z \in Z_{1}\right\}+\left\{x_{2} z \mid z \in Z_{2}\right\}$, where $x_{1}$, $v$, and $x_{2}$ are new vertices. We say $H^{\prime}$ is obtained from $H$ by expanding $x$ (to $x_{1} v x_{2}$ ). If $H^{\prime \prime}$ is obtained from $H$ via a sequence of vertex expansions, then we say $H^{\prime \prime}$ is an expansion of $H$. Note that a bisubdivision is an expansion, and that for a graph with maximum degree three, an expansion is a bisubdivision.

The next two lemmas are from $[31$, p. 154], and Lemma 23 follows easily from them.

Lemma 21 (F. 20a) If $K$ is a well-fitted subgraph of a bipartite graph $H$, and $H^{\prime}$ is an expansion of $H$, then $K$ has an expansion $K^{\prime}$ which is well-fitted to $H^{\prime}$.

Lemma 22 (F. 20b) Let $K, H$, and $G$ be bipartite graphs. If $K$ is well-fitted to $H$, and $H$ is well-fitted to $G$, then $K$ is well-fitted to $G$.

Lemma 23 (F. 20c) Let $K, H$, and $G$ be bipartite graphs. If $K$ has an expansion which is well-fitted to $H$, and $H$ has an expansion which is well-fitted to $G$, then $K$ has an expansion which is well-fitted to $G$.

Next we describe a method of generating braces. Four operations are needed (F. 21). Let $H$ be a brace with colour classes $A$ and $B$. A bipartite graph obtained from $H$ by adding a new edge is called a type 1 augmentation of $H$. Suppose $x$ and $w$ are in $A$, and $H^{\prime}$ is obtained from $H$ by expanding $x$ to $x_{1} v x_{2}$. If $\delta \geq 3$ for $H^{\prime}+w v$, then we say it is a type 2 augmentation of $H$. (We can also get a type 2 augmentation starting with two vertices in $B$.) 

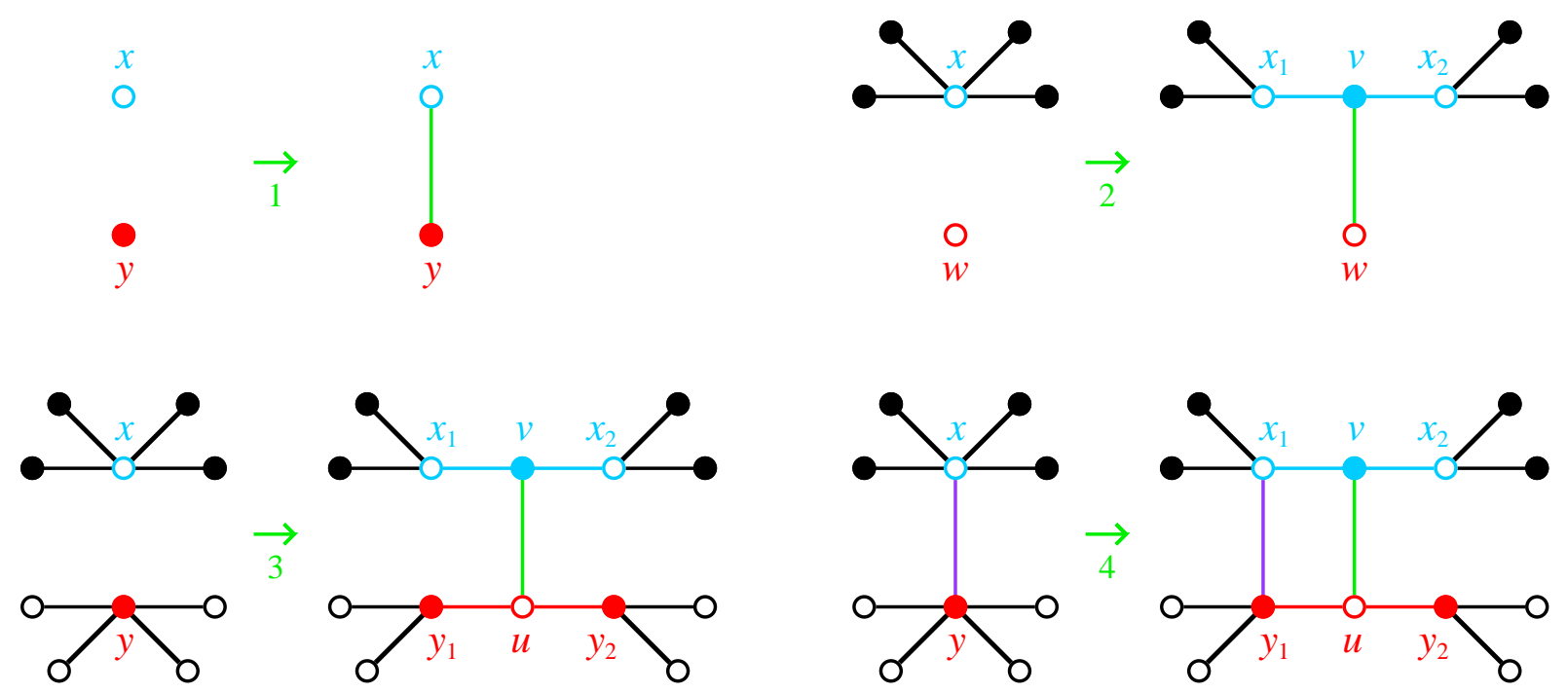

Figure 21: The four types of augmentations.

Suppose $x \in A$ and $y \in B$, and $H^{\prime \prime}$ is a graph obtained from $H$ by expanding $x$ to $x_{1} v x_{2}$ and expanding $y$ to $y_{1} u y_{2}$. If $\delta \geq 3$ for $H^{\prime \prime}+u v$, then we say it is a type 3 augmentation of $H$ when $x y \notin E(H)$, and a type 4 augmentation of $H$ when $x y \in E(H)$.

If $G$ is a type $i$ augmentation of $H$ for some $i$ in $\{1,2,3,4\}$, then we say $G$ is an augmentation of $H$, or an $H$ augmentation. When using augmentations we will adopt the notation of Figure 21. In particular, for a type 4 augmentation, we will assume $x_{1} y_{1}$ is the unique edge with one end in $\left\{x_{1}, x_{2}\right\}$ and one end in $\left\{y_{1}, y_{2}\right\}$.

Suppose $G$ is a type 2, 3, or 4 augmentation $H$, and $x$ is expanded to $x_{1} v x_{2}$ using the partition $\left\{Z_{1}, Z_{2}\right\}$ of $N(x)$. An $x_{i}$-edge is an edge $x z$ of $H$ such that $x_{i} z$ is an edge of $G$ for $i=1,2$. For a type 4 augmentation, this definition carefully excludes $x y$ from the set of $x_{1}$-edges. Since $\left\{Z_{1}, Z_{2}\right\}$ is a partition, no edge of $H$ is both an $x_{1}$-edge and an $x_{2}$-edge. By definition $G$ has minimum degree 3 ; and so there are minimum numbers of $x_{1}$-edges and $x_{2}$-edges. For type 2 and 3 augmentations, there are at least two $x_{i}$-edges for $i=1,2$. For a type 4 augmentation, $x_{1} y_{1}$ is an edge of $G$ by assumption; and so there is at least one $x_{1}$-edge, and there are at least two $x_{2}$-edges. There are similar definitions and comments for $y_{1}$-edges and $y_{2}$-edges.

Ladders, Möbius ladders, and biwheels are defined in Figure 22. Let $\mathcal{B}$ be the union of these three infinite sequences of bipartite graphs. Note that $M_{6}=K_{3,3}$. Let $\mathcal{B}_{2}$ be the set of bipartite graphs generated from the graphs in $\mathcal{B}$ using augmentations. To make the definition of $\mathcal{B}_{2}$ precise (F. 5, p. 9), $G$ is in $\mathcal{B}_{2}$ if and only if there exists a sequence $G_{0}, G_{1}, \ldots, G_{n}$ of bipartite graphs such that $G_{0} \in \mathcal{B}, G_{i+1}$ is a $G_{i}$ augmentation for $i=0,1, \ldots, n-1$, and $G=G_{n}$.

The next theorem is the principal result from $[31$, p. 127], and it is crucial for the proof of the Main Theorem of this paper.

Theorem $24 \mathcal{B}_{2}$ is the set of all braces. 
Define $B_{8}$ to be $L_{8}$, and $B_{8}^{+}$to be $L_{8}+x y$, where $x$ and $y$ are nonadjacent vertices of $L_{8}$ in different colour classes. For every even integer $r \geq 10$, define $B_{r}^{+}$to be $B_{r}+x y$, where $x$ and $y$ are the two vertices of $B_{r}$ with degree at least 4 . Let $\mathcal{B}^{+}=\mathcal{B} \cup\left\{B_{8}^{+}, B_{10}^{+}, \ldots\right\}$. For all integers $r, s \geq 8$ such that $r$ is even and $s$ is divisible by 4 , we say that $B_{r}, B_{r}^{+}$, $L_{s}$, and $M_{s-2}$ are the predecessors of $B_{r+2}, B_{r+2}^{+}, L_{s+4}$, and $M_{s+2}$, respectively.

The following result from $[31$, p. 162] will be needed once.

Lemma 25 (F. 20d) If $G$ and $H$ are braces, and some $H$ expansion $H^{\prime}$ is a well-fitted proper subgraph of $G$, then there exists a brace $K$ such that some $K$ expansion $K^{\prime}$ is well-fitted to $G$ and one of the following statements holds.

- $K$ is an $H$ augmentation.

- $\{H, K\} \subset \mathcal{B}^{+}$and $H$ is the predecessor of $K$.
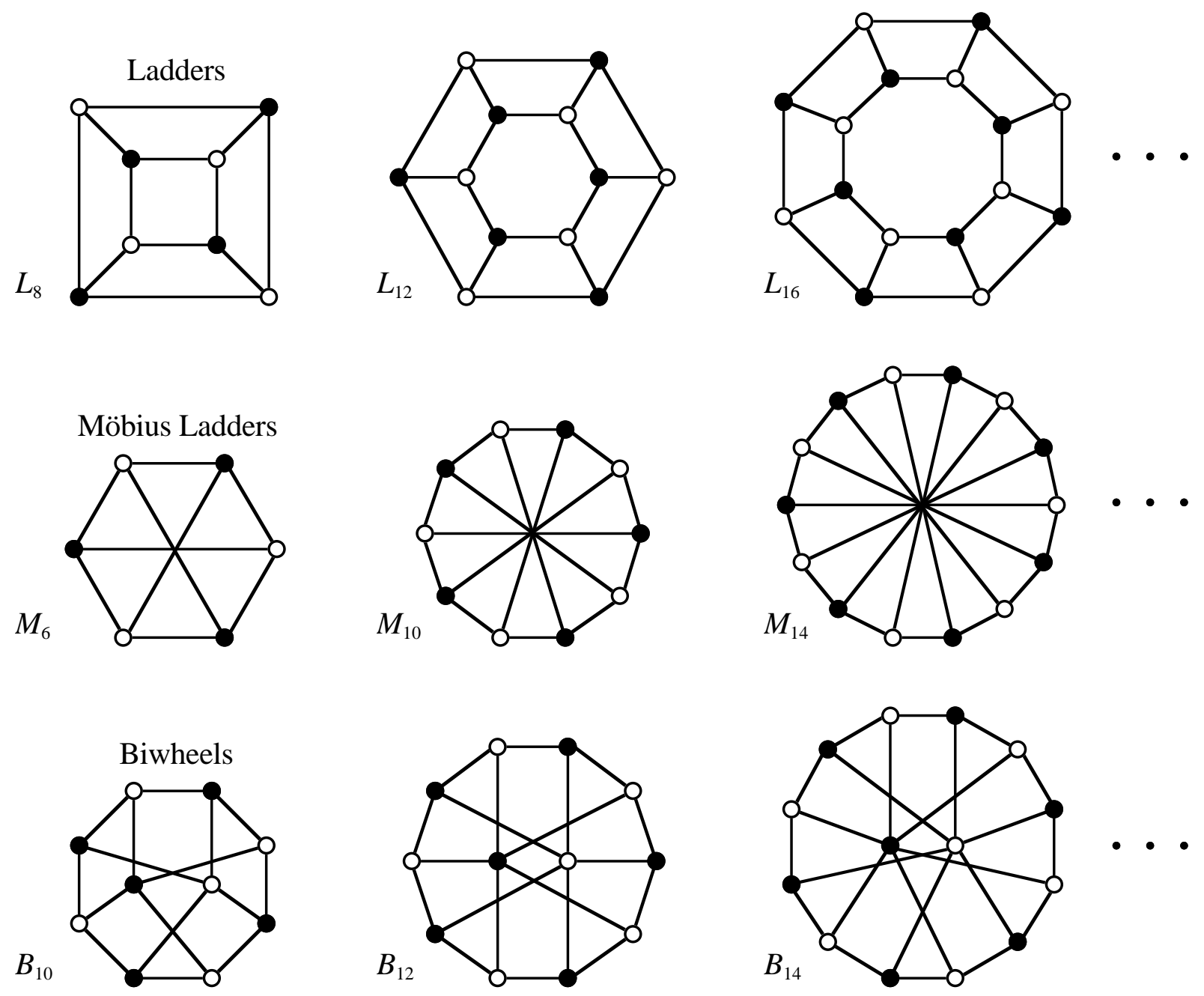

Figure 22: The base set $\mathcal{B}$. 


\section{Ear Paths}

In this section we provide some technical lemmas needed in the proof of the Main Theorem.

We will generally use $A$ and $B$ to denote the colour classes of a bipartite graph. In the figures the light vertices are in $A$ and the dark vertices are in $B$. If $P$ is a path with an end $x$ in $A$ and an end $y$ in $B$, then we will designate $x$ as the origin and $y$ as the terminus of $P$.

Let $M$ be a perfect matching of a bipartite graph $H$. If $P=x_{1} y_{1} \cdots x_{n} y_{n}$ is a path of $H$ such that $\left\{y_{i} x_{i+1} \mid 1 \leq i \leq n-1\right\} \subset M$, then we say $P$ is an $M$-ear path. (Note that $n=1$ is possible, in which case no edge in $M$ is on $P$.) Suppose $K$ is a subgraph of $H$ which is well-fitted to $M$. We say $P$ is an $M$-ear path of $K$ if its ends are on $K$, but none of its edges or intermediate vertices are on $K$. Suppose $P_{1}, \ldots, P_{r}$ are paths of $H$, where $r \geq 1$. We say $P_{1}, \ldots, P_{r}$ are ear paths of $K$ if there exists a perfect matching $M^{\prime}$ of $H$ such that $K$ is well-fitted to $M^{\prime}$, and $P_{1}, \ldots, P_{r}$ are all $M^{\prime}$-ear paths of $K$. (It follows that $K+P_{1}+\cdots+P_{r}$ is well-fitted to $M^{\prime}$.)

Let $G$ be a bipartite graph, and let $P$ be a path of odd length with both ends on $G$, but no edges or intermediate vertices on $G$. We say $G+P$ is obtained from $G$ by ear path addition. The following result has a routine proof.

Lemma 26 Let $K$ be a well-fitted subgraph of a brace $H$. Suppose $K$ can be obtained from an even cycle via a sequence of ear path additions. Then $K$ is 1-extendible. Suppose $P, Q$, and $R$ are internally disjoint odd length 2-paths of $K . K-P$ is well-fitted to $H$. If $P$ and $Q$ have a unique common end, then $K-(P+Q)$ is well-fitted to $H$. If $P$ and $Q$ are disjoint and $P+Q+R$ is a path, then $K-(P+R)$ is well-fitted to $H$.

Let $K$ be a well-fitted subgraph of a bipartite graph $H$. Suppose we have constructed ear paths $P$ and $Q$ of $K$ such that the ends of $P$ are distinct from the ends of $Q$. We would like $P \cap Q$ to be an "uncomplicated" graph. If $P \cap Q$ is "complicated", then the next lemma allows us to replace $P$ and $Q$ by ear paths $P^{\prime}$ and $Q^{\prime}$ of $K$ such that $P^{\prime} \cap Q^{\prime}$ is a path or the empty graph, $P^{\prime}$ has the same first and last edges as $P$, and $Q^{\prime}$ has the same first and last edges as $Q$. The lemma is proven in [31, p. 151].

Lemma 27 Let $H$ be a bipartite graph with colour classes $A$ and $B$, let $x$ and $u$ be distinct vertices in $A$, and let $y$ and $v$ be distinct vertices in $B$. Suppose $H$ has a perfect matching $M$, an $M$-ear $(x, y)$-path $P$, and an $M$-ear $(u, v)$-path $Q$. Then $H$ has a perfect matching $M^{\prime}$, an $M^{\prime}$-ear $(x, y)$-path $P^{\prime}$, and an $M^{\prime}$-ear $(u, v)$-path $Q^{\prime}$ such that

a) $P^{\prime}+Q^{\prime}$ is a subgraph of $P+Q$,

b) $M-E(P+Q)=M^{\prime}-E(P+Q)$, and

c) either $P^{\prime} \cap Q^{\prime}$ is a path which is well-fitted to $M^{\prime}$, or $P^{\prime}$ and $Q^{\prime}$ are disjoint.

Suppose $H_{1}$ and $H_{2}$ are disjoint subgraphs of $H$ which are both well-fitted to a perfect matching $M$ of $H$. An $M$-ear path from $H_{1}$ to $H_{2}$ is an $M$-ear path of $H_{1}+H_{2}$ with origin on $H_{1}$ and terminus on $H_{2}$. 
Corollary 28 Suppose $H$ is a $k$-extendible bipartite graph with a perfect matching $M$. If $H_{1}$ and $H_{2}$ are disjoint subgraphs of $H$ which are both well-fitted to $M$, then there exist $k$ internally disjoint $M$-ear paths from $H_{1}$ to $H_{2}$. If in addition $\nu\left(H_{1}\right) \geq 2 k$ [respectively, $\nu\left(H_{2}\right) \geq 2 k$, then the $k$ paths can be chosen to have distinct origins [respectively, termini].

Proof. Let $D$ be the digraph corresponding to $H$, where $M$ corresponds $V(D)$. Since $H_{1}$ and $H_{2}$ are well-fitted to $M$, they correspond to subdigraphs $D_{1}$ and $D_{2}$, respectively, of $D$. Since $H$ is $k$-extendible, $D$ is strongly $k$-connected by Theorem 3 . Then there are $k$ internally disjoint dipaths $P_{1}, \ldots, P_{k}$ from $V\left(D_{1}\right)$ to $V\left(D_{2}\right)$ by Theorem $1 . P_{1}, \ldots, P_{k}$ correspond to paths of $H$ which are well-fitted to $M$. Removing both ends of each of these paths gives $k$ internally disjoint $M$-ear paths $Q_{1}, \ldots, Q_{k}$ from $H_{1}$ to $H_{2}$.

Suppose $\nu\left(H_{1}\right) \geq 2 k$. Then $V\left(D_{1}\right) \geq k$; and so $P_{1}, \ldots, P_{k}$ can be chosen to have distinct origins by Theorem 1 . Then $Q_{1}, \ldots, Q_{k}$ also have distinct origins. Similarly, if $\nu\left(H_{2}\right) \geq 2 k$, then $Q_{1}, \ldots, Q_{k}$ can be chosen to have distinct termini.

Lemma 29 (F. 23, p. 43) Suppose $M$ is a perfect matching of a brace $H$ with colour class $A$ and $B$, and $K$ is a subgraph of $H$ with at least four vertices which is well-fitted to $M$. If $x \in A \cap V(K), y \in B \cap V(K)$, and $x z$ is an edge incident with $K$, then there exists an $M$-ear path of $K$ which uses $x z$ but not $y$.

Proof. Let $w z$ and $u y$ in $M$ be incident with $z$ and $y$. Since $\nu(K) \geq 4, K-\{u, y\}$ is nonempty. Also, $H-\{u, y\}$ is 1-extendible by Corollary 17. Then $H-\{u, y\}$ has an $M$-ear path $P$ from $w z$ to $K-\{u, y\}$ by Corollary 28. The required path is $x z w+P$.

Lemma 30 Let $H$ be a brace with a perfect matching $M$, and colour classes $A$ and $B$. Suppose $H_{1}$ and $H_{2}$ are disjoint subgraphs of $H$ which are well-fitted to $M$, and $P$ is an $M$-ear path from $H_{1}$ to $H_{2}$ with origin $s$ and last edge wt, where $s \in A$ and $t \in B$. Then the following statements hold.

a) (F. 23) If $H_{1}$ has at least four vertices, then there exist internally disjoint $M$-ear paths $Q$ and $R$ from $H_{1}$ to $H_{2}$ such that $s$ is the origin of $Q$ or $R$, the origins of $Q$ and $R$ are distinct, and wt is the last edge of $Q$ or $R$.

b) If $\mathrm{H}_{2}$ has at least four vertices, then there exist internally disjoint $M$-ear paths $Q$ and $R$ from $H_{1}$ to $H_{2}$ such that $s$ is the origin of $Q$ or $R$, the termini of $Q$ and $R$ are distinct, and $t$ is the terminus of $Q$ or $R$.

Proof. We will only prove (a). The proof of (b) is similar. By Corollary 28 there exist internally disjoint $M$-ear paths $Q^{\prime}$ and $R^{\prime}$ from $H_{1}$ to $H_{2}$ having distinct origins.

Suppose $s$ is not the origin of $Q^{\prime}$ or $R^{\prime}$. If $P$ and $Q^{\prime}+R^{\prime}$ are disjoint, then $P$ and $Q^{\prime}$ are the required paths. Otherwise, let $v$ be the first vertex of $P$ on $Q^{\prime}+R^{\prime}$. Note that $v \in B$. We may assume $v$ is on $Q^{\prime}$. Let $y$ be the terminus of $Q^{\prime}$. Then $P[s, v]+Q^{\prime}[v, y]$ and $R^{\prime}$ are internally disjoint $M$-ear paths from $H_{1}$ to $H_{2}$ such that $s$ is the origin of one of them and their origins are distinct. Thus, we may assume $s$ is the origin of $Q^{\prime}$ or $R^{\prime}$.

Suppose $w t$ is not the last edge of $Q^{\prime}$ or $R^{\prime}$. Let $x$ be the last vertex of $P-t$ on $Q^{\prime}+R^{\prime}$. (Such a vertex in $A$ exists since $s$ is on $Q^{\prime}+R^{\prime}$.) We may assume $x$ is on $Q^{\prime}$. Let $s^{\prime}$ be the origin of $Q^{\prime}$. Then $Q^{\prime}\left[s^{\prime}, x\right]+P[x, t]$ and $R^{\prime}$ are the required paths. 
L. 29
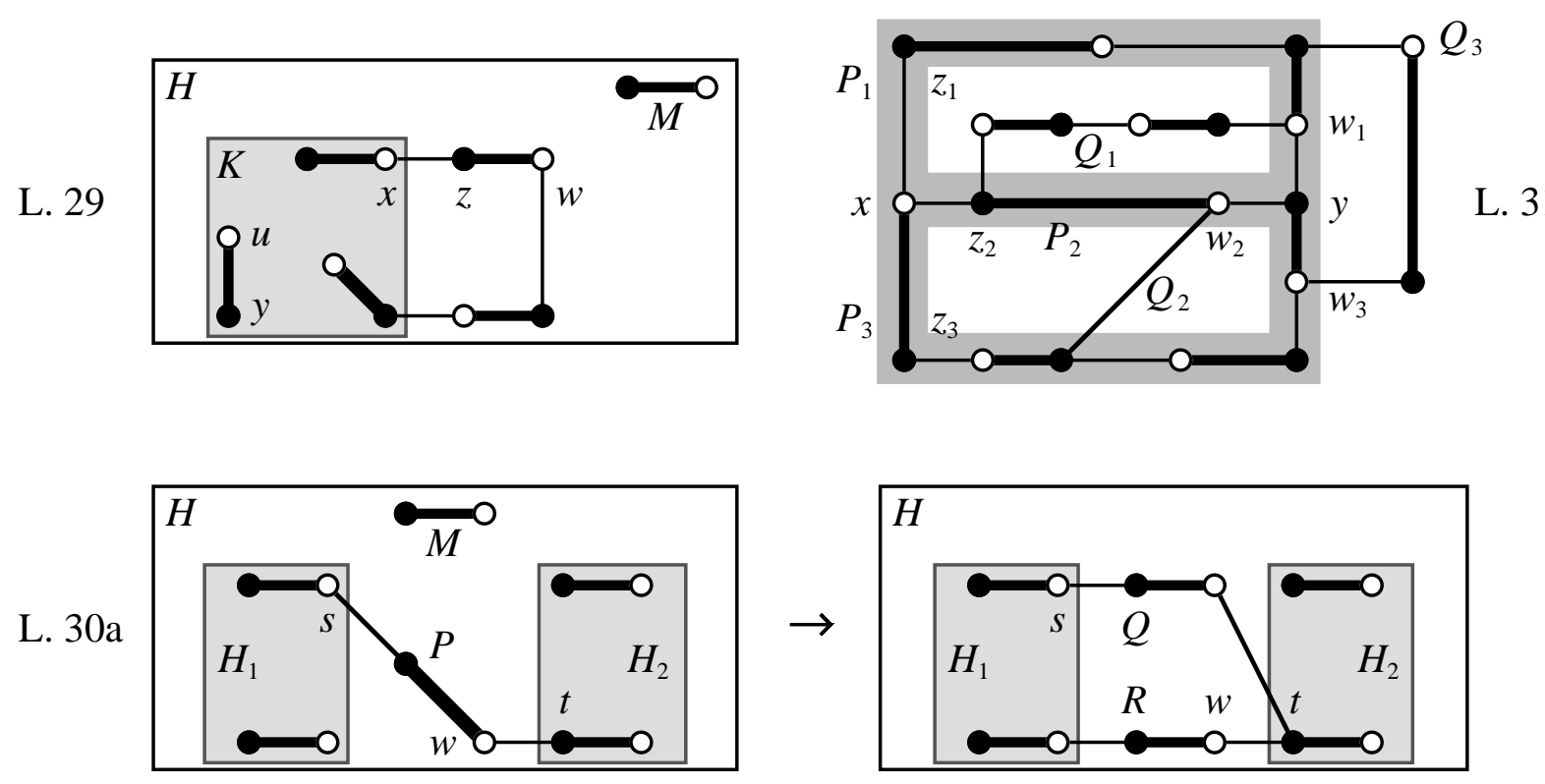

Figure 23: Ear path lemmas.

Let $x$ and $y$ be vertices of a graph $H$. If $P_{1}, \ldots, P_{n}$ are internally disjoint $(x, y)$-paths, then we say $P_{1}+\cdots+P_{n}$ is an $(x, y)^{n}$-fan. Two $(x, y)^{n}$-fans $P_{1}, \ldots, P_{n}$ and $P_{1}^{\prime}, \ldots, P_{n}^{\prime}$ are similar if $P_{i}$ and $P_{i}^{\prime}$ use the same edges incident with $x$ and $y$ for $i=1, \ldots, n$.

Lemma 31 (F. 23) Let $H$ be a brace with colour classes $A$ and $B$. Suppose $x \in A$, $y \in B$, and $x$ and $y$ are nonadjacent. Suppose $n \geq 2$ and $P_{1}, \ldots, P_{n}$ are internally disjoint $(x, y)$-paths such that $P_{1}+\cdots+P_{n}$ is well-fitted to $H$. Let $w_{i} y$ be the last edge of $P_{i}$ for $i=1, \ldots, n$. Then there exist ear paths $Q_{1}, \ldots, Q_{n}$ of $P_{1}+\cdots+P_{n}$, [or a similar well-fitted $(x, y)^{n}$-fan] such that $w_{i}$ is the origin of $Q_{i}$ and the terminus of $Q_{i}$ is not on $P_{i}$ for $i=1, \ldots, n$. (Note that $Q_{1}, \ldots, Q_{n}$ may not be disjoint.)

Proof. Suppose $1 \leq k \leq n$ and there exist ear paths $Q_{1}, \ldots, Q_{k-1}$ of $P_{1}+\cdots+P_{n}$ satisfying the theorem. (If $k=1$, then $\left\{Q_{1}, \ldots, Q_{k-1}\right\}=\emptyset$.) We will prove there exist ear paths $Q_{1}^{\prime}, \ldots, Q_{k-1}^{\prime}, Q_{k}$ of $P_{1}+\cdots+P_{n}$ [or a similar $(x, y)^{n}$-fan] satisfying the theorem. The result will then follow by induction on $k$.

Let $M$ be a perfect matching of $H$ such that $P_{1}+\cdots+P_{n}$ is well-fitted to $M$ and $Q_{1}, \ldots, Q_{k-1}$ are $M$-ear paths. Let $x z_{k}$ be the first edge of $P_{k}$. Lemma 26 can be used to show $P_{1}+\cdots+P_{n}$ is 1-extendible; and so we may assume that its perfect matching $M \cap E\left(P_{1}+\cdots+P_{n}\right)$ contains $x z_{k}$. It then contains $w_{k} y$ as well. In the rest of the proof, all well-fitted subgraphs will be well-fitted to $M$, and all ear paths will be $M$-ear paths.

Consider the well-fitted disjoint subgraphs $H_{2}=\left(\left[P_{1}+\cdots+P_{n}\right]-P_{k}\left[z_{k}, y\right]\right)-y$ and $w_{k} y$, and the ear path $P_{k}\left[w_{k}, z_{k}\right]$ from $w_{k} y$ to $H_{2}$. By Lemma $30 \mathrm{~b}$, there exist internally disjoint ear paths $P$ and $Q_{k}$ from $w_{k} y$ to $H_{2}$ such that $P$ and $Q_{k}$ have distinct termini and $z_{k}$ is the terminus of $P$. 
L. $32 \mathrm{a}$
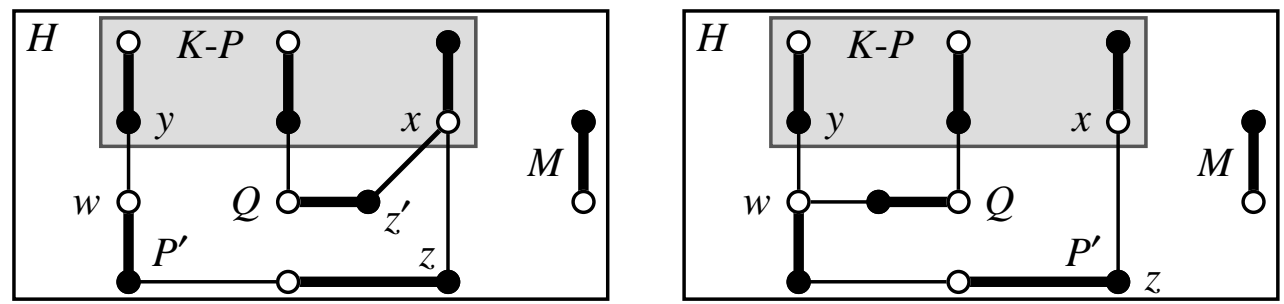

L. $32 b$

L. 33
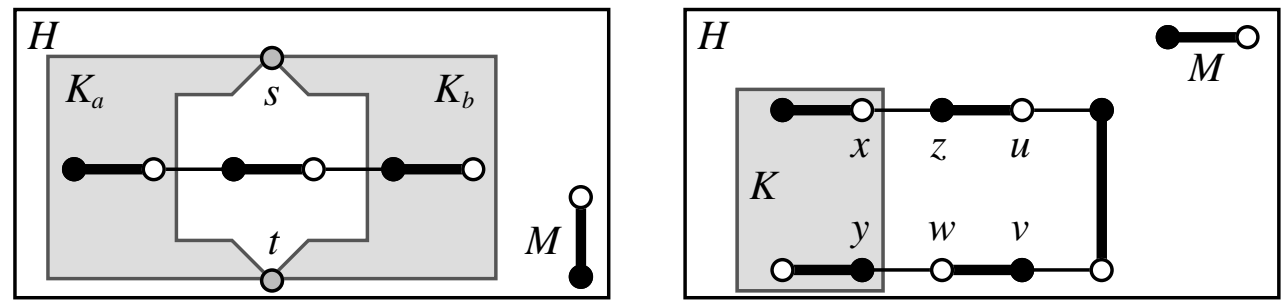

L. 34

Figure 24: More ear path lemmas.

Let $P_{k}^{\prime}=x z_{k}+P+w_{k} y$ and $F=P_{1}+\cdots+P_{k-1}+P_{k}^{\prime}+P_{k+1}+\cdots+P_{n}$. Then $F$ is well-fitted to $H$ and similar to $P_{1}+\cdots+P_{n}$. Furthermore, $Q_{k}$ is an ear path of $F$ with origin $w_{k}$ such that its terminus is not on $P_{k}^{\prime}$.

But now the ear paths $Q_{1}, \ldots, Q_{k-1}$ of $P_{1}+\cdots+P_{n}$ may not be ear paths of $F$; and so they may need modification. Let $z$ be the terminus of $Q_{i}$, where $1 \leq i \leq k-1$. If $z$ is not on $P_{k}$, then let $z^{\prime}$ be the first vertex of $Q_{i}$ after $w_{i}$ which is on $F$, and let $Q_{i}^{\prime}=Q_{i}\left[w_{i}, z^{\prime}\right]$. If $z$ is on $P_{k}$, then let $z^{\prime}$ be the first vertex of $Q_{i}+P_{k}\left[z, z_{k}\right]$ after $w_{i}$ which is on $F$, and let $Q_{i}^{\prime}=\left(Q_{i}+P_{k}\left[z, z_{k}\right]\right)\left[w_{i}, z^{\prime}\right]$. In either case $Q_{i}^{\prime}$ is an ear path of $F$ such that $w_{i}$ is the origin of $Q_{i}^{\prime}$ and the terminus of $Q_{i}^{\prime}$ is not on $P_{i}$.

In a future application of Lemma 31 to a given $(x, y)^{n}$-fan $P_{1}+\cdots+P_{n}$, we will be able to replace $P_{1}+\cdots+P_{n}$ by any similar $(x, y)^{n}$-fan. Hence, we will be able to assume $Q_{1}, \ldots, Q_{n}$ are ear paths of $P_{1}+\cdots+P_{n}$.

Lemma 32 (F. 24) Let $K$ be a well-fitted subgraph of a brace $H$ with colour classes $A$ and $B$. Suppose $K$ can be obtained from an even cycle via a sequence of ear path additions. Let $P$ be a 2-path of $K$ with first edge $x z$ and last edge wy, where $x \in A$ and $y \in B$. Then $K-P$ is well-fitted to $H, \nu(K-P) \geq 4$, and the following statements hold.

a) If $x z^{\prime}$ is an edge which is not on $K$, then there exist internally disjoint ear paths $P^{\prime}$ and $Q$ of $K-P$ such that $x z$ and $x z^{\prime}$ are on $P^{\prime}+Q$ and wy is the last edge of $P^{\prime}$.

b) If $x z \neq w y$, then there exists an ear path $P^{\prime}$ of $K-P$ which uses $x z$ and wy, and an ear path $Q$ of $[K-P]+P^{\prime}$ with origin $w$ and terminus in $V(K)-V\left(P^{\prime}\right)$.

Proof. Lemma 26 can be used show $K-P$ is well-fitted to $H$. Since $P$ is a 2-path, $d(x) \geq 3$. Then $\nu(K-P) \geq 4$.

We first prove (a). If $z^{\prime}$ is on $K-P$, then we let $P^{\prime}=P$ and $Q=x z^{\prime}$. If $P=x z=w y$, then (a) follows from Lemma 29. Therefore, we may assume $z$ and $z^{\prime}$ are not on $K-P$. 
Choose a perfect matching $M$ of $H$ such that $K-P$ is well-fitted to $M$, and $P$ is an $M$-ear path. Let $u z$ and $u^{\prime} z^{\prime}$ be the edges in $M$ incident with $z$ and $z^{\prime}$. We now apply Lemma 30a to the subgraphs $H_{1}=u z+u^{\prime} z^{\prime}$ and $H_{2}=K-P$ which are well-fitted to $M$, and to the $M$-ear path $P[u, y]$. We then get disjoint $M$-ear paths $Q^{\prime}$ and $R$ from $u z+u^{\prime} z^{\prime}$ to $K-P$ such that $R$ has origin $u, Q^{\prime}$ has origin $u^{\prime}$, and $w y$ is on $R$ or $Q^{\prime}$. Then $x z u+R$ and $x z^{\prime} u^{\prime}+Q^{\prime}$ are the required ear paths of $K-P$.

The proof of (b) is similar, except we start with a perfect matching $M$ of $H$ such that both $K$ and $P$ are well-fitted to $M$, and we use Lemma 30b.

In all future applications of Lemma $32 \mathrm{a}$, it will not matter which of the edges $x z$ and $x z^{\prime}$ is the first edge of $P^{\prime}$ and which is the first edge of $Q$, and we will be able to replace $K$ by $[K-P]+P^{\prime}$. Hence, we will be able to assume $P=P^{\prime}$. Under this assumption, $Q$ will then be an ear path of $K$ whose first edge is $x z^{\prime}$ and whose terminus is not an intermediate vertex of $P$. Similarly, in a future application of Lemma 32b, we will be able to assume $P=P^{\prime}$ and $Q$ is an ear path of $K$ with origin $w$ and terminus in $V(K)-V(P)$.

Lemma 33 (F. 24) Let $H$ be a brace with colour classes $A$ and B. Suppose $H$ has a well-fitted subgraph $K$ which is 1-extendible. Suppose $\{s, t\}$ is a 2-vertex cut of $K$ such that $K-\{s, t\}$ has only two components $K_{a}$ and $K_{b}$. If $K_{a}$ has a vertex in $A$ and $K_{b}$ has a vertex in $B$, then there exists an ear path of $K$ with origin on $K_{a}$ and terminus on $K_{b}$.

Proof. We will only prove the lemma when $\{s, t\} \subset A$. The other cases are similar. Choose an edge $s v$ of $K$ such that $v$ is on $K_{a}$. Since $K$ is 1-extendible, there exists a perfect matching $M^{\prime}$ of $K$ containing $s v$. Let ty be the edge in $M^{\prime}$ incident with $t$.

Suppose $y$ is on $K_{a}$. Then $M^{\prime} \cap E\left(K_{b}\right)$ is a perfect matching of $K_{b}$. Hence, $V\left(K_{b}\right)$ contains an equal number of vertices in $A$ and $B$. It follows that no perfect matching of $K$ can include an edge with one end in $K_{b}$ and the other end in $\{s, t\}$. But now we have contradicted the assumption that $K$ is 1-extendible. Therefore, $y$ is in $K_{b}$.

Since $K$ is a well-fitted subgraph, $M^{\prime}$ is contained in some perfect matching $M$ of $H$. Then $K_{a}-v$ and $K_{b}+t y$ are well-fitted to the perfect matching $M-\{s v\}$ of $H-\{s, v\}$, and $V\left(K_{a}-v\right)$ is nonempty because $K_{a}$ has a vertex in $A$. Furthermore, $H-\{s, v\}$ is 1-extendible by Corollary 17. Now Corollary 28 implies $H-\{s, v\}$ has an $(M-\{s v\})$-ear path $P$ from $K_{a}-v$ to $K_{b}+t y$. Since $t$ is in $A$, it is not the terminus of $P$. Hence, $P$ is the required ear path of $K$.

Lemma 34 (F. 24) Let $H$ be a brace with colour classes $A$ and $B$, and a perfect matching $M$. Suppose $K$ is a subgraph of $H$ such that $K$ is well-fitted to $M$, and $H-V(K)$ is 1-extendible. If $x z$ and $w y$ are edges incident with $K$ such that $x \in A \cap V(K)$ and $y \in B \cap V(K)$, then there exists an $M$-ear path of $K$ using $x z$ and wy.

Proof. Let $u z$ and $w v$ be the edges in $M$ incident with $z$ and $w$. If $u z=w v$, then $x z w y$ is the required path. Suppose $u z \neq w v$. Since $H-V(K)$ is 1-extendible, $H-V(K)$ has an $M$-ear path $P$ from $u z$ to $w v$ by Corollary 28. Then $x z u+P+v w y$ is the required path. 


\section{Plane Graphs}

In this section we state some classical results and prove some technical lemmas about plane graphs. They are needed in the proof of the Main Theorem. We assume the reader knows the basics of plane graph theory. (See Tutte [48] or Bondy and Murty [3].)

A planar graph is a graph with a planar embedding, and a plane graph is a graph embedded in the plane. A subgraph of a plane graph is called a plane subgraph.

Theorem 35 Every face boundary of a 2-connected plane graph is a cycle.

Let $r_{1}, r_{2}, s_{1}$, and $s_{2}$ be distinct vertices on a cycle $C$. If we have the cyclic order $r_{1}, s_{1}, r_{2}, s_{2}$ on $C$, then we say $r_{1}$ and $r_{2}$ separate $s_{1}$ and $s_{2}$ on $C$.

Let $K$ be a 2-connected plane subgraph of a plane graph $H$. Suppose $P$ is a path of $H$ having both ends on $K$, but no edges or intermediate vertices on $K$. Then $P$ is contained in the closure of some face of $K$. By Theorem 35 the boundary of the face is a cycle $C$. We say $P$ is across $C$. If the ends of $P$ separate vertices $s_{1}$ and $s_{2}$ on $C$, then we say $P$ separates $s_{1}$ and $s_{2}$ on $C$.

Theorem 36 Let $H$ be a 2-connected plane graph. For every vertex $v$ of $H$, there exists a cyclic permutation $\rho_{v}$ of the edges incident with $v$ such the following properties hold.

a) Distinct edges $e$ and $f$ incident with a vertex $v$ are on a common facial cycle if and only if $\rho_{v}(e)=f$ or $\rho_{v}(f)=e$.

b) If $v_{1} v_{2} \cdots v_{n} v_{1}$ is a facial cycle and $\rho_{v_{1}}\left(v_{n} v_{1}\right)=v_{1} v_{2}$, then $\rho_{v_{2}}\left(v_{1} v_{2}\right)=v_{2} v_{3}$, $\rho_{v_{3}}\left(v_{2} v_{3}\right)=v_{3} v_{4}, \ldots$, and $\rho_{v_{n}}\left(v_{n-1} v_{n}\right)=v_{n} v_{1}$.

If $\rho=\left(e_{1}, e_{2}, \ldots, e_{n}\right)$ and $1 \leq i_{1}<i_{2}<\cdots<i_{k} \leq n$, then $\rho^{\prime}=\left(e_{i_{1}}, e_{i_{2}}, \ldots, e_{i_{k}}\right)$ is called a subpermutation of $\rho$. In the preceding theorem, $\left\{\rho_{v} \mid v \in V(H)\right\}$ is called a planar map for $H$.

Theorem 37 Let $H$ be a 2-connected plane graph, and let $K$ be a 2-connected plane subgraph of $H$. If $\left\{\rho_{v} \mid v \in V(H)\right\}$ is a planar map for $H$, then $K$ has a planar map $\left\{\rho_{v}^{\prime} \mid v \in V(K)\right\}$ such that $\rho_{v}^{\prime}$ is a subpermutation of $\rho_{v}$ for every $v$ in $V(K)$.

A set $\mathcal{C}$ of cycles of a 2 -connected graph $H$ is called a planar mesh if it has the following properties.

1) Every edge of $H$ is on exactly two cycles in $\mathcal{C}$.

2) $\nu(G)-\varepsilon(G)+|\mathcal{C}|=2$.

3) For every vertex $v$ of $H$, there is a cyclic permutation $\rho_{v}$ of the edges incident with $v$ such that for every edge $e$ incident with $v$, some cycle in $\mathcal{C}$ uses both $e$ and $\rho_{v}(e)$.

Theorem 38 Suppose $\mathcal{C}$ is a set of cycles of a 2-connected graph $H$. Then $\mathcal{C}$ is the set of facial cycles for some planar embedding of $H$ if and only if $\mathcal{C}$ is a planar mesh for $H$. A 2-connected graph is planar if and only if it has a planar mesh. 
Let $Z_{2}^{n}$ be the vector space of $n$-tuples over $\{0,1\}$. Let $G$ be a graph with edge set $\left\{e_{1}, \ldots, e_{n}\right\}$. For every cycle $C$ of $G$, let $\bar{a}(C)=\left(a_{1}, \ldots, a_{n}\right)$, where

$$
a_{j}= \begin{cases}0 & \text { if } e_{j} \text { is not on } C, \text { and } \\ 1 & \text { if } e_{j} \text { is on } C,\end{cases}
$$

for $j=1, \ldots, n$. The subspace of $Z_{2}^{n}$ generated by $\{\bar{a}(C) \mid C$ is a cycle of $G\}$ is called the cycle space of $G$.

Theorem 39 If $G$ is a 2-connected plane graph and $\left\{C_{1}, \ldots, C_{m}\right\}$ is its set of interior facial cycles, then $\left\{\bar{a}\left(C_{1}\right), \ldots, \bar{a}\left(C_{m}\right)\right\}$ is a basis for the cycle space of $G$.

Theorem 40 If $C$ is a facial cycle of a 3-connected plane graph $H$, then $H-V(C)$ is connected. If $H$ is also bipartite, then $H-V(C)$ has at least 4 vertices.

Theorem 41 Suppose $R$ and $S$ are disjoint paths and $C$ is a facial cycle of a 2-connected plane graph $K$. Let $r_{1}$ and $r_{2}$ be the ends of $R$, and $s_{1}$ and $s_{2}$ be the ends of $S$. If $r_{1}, r_{2}$, $s_{1}$, and $s_{2}$ are on $C$, then $r_{1}$ and $r_{2}$ do not separate $s_{1}$ and $s_{2}$ on $C$. (Note that $R$ and $S$ may also have intermediate vertices on $C$.)

Lemma 42 A facial cycle of a 1-extendible, bipartite, plane graph $K$ is well-fitted to $K$.

Proof. Let $C=x_{1} y_{1} x_{2} y_{2} \cdots x_{n} y_{n} x_{1}$ be a facial cycle of $K$. We will prove by induction that for every $j$ in $\{1, \ldots, n\}$, there is a perfect matching of $K$ containing $x_{1} y_{1}, \ldots, x_{j} y_{j}$. $C$ is then well-fitted to $K$ because it is well-fitted to the perfect matching for $j=n$.

Since $K$ is 1-extendible, there is a perfect matching containing $x_{1} y_{1}$.

Suppose $2 \leq j \leq n$, and $x_{1} y_{1}, \ldots, x_{j-1} y_{j-1}$ are contained in a perfect matching $M$. Let $x_{j} z$ and $w y_{j}$ be the edges in $M$ incident with $x_{j}$ and $y_{j}$. If $x_{j} z=w y_{j}$, then $M$ is a perfect matching containing $x_{1} y_{1}, \ldots, x_{j-1} y_{j-1}, x_{j} y_{j}$. Hence, we may assume $x_{j} z \neq w y_{j}$. By Corollary 28 there exists an $M$-ear path $P$ with origin $w$ and terminus $z$.

Suppose $x_{1} y_{1} \cdots x_{j-1} y_{j-1}$ and $P$ are not disjoint. Since $x_{1} y_{1} \cdots x_{j-1} y_{j-1}$ is well-fitted to $M$, and $P$ is an $M$-ear path, $w$ is in a different colour class than the first vertex of $P$ on $x_{1} y_{1} \cdots x_{j-1} y_{j-1}$; and so this first vertex is some $y_{i}$ in $\left\{y_{1}, \ldots, y_{j-1}\right\}$. Then $P$ uses the edge $x_{i} y_{i}$ in $M$. Let $R=y_{j} w+P\left[w, y_{i}\right]$ and $S=P\left[x_{i}, z\right]+z x_{j}$. Then $R$ and $S$ are disjoint paths having their ends on $C$ such that the ends $y_{j}$ and $y_{i}$ of $R$ separate the ends $x_{j}$ and $x_{i}$ of $S$ on $C$. But now Theorem 41 is contradicted. Hence, $x_{1} y_{1} \cdots x_{j-1} y_{j-1}$ and $P$ are disjoint. Then shifting $M$ on the alternating cycle $P+z x_{j} y_{j} w$ gives a perfect matching containing $x_{1} y_{1}, \ldots, x_{j-1} y_{j-1}, x_{j} y_{j}$.

Corollary 43 If $C$ is a facial cycle of a plane brace $H$, then $H-V(C)$ is 1-extendible.

Proof. $H$ is 3-connected by Corollary 18. Then $H-V(C)$ is connected and has at least four vertices by Theorem 40. Trivially, $H-V(C)$ has an edge.

Suppose $x y$ is an edge of $H-V(C)$. By Corollary 17, the plane subgraph $H-\{x, y\}$ is 1-extendible. Furthermore, $C$ is a facial cycle of $H-\{x, y\}$. Hence, $C$ is well-fitted to some perfect matching $M$ of $H-\{x, y\}$ by Lemma 42. Then $[M-E(C)] \cup\{x y\}$ is a perfect matching of $H-V(C)$ containing $x y$. 

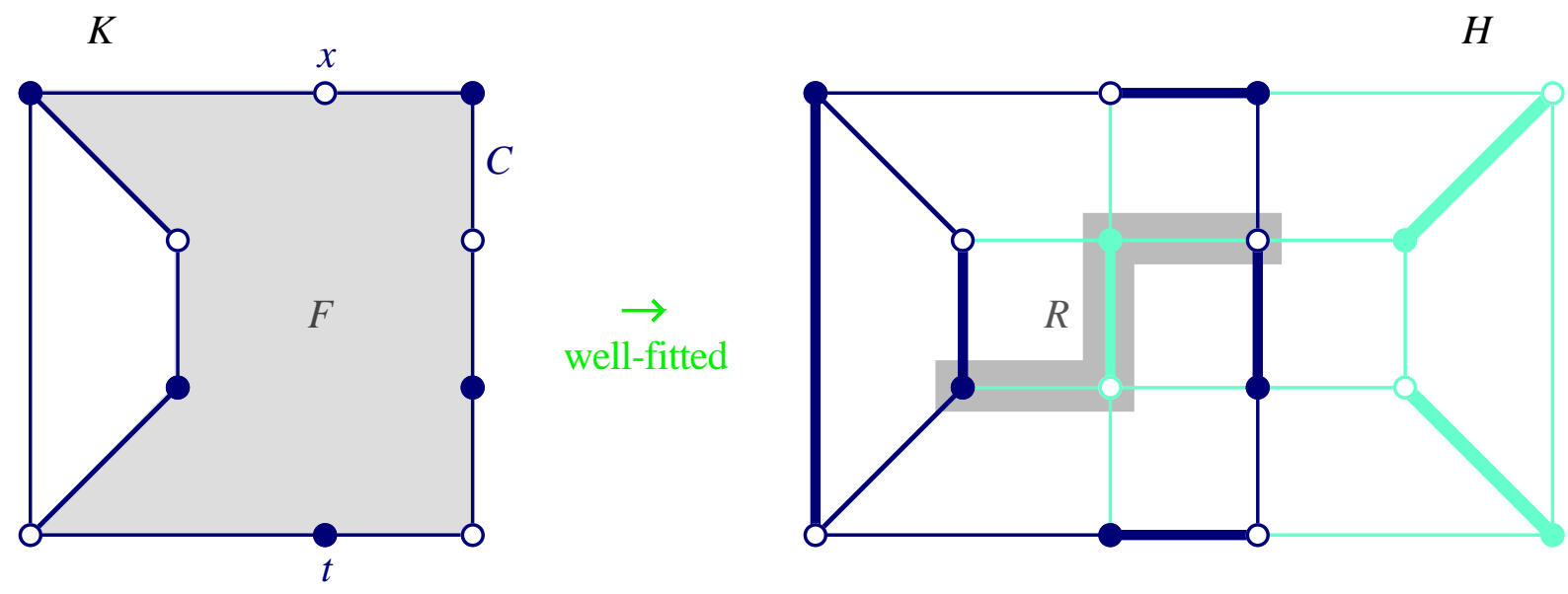

Figure 25: An example for Lemma 44.

Lemma 44 (F. 25) Let $H$ be a plane brace with colour classes $A$ and $B$, and let $K$ be a well-fitted, 2-connected plane subgraph of $H$. Suppose $x$ and $t$ are vertices on a facial cycle $C$ of $K$ such that $x \in A$. If there is a path across $C$ separating $x$ and $t$, then $K$ has an ear path across $C$ separating $x$ and $t$.

Proof. We will only proof this lemma for the case when $t \in B$. The proof is similar when $t \in A$.

Let $F$ be the face of $K$ bounded by $C$. We prove the lemma by induction on the number of faces of $H$ contained in $F$. Let $P$ and $Q$ be the two $(x, t)$-paths of $C$.

Let $u v$ be the first edge of a path across $C$ separating $x$ and $t$. We may assume $u$ is an intermediate vertex of $P$. Since $x \in A$ and $t \in B$, we may assume $u \in A$. By Lemma 29 there is an ear path $R$ of $K$ which uses $u v$ but not $t$. Then $R$ is a path across $C$ because $K$ is a plane subgraph of $H$ and $u v$ is in the closure of $F$. Let $z$ be the terminus of $R$. Note that $z \neq x$ because $z \in B$ and $x \in A$. Furthermore, $z \neq t$ because $R$ does not use $t$. Hence, $z$ is an intermediate vertex of $P$ or $Q$. If $z$ is on $Q$, we are done.

Suppose $z$ is an intermediate vertex of $P$. Then the plane subgraph $K+R$ has a face $F^{\prime}$ contained in $F$ such that $x$ and $t$ are incident with $F^{\prime}$. By the induction hypothesis $K+R$ has an ear path $R^{\prime}$ across $F^{\prime}$ separating $x$ and $t$. If $R^{\prime}$ has an end on $R$, then we can get an ear path across $C$ separating $x$ and $t$ by taking the sum of $R^{\prime}$ and an appropriate subpath of $R$. Otherwise, $R^{\prime}$ is the required path.

Lemma 45 (F. 26a) Let $H$ be a plane brace with colour classes $A$ and B. Suppose $x$ and $t$ are nonadjacent vertices on a facial cycle $C$ of $H$, where $x \in A$. Let $P$ and $Q$ be the two internally disjoint $(x, t)$-paths of $C$. Then $C$ is well-fitted to $H$. Furthermore, if $x z_{r}$ and $x z_{s}$ are edges incident with $C$, then there exist two internally disjoint ear paths of $C$ such that $x z_{r}$ and $x z_{s}$ are the first edges of the ear paths, some intermediate vertex of $P$ is the terminus of one of the ear paths, and some intermediate vertex of $Q$ is the terminus of the other ear path. 


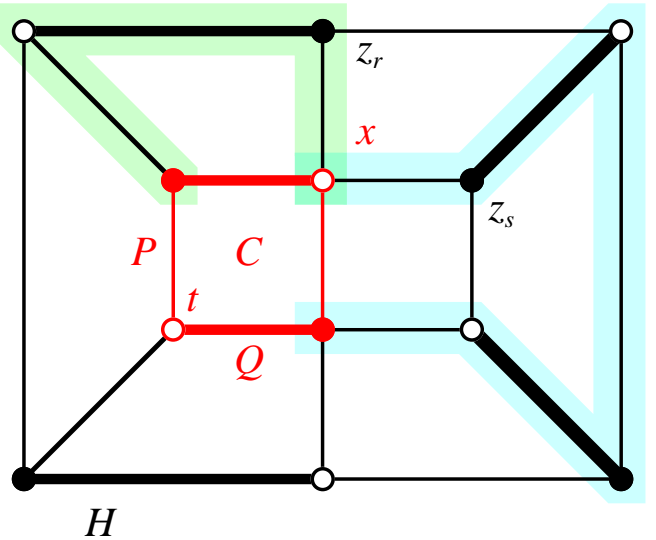

(a)

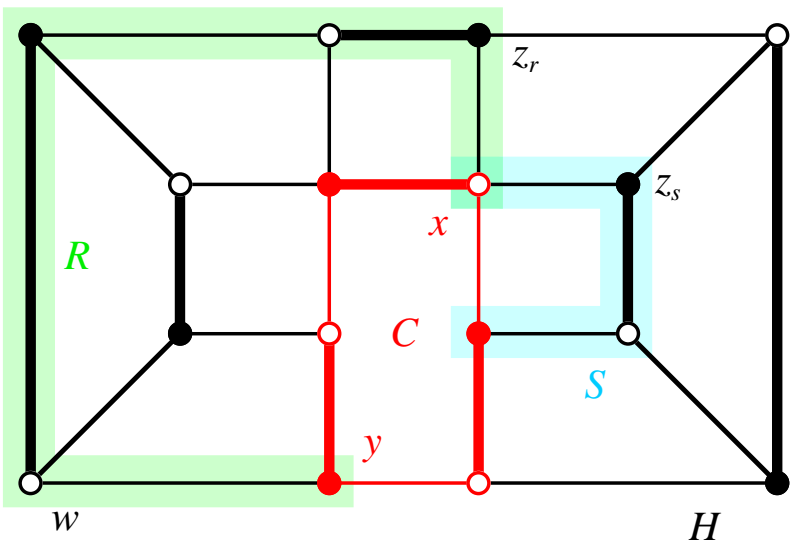

(b)

Figure 26: Examples for Lemmas 45 and 46.

Proof. $C$ is well-fitted to some perfect matching $M$ of $H$ by Lemma 42. Let $w_{r} z_{r}$ and $w_{s} z_{s}$ be the edges in $M$ incident with $z_{r}$ and $z_{s}$. Since $C$ has at least 4 vertices, Corollary 28 implies that there exist disjoint $M$-ear paths $R^{\prime}$ and $S^{\prime}$ from $w_{r} z_{r}+w_{s} z_{s}$ to $C$, where $w_{r}$ is the origin of $R^{\prime}$ and $w_{s}$ is the origin of $S^{\prime}$. Let $R=x z_{r} w_{r}+R^{\prime}$ and $S=x z_{s} w_{s}+S^{\prime}$. Then $R$ and $S$ are internally disjoint ear paths of $C$ having first edges $x z_{r}$ and $x z_{s}$.

Let $y_{r}$ and $y_{s}$ be the termini of $R$ and $S$, respectively. If $\left\{y_{r}, y_{s}\right\}$ consists of an intermediate vertex of $P$ and an intermediate vertex of $Q$, then we are done. If not, then we may assume both $y_{r}$ and $y_{s}$ are on $P$, and we have the order $x, y_{r}, y_{s}, t$ on $P$. Since $x$ and $t$ are nonadjacent and $x \in A$, there exists an intermediate vertex $y$ of $Q$ in $B$. Corollary 18 implies there is an edge $w y$ which is not on $C$. Let $w v$ be the edge in $M$ incident with $w$.

Suppose $w v$ is not on $R$ or $S$. Since $C$ is a facial cycle, $H-V(C)$ is 1-extendible by Corollary 43. Then Corollary 28 implies $H-V(C)$ has an $M$-ear path $S_{1}^{\prime}$ with origin $u$ on $(R+S)-\left\{x, y_{r}, y_{s}\right\}$ and terminus $v$. Let $S_{1}=S_{1}^{\prime}+v w y$. On the other hand, if $w v$ is on $R$ or $S$, we let $S_{1}=w y$ and $u=w$.

If $u$ is on $R$, then the ends $y$ and $y_{r}$ of $S_{1}+R\left[u, y_{r}\right]$ separate the ends $x$ and $y_{s}$ of $S$ on the facial cycle $C$. But this contradicts Theorem 41. Therefore, $u$ is on $S$. Then $S[x, u]+S_{1}$ is an $M$-ear path whose first edge is $x z_{s}$ and whose terminus $y$ is an intermediate vertex of $Q$. Since we have the order $x, y_{r}, y_{s}, t$ on $P$, the terminus $y_{r}$ of $R$ is an intermediate vertex of $P$. Thus, $R$ and $S[x, u]+S_{1}$ are the required ear paths.

The proof of the following lemma is similar to that of Lemma 45.

Lemma 46 (F. 26b) Let $H$ be a plane brace with colour classes $A$ and B. Suppose $x$ and $y$ are nonadjacent vertices on a facial cycle $C$ of $H$ such that $x \in A$ and $y \in B$. If $x z_{r}$, $x z_{s}$, and wy are edges incident with $C$, then there exist two internally disjoint ear paths $R$ and $S$ of $C$ such that $x z_{r}$ and $x z_{s}$ are on $R+S$, the terminus of $S$ is not $y$, and wy is the last edge of $R$. 


\section{Well-Fitted Subgraphs}

In this section we provide more technical lemmas needed in the proof of the Main Theorem.

First we explain some figure conventions used in the rest of the paper. Figure 30 (p. 57) illustrates these conventions. All remaining figures represent bipartite graphs with colour classes $A$ and $B$, where the light vertices are in $A$ and the dark vertices are in $B$. If a path has an end in $A$ and an end in $B$, then we use the convention that the origin is the end in $A$. A line joining two vertices represents an edge, while a line with three gaps joining two vertices represents a path. (If the two ends of such a path are in the same colour class, then the path may have length 0.) We use the notation of Figure 21 (p. 39) for augmentations. In this context, an edge labelled 1 which is incident with a vertex labelled $x$ represents an $x_{1}$-edge. If $x$ is an end of a path and there is an $x_{1}$-edge on the path, then we label the path with a 1 near $x$. Similar conventions hold for $x_{2}$-edges, $y_{1}$-edges, and $y_{2}$-edges. We remind the reader that the graph of Figure $N i$ is referred to as $H_{N i}$.

Lemma 47 Let $H$ be a brace with colour classes $A$ and $B$, and let $L_{8}$ be the ladder with 8 vertices (F. 22, p. 40). Suppose $H$ has a 4-cycle $C=$ abcda such that $a \in A$ and $H-V(C)$ is connected. Suppose az and wb are edges incident with $C$. Then $H$ has a well-fitted $L_{8}$ bisubdivision with $C+a z+w b$ as a subgraph (F. 27a), or $H$ has a well-fitted $K_{3,3}$ bisubdivision with $C$ as a subgraph.

Proof. We first show that $H$ has a well-fitted $K_{3,3}$ bisubdivision with $C$ as a subgraph, or $H-V(C)$ is 1-extendible.

Suppose $u v$ is an arbitrary edge of $H-V(C)$, where $u \in A$. In this paragraph we show that $u v$ is in a perfect matching of $H-V(C)$, or $H$ has a well-fitted $K_{3,3}$ bisubdivision with $C$ as a subgraph. Since $H$ is a brace, $a b$ and $c d$ are in some perfect matching $M$ of $H$. Since $M-\{a b, c d\}$ is a perfect matching of $H-V(C)$, we may assume $u v \notin M$. Then there are distinct edges $x v$ and $u y$ in $M$, for some $x$ and $y$ in $V(H)-\{a, b, c, d, u, v\}$ (F. 27b). By Corollary 28 there exist internally disjoint $M$-ear paths $P$ and $Q$ such that $P$ is an $(x, b)$-path and $Q$ is an $(x, d)$-path, and there exist internally disjoint $M$-ear paths $R$ and $S$ such that $R$ is an $(a, y)$-path and $S$ is an $(c, y)$-path. If $P+Q$ and $R+S$ are disjoint, then $C+P+Q+R+S+x v u y$ is a $K_{3,3}$ bisubdivision which is well-fitted to $M$. Hence, we may assume $P$ intersects $S$. Let $y^{\prime}$ be the first vertex of $P$ on $S$. Note that $y^{\prime} \in B$. Then $C_{1}=P\left[x, y^{\prime}\right]+S\left[y^{\prime}, y\right]+x v u y$ is an $M$-alternating cycle which is disjoint from $C$. Let $M_{1}$ be the perfect matching obtained by shifting $M$ on $C_{1}$. Then $u v$ is in the perfect matching $M_{1}-\{a b, c d\}$ of $H-V(C)$.

By assumption, $H-V(C)$ is connected. Since $H$ is a brace, $H$ is 3 -connected by Corollary 18. Hence, $H-V(C)$ has an edge. Also, either $H-V(C)$ has at least four vertices, or $H$ is a $K_{3,3}$.

By the previous two paragraphs we can conclude that $H-V(C)$ is 1-extendible, or $H$ has a well-fitted $K_{3,3}$ bisubdivision with $C$ as a subgraph. Since we are done if we have the second possibility, we may assume $H-V(C)$ is 1-extendible. 
(a)
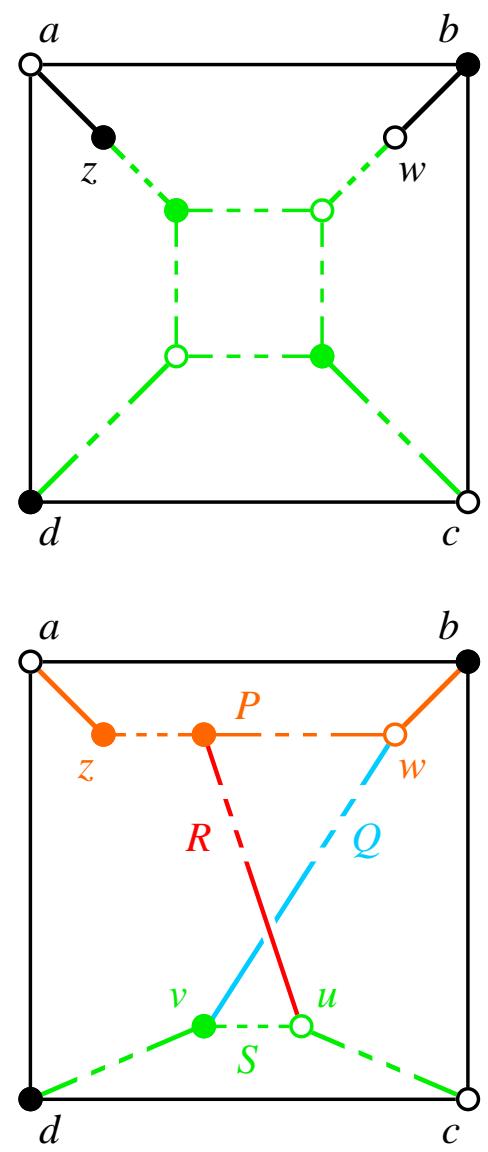

(d) (b)
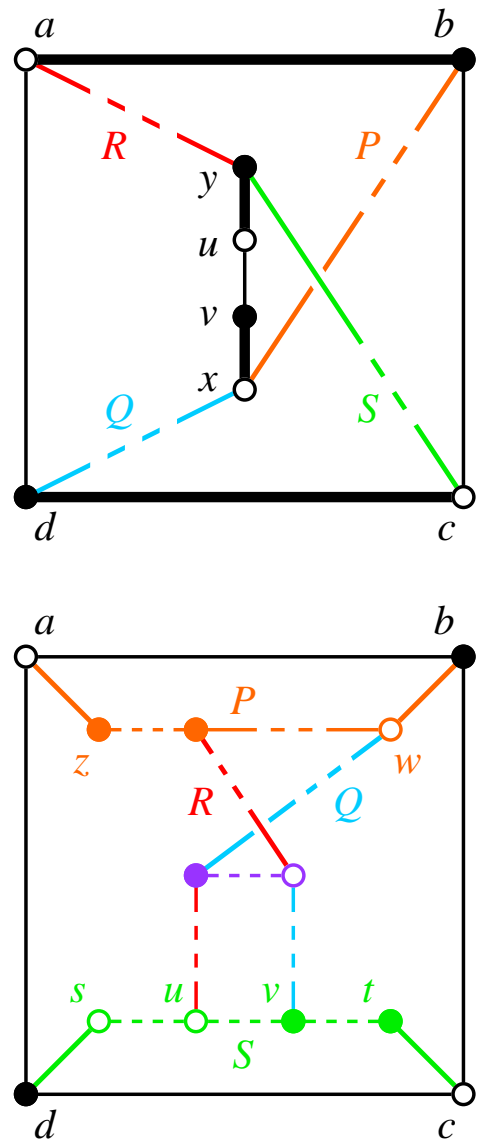

(e) (c)
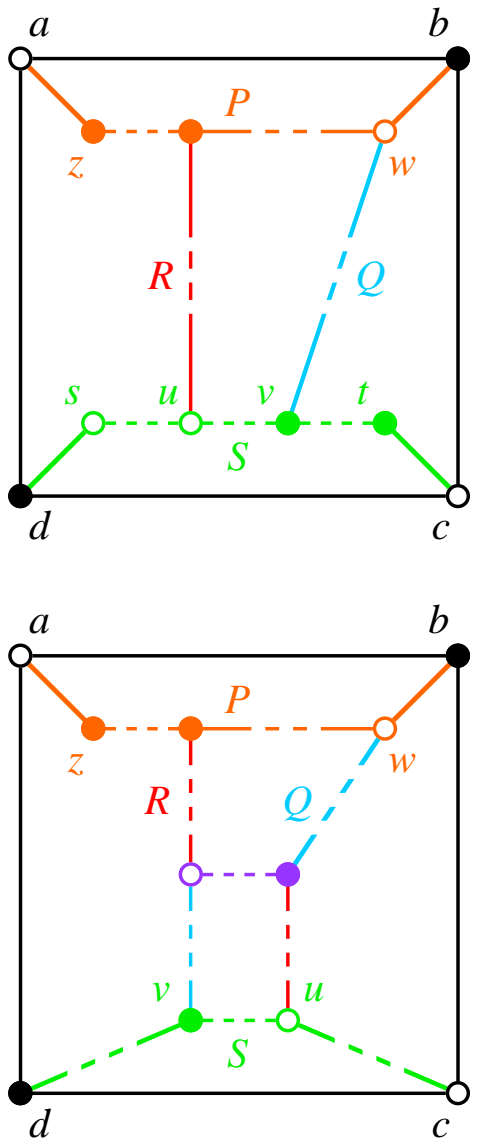

(f)

Figure 27: Lemma 47.

Corollary 18 implies there exist edges $c t$ and $s d$ incident with $C$. Let $M$ be a perfect matching of $H$ such that $C$ is well-fitted to $M$. Since $H-V(C)$ is 1-extendible, Lemma 34 implies that $C$ has an $M$-ear path $P$ using $a z$ and $w b$, and an $M$-ear path $S$ using $c t$ and sd. By Lemma 27 we may assume that either $P$ and $S$ are disjoint, or $P \cap S$ is a path which is well-fitted to $M$. If $P \cap S$ is a path which is well-fitted to $M$, then $C+P+S$ is a well-fitted $K_{3,3}$ bisubdivision with $C$ as a subgraph. Therefore, we may assume $P$ and $S$ are disjoint. We now apply Lemma $32 \mathrm{~b}$ to $C+P+S$ and its 2-path $P$. By the comment after Lemma 32 we may assume $C+P+S$ has an ear path $Q$ with origin $w$ such that its terminus is on $S$. We may also assume $Q$ is an $M$-ear path.

Since $P+a b$ and $S+c d$ are disjoint subgraphs which are well-fitted to $M$ and which each have at least 4 vertices, there exist two disjoint $M$-ear paths from $S+c d$ to $P+a b$ by Corollary 28. Let $R$ be one of these paths which does not have $b$ as its terminus. By Lemma 27 we may assume that either $R \cap Q$ is empty, or $R \cap Q$ is a path which is well-fitted to $M$. Let $u$ be the origin of $R$ and let $v$ be the terminus of $Q$. 
Two possibilities for $R \cap Q$ and two possibilities for the cyclic order of $\{c, d, u, v\}$ on $S+c d$ give us the four possible well-fitted subgraphs $H_{27 c}, H_{27 d}, H_{27 e}$, and $H_{27 f}$. If $H$ has a well-fitted $H_{27 d}$ or $H_{27 e}$, then Lemma 26 can be used to show $H$ has a well-fitted $K_{3,3}$ bisubdivision with $C$ has a subgraph. Similarly, if $H$ has a well-fitted $H_{27 c}$ or $H_{27 f}$, then $H$ has a well-fitted $L_{8}$ bisubdivision with $C+a z+w b$ as a subgraph.

Lemma 48 Suppose $G, G_{1}, \ldots, G_{n}$ are braces, where $n \geq 3$. If $G$ is a 4 -cycle sum of $G_{1}, \ldots, G_{n}$ at the 4-cycle $C=$ abcda, then $G$ has a well-fitted $G_{n}$ expansion.

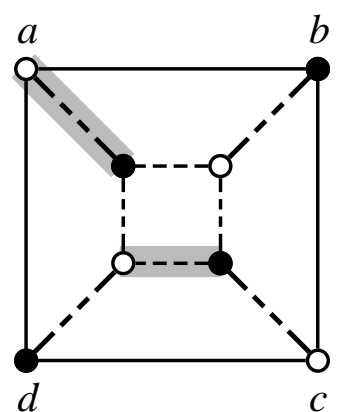

(a)

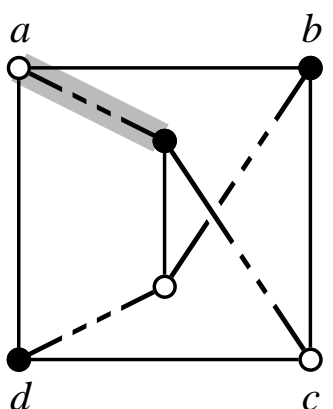

(b)

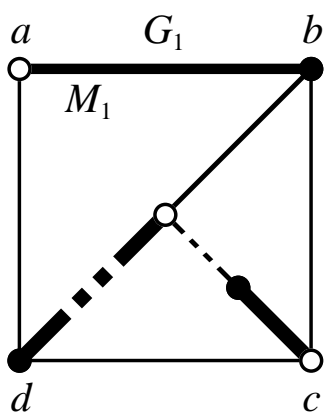

(c)

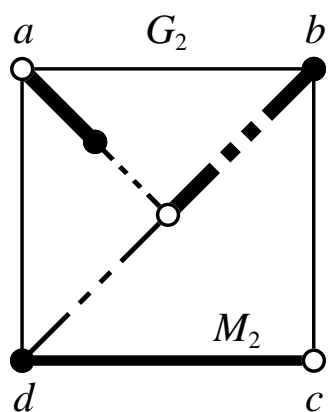

(d)

Figure 28: Lemma 48.

Proof. (This lemma is trivial if $E(C) \subset E(G)$; but we can not make that assumption.) Lemma 47 implies that $G_{1}$ and $G_{2}$ each have a well-fitted $L_{8}$ or $K_{3,3}$ bisubdivision with $C$ as a subgraph. As shown in Figures 28a and 28b, we can apply Lemma 26 to an $L_{8}$ or $K_{3,3}$ bisubdivision to show $G_{1}$ has a well-fitted $H_{28 c}$. It is routine to show that we can choose a perfect matching $M_{1}$ for $G_{1}$ such that $H_{28 c}$ is well-fitted to $M_{1}$, and $a b$ is the only edge in $M_{1}$ on $C$. Similarly, $G_{2}$ has an $H_{28 d}$ and a perfect matching $M_{2}$ such that $H_{28 d}$ is well-fitted to $M_{2}$, and $c d$ is the only edge in $M_{2}$ on $C$. For $3 \leq i \leq n$, there is a perfect matching $M_{i}$ of $G_{i}$ containing $a b$ and $c d$ because $G_{i}$ is a brace. Then $\left(H_{28 c}+H_{28 d}+G_{n}\right)-E(C)$ is a $G_{n}$ expansion which is well-fitted to the perfect matching $\left(\cup_{i=1}^{n} M_{i}\right)-\{a b, c d\}$ of $G$.

Lemma 49 Let $H$ be a brace with colour classes $A$ and $B$. Let $x$ and $y$ be nonadjacent vertices such that $x \in A$ and $y \in B$. Suppose the edges incident with $x$ (respectively, $y$ ) are partitioned into $x_{1}$-edges and $x_{2}$-edges (respectively, $y_{1}$-edges and $y_{2}$-edges). Furthermore, suppose there are at least two $x_{i}$-edges and at least two $y_{i}$-edges for $i=1,2$. If $H$ has a well-fitted $(x, y)^{4}$-fan $K$, then it can be chosen so that it uses two $x_{1}$-edges, two $x_{2}$-edges, two $y_{1}$-edges, and two $y_{2}$-edges.

Proof. Let $n_{x}$ be the absolute value of the difference between the number of $x_{1}$-edges and the number of $x_{2}$-edges on $K$. Define $n_{y}$ similarly. If $n_{x}+n_{y}=0$, then we are done. If $n_{x}+n_{y} \geq 1$ we may assume $K$ uses at most one $x_{1}$-edge. We will prove the lemma by showing $K$ can be replaced by an $(x, y)^{4}$-fan $K^{\prime}$ for which $n_{x}+n_{y}$ is smaller. 
Suppose $K$ does not use an $x_{1}$-edge. Choose an $x_{1}$-edge $e . K$ has an ear path $R$ using $e$ whose terminus $v$ is not $y$ by Lemma 29. Let $S$ be the $(x, y)$-path of $K$ using $v$, and let $K^{\prime}$ be the $(x, y)^{4}$-fan $(K+R)-S[x, v]$. We can use Lemma 26 to show $K^{\prime}$ is well-fitted to $H$. Since $v \neq y, K$ and $K^{\prime}$ use the same edges incident with $y$. Hence, $n_{y}$ is the same for $K$ and $K^{\prime}$. Since $K^{\prime}$ uses one $x_{1}$-edge and three $x_{2}$-edges, $n_{x}+n_{y}$ is smaller for $K^{\prime}$ than for $K$.

Suppose $K$ uses a unique $x_{1}$-edge $f$. Let $P$ be the $(x, y)$-path of $K$ which uses $f$, and let $g$ be an $x_{1}$-edge which is not on $K$. Lemma 26 can be used to show $K$ is 1-extendible. Hence, we can apply Lemma 32 a to $K$, its 2-path $P$, and $g$. By the comment after Lemma 32 we may assume $K$ has an ear path $Q$ whose first edge is $g$ and whose terminus, $z$, is not an intermediate vertex of $P$.

If $z=y$, then $K+Q$ is an $(x, y)^{5}$-fan which uses two $x_{1}$-edges and three $x_{2}$-edges. We may assume $K+Q$ uses more $y_{2}$-edges than $y_{1}$-edges. Hence, there exists an $(x, y)$-path $P_{2}$ of $K$ which uses an $x_{2}$-edge and a $y_{2}$-edge. Let $K^{\prime}=(K+Q)-P_{2}$. Then $K^{\prime}$ is an $(x, y)^{4}$-fan which uses two $x_{1}$-edges and two $x_{2}$-edges. Furthermore, $n_{y}$ for $K^{\prime}$ is the same or one less than $n_{y}$ for $K$. Since $K^{\prime}$ is well-fitted to $H$ by Lemma 26, we are done.

If $z \neq y$, then $z$ is an intermediate vertex of some $(x, y)$-path $P_{1}$ of $K$ other than $P$. Then $(K+Q)-P_{1}[x, z]$ is an $(x, y)^{4}$-fan which uses two $x_{1}$-edges and two $x_{2}$-edges, has the same value of $n_{y}$ as $K$, and is well-fitted to $H$.

Lemma 50 Let $H$ be a brace with colour classes $A$ and $B$. If $x$ and $y$ are nonadjacent vertices such that $x \in A$ and $y \in B$, then $H$ has a well-fitted $(x, y)^{3}$-fan $K$. If the edges of $H$ incident with $x$ are partitioned into $x_{1}$-edges and $x_{2}$-edges, and the edges incident with $y$ are partitioned into $y_{1}$-edges and $y_{2}$-edges, then $K$ can be chosen so that it uses an $x_{1}$-edge, an $x_{2}$-edge, a $y_{1}$-edge, and a $y_{2}$-edge (F. 35a, p. 66). Furthermore, if $K$ uses a unique $x_{j}$-edge e and a unique $y_{k}$-edge $f$, then we may assume $e$ and $f$ are not on the same $(x, y)$-path of $K$.

Proof. Let $M$ be a perfect matching of $H$. There exist vertices $w$ and $z$ such that $x z$ and $w y$ are in $M$. By Corollary 28 there exists an $M$-ear $(w, z)$-path $P$, and there exist internally disjoint $M$-ear $(x, y)$-paths $Q$ and $R$. Let $K=x z+w y+P+Q+R$. Assume $M, P, Q$, and $R$ are chosen so that $E(K)$ is minimal. If $P$ and $Q+R$ are disjoint, then $K$ is an $(x, y)^{3}$-fan which is well-fitted to $M$.

Suppose $P$ and $Q+R$ are not disjoint. Let $v$ be the first vertex of $P$ on $Q+R$. We may assume $v$ is on $Q$. Let $u$ be the last vertex of $P$ on $Q[v, y]$. Since $P$ and $Q$ are $M$-ear paths, it is easy to show $v \in B$ and $u \in A$. Furthermore, $P[w, v]+Q[v, y]+w y$ is an $M$-alternating cycle. Let $M^{\prime}$ be the perfect matching obtained by shifting $M$ on this cycle. Let $w^{\prime}$ be the second to last vertex on $Q$, let $P^{\prime}=Q\left[w^{\prime}, u\right]+P[u$, $z]$, let $Q^{\prime}=Q[x, v]+P[v, w]+w y$, and let $K^{\prime}=x z+w^{\prime} y+P^{\prime}+Q^{\prime}+R$. Observe that $x z$ and $w^{\prime} y$ are in $M^{\prime}, P^{\prime}$ is an $M^{\prime}$-ear $\left(w^{\prime}, z\right)$-path, $Q^{\prime}$ and $R$ are internally disjoint $M^{\prime}$-ear $(x, y)$-paths, and $K^{\prime}$ is a subgraph of $K-Q[v, u]$. But now we have contradicted the minimality of $E(K)$.

The rest of the proof is similar to the proof of Lemma 49, except for a tricky case where Corollaries 17 and 28 are applied to an $(x, y)^{4}$-fan. 
The proof of the following lemma is similar to that of Lemma 50 .

Lemma 51 If $x y, x v$, and $x z$ are edges of a brace $H$, then $H$ has a well-fitted $(x, y)^{3}$-fan $K$ which uses $x y, x v$, and $x z$. If the edges incident with $y$ other than $x y$ are partitioned into $y_{1}$-edges and $y_{2}$-edges, then $K$ can be chosen so that it uses a $y_{1}$-edge and a $y_{2}$-edge.

Lemma 52 Let $x$ and $w$ be vertices in the same colour class of a brace $H$. Suppose the edges incident with $x$ are partitioned into $x_{1}$-edges and $x_{2}$-edges, and suppose there are at least two $x_{i}$-edges for $i=1,2$. Then $H$ has a well-fitted $H_{32 a}$ (p. 62$), H_{32 b}, H_{32 d}$, or $H_{32 e}$ (where the roles of $x_{1}$-edges and $x_{2}$-edges may be interchanged for $H_{32 d}$ and $H_{32 e}$ ).

Proof. Choose a vertex $y$ adjacent to $w$. Then $H$ has a well-fitted $(x, y)^{3}$-fan $K$ by Lemma 50 or 51 . Suppose $w y$ is not on $K$. By Lemma 29 we know $K$ has an ear path $S$ using $w y$. Let $w^{\prime}$ be the origin of $S$, and $P^{\prime}$ be the $(x, y)$-path of $K$ using $w^{\prime}$. Then $\left(K-P^{\prime}\left[w^{\prime}, y\right]\right)+S$ is an $(x, y)^{3}$-fan which uses $w y$, and Lemma 26 can be used to show it is well-fitted to $H$. Therefore, we may assume $w y$ is on $K$.

Let $P$ be the $(x, y)$-path of $K$ using $w y$, and let $w z$ be the second to last edge of $P$. We can use Lemma 26 to show $K$ is 1 -extendible. Thus, we can choose a perfect matching $M$ for $H$ such that $K$ is well-fitted to $M$ and $w z \in M$. Then $K-P$ is well-fitted to $M$. By Lemma 30a there are internally disjoint $M$-ear paths $Q$ and $R$ from $K-P$ to $w z$ such that $x$ is the origin of $Q$ or $R$. Let $K^{\prime}=(K-P[x, z])+Q+R$. Then $K^{\prime}$ is well-fitted to $M$. Observe that $K^{\prime}$ is an $H_{32 a}, H_{32 b}, H_{32 d}$, or $H_{32 e}$ provided that $K^{\prime}$ uses the correct number of $x_{1}$-edges and $x_{2}$-edges. If not, then the completion of the proof is similar to the proof of Lemma 49. (Note that if $K^{\prime}$ is an $H_{32 d}$ or $H_{32 e}$ using only $x_{1}$-edges or only $x_{2}$-edges, then applying the methods in the proof of Lemma 49 to $K^{\prime}$ might result in an $H_{32 a}$ or $H_{32 b}$.)

Lemma 53 Let $G$ be an augmentation of a brace $H$. Suppose $x$ is in $\{a, b, \ldots, \ell\}$, and let $n_{x}$ be the number beside the arrow from Figure 29x to Figure 29x' (pages 55 and 56). If $H$ has a well-fitted $H_{29 x}$ bisubdivision, and $G$ is a type $n_{x}$ augmentation of $H$, then $G$ has a well-fitted $K_{3,3}$ bisubdivision.

Proof. Note that we use the augmentation notation of Figure 21 (p. 39). Let $x$ be in $\{a, b, \ldots, \ell\}$. Suppose $H$ has a well-fitted $H_{29 x}$ bisubdivision $K$, and $G$ is a type $n_{x}$ augmentation of $H$. Then $G$ has an $H_{29 x^{\prime}}$ bisubdivision $K^{\prime}$. It is easy to verify that $K^{\prime}$ (or a subgraph of $K^{\prime}$ if $x \in\{f, j, k\}$ ) is a $K_{3,3}$ bisubdivision.

Choose a perfect matching $M$ of $H$ such that $K$ is well-fitted to $M$. The perfect matching $M \cap E(K)$ of $K$ can be slightly modified to give a perfect matching $M^{\prime}$ of $K^{\prime}$. Then $K^{\prime}$ is well-fitted to the perfect matching $M^{\prime} \cup[M-E(K)]$ of $G$. It follows that $K^{\prime}$ (or a subgraph of $K^{\prime}$ if $x \in\{f, j, k\}$ ) is a $K_{3,3}$ bisubdivision which is well-fitted to $G$.

Lemma 53 will be used throughout the proof of Theorem 56 in the next section. In each instance, we will only refer to the relevant part of Figure 29. 
(a)

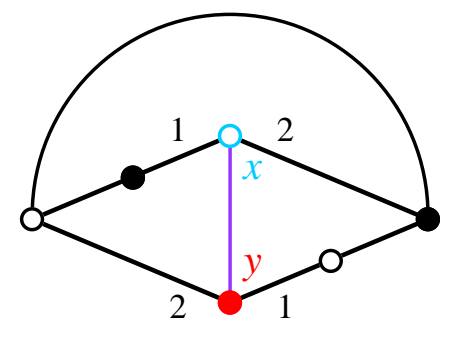

$4 \downarrow$

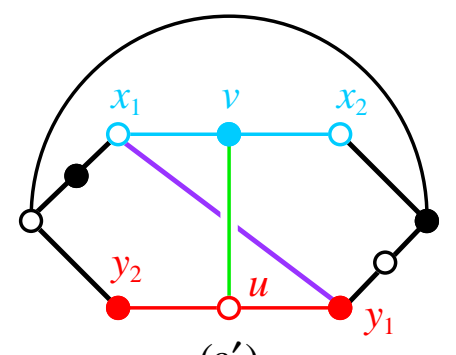

(a')

(d)
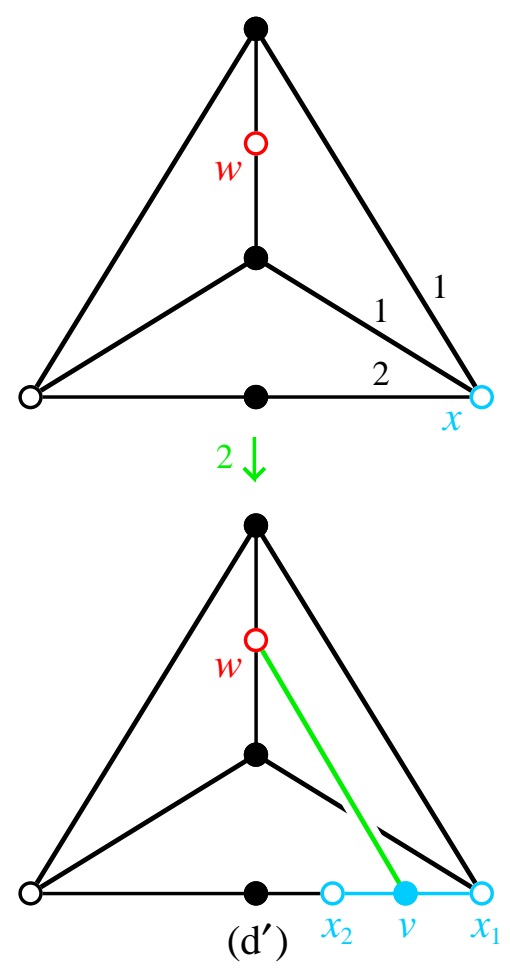

(b)

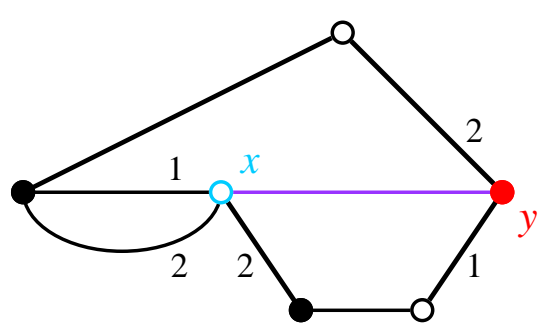

$4 \downarrow$

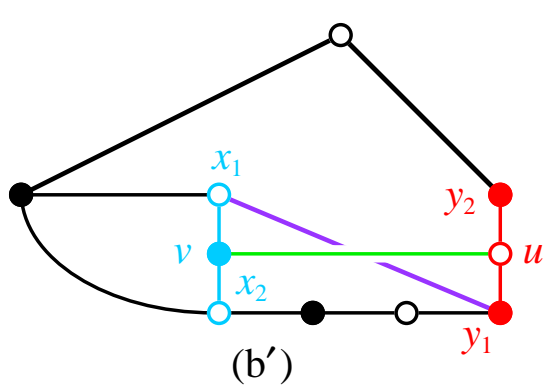

(b')

(e)

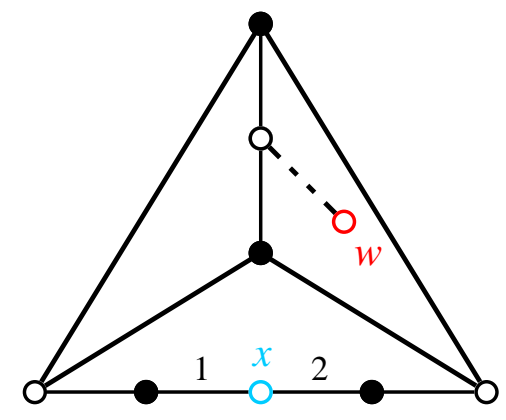

$2 \downarrow$

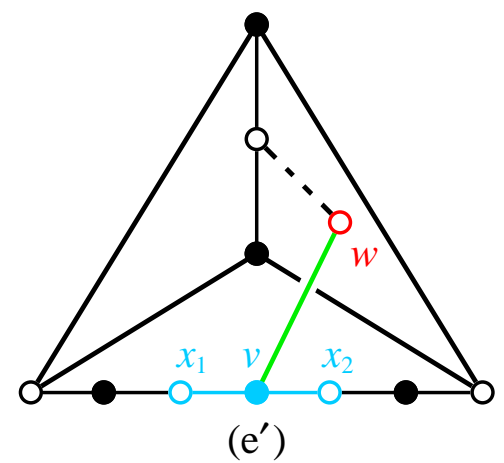

(c)
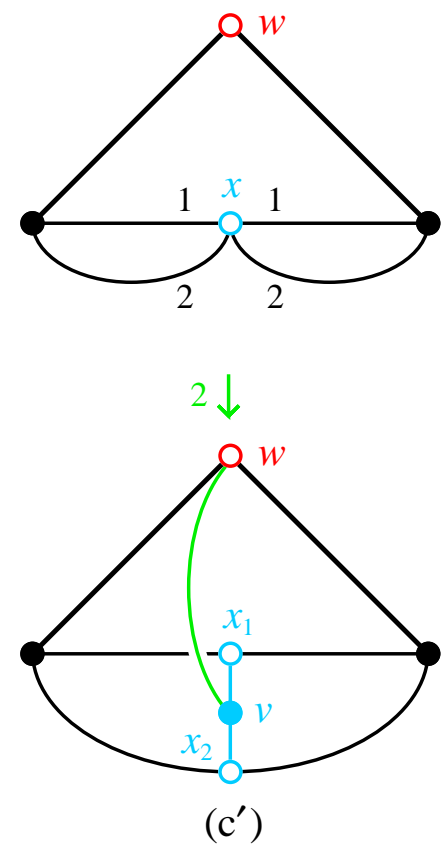

(f)
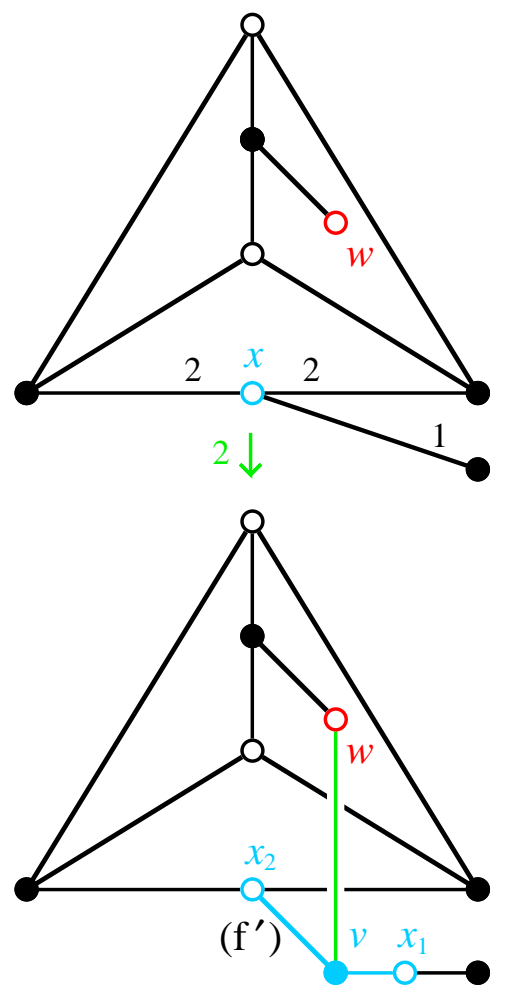

Figure 29: Lemma 53. 
(g)
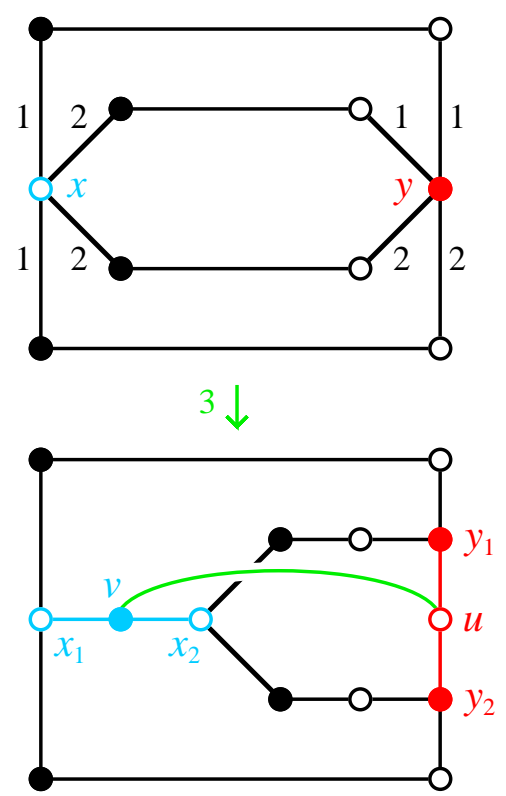

$\left(g^{\prime}\right)$

(j)
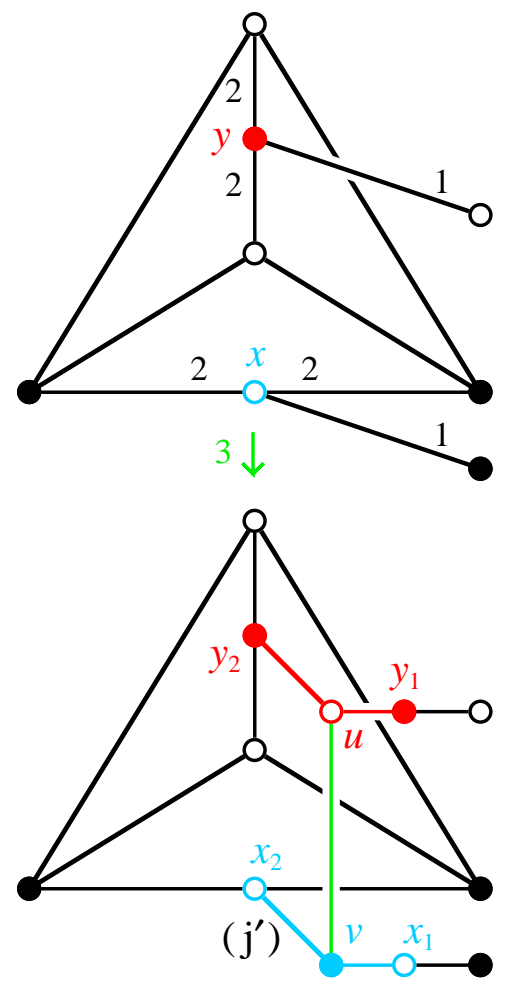

(h)
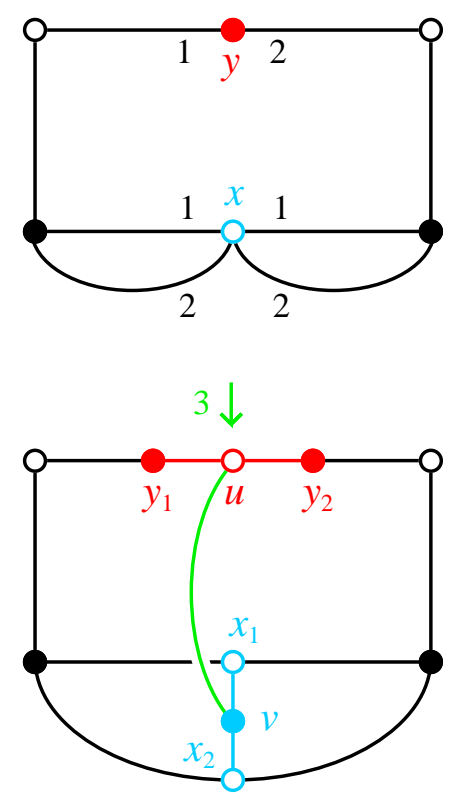

$\left(h^{\prime}\right)$

(k)
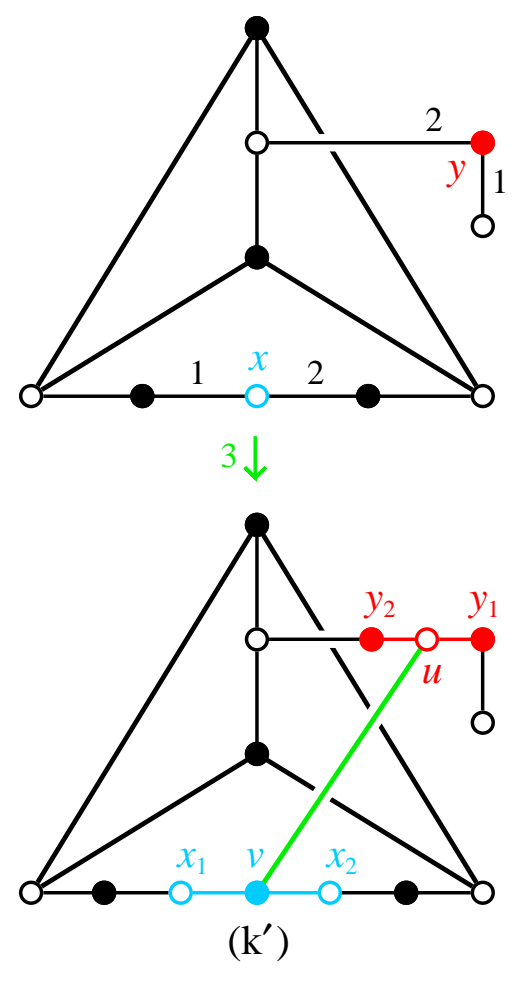

(i)
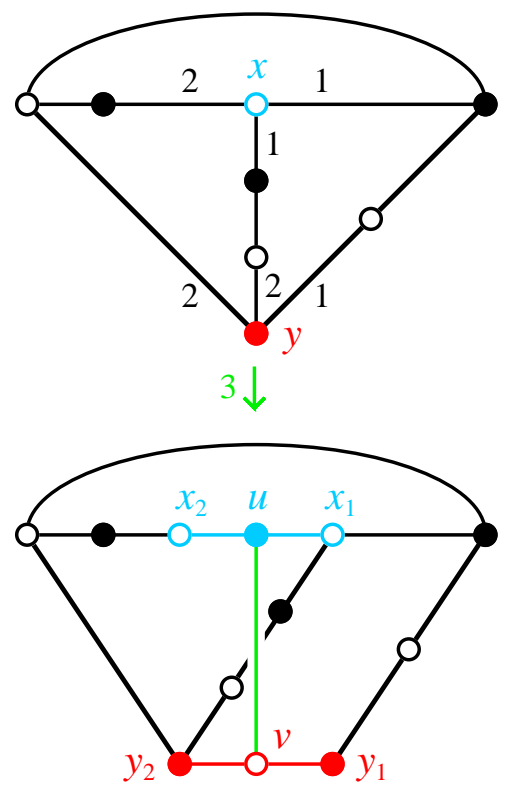

(i')

(
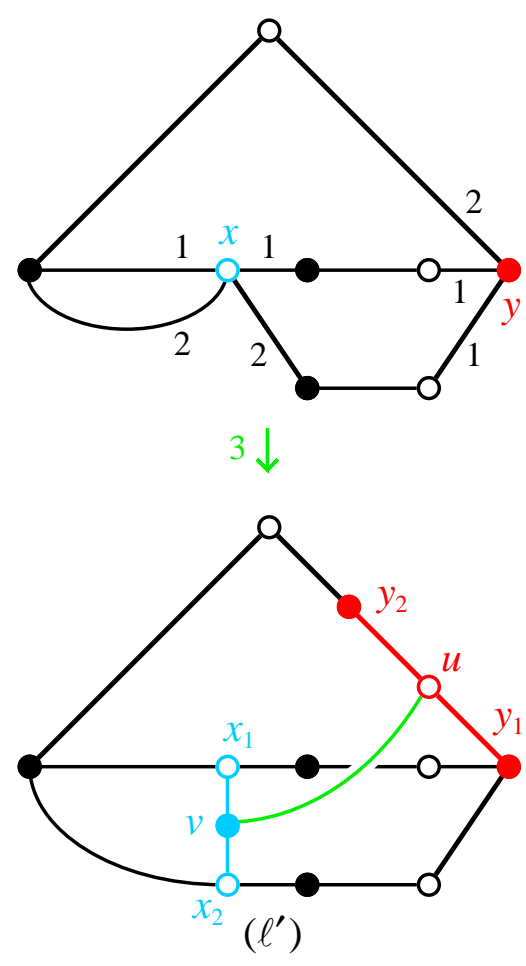

Figure 29: Lemma 53 continued. 


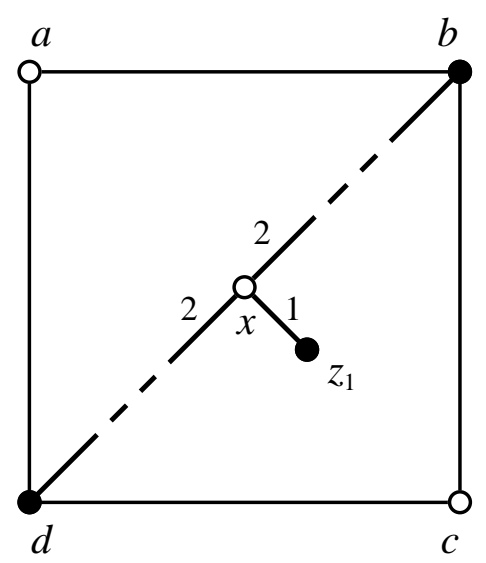

(a)

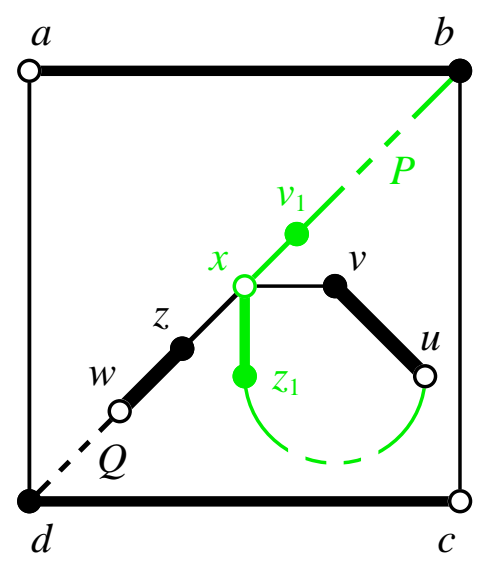

(b)

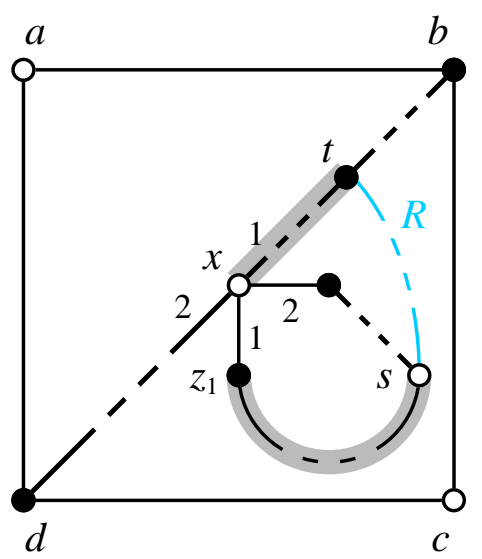

(c)

Figure 30: Lemma 54.

Lemma 54 Let $C=a b c d a$ be a 4-cycle of a brace $H$, and let $x$ be a vertex of $H$ which is

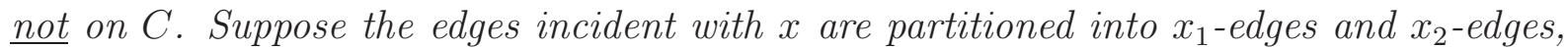
and there are at least two $x_{1}$-edges and at least two $x_{2}$-edges. Then $H$ has a well-fitted $H_{30 a}$ (where the roles of $x_{1}$-edges and $x_{2}$-edges may be interchanged). Furthermore, if $H$ does not have a well-fitted $K_{3,3}$ bisubdivision, then $H_{30 a}$ has an ear path whose origin is in $\{a, c\}$ and whose terminus is not the leaf, $z_{1}$, of $H_{30 a}$ (F. 40a, p. 73).

Proof. We will prove $H$ has a well-fitted $H_{30 a}$ for the case in which $x$ is not adjacent to $b$ or $d$. When $x b$ or $x d$ is an edge the proof is similar and easier.

Since $H$ is a brace, $a b$ and $c d$ are in some perfect matching $M$ of $H$. Let $x z_{1}$ be the edge in $M$ incident with $x$. We may assume $x z_{1}$ is an $x_{1}$-edge. Let $x v$ and $x z$ be $x_{2}$-edges incident with $x$, and let $u v$ and $w z$ be the edges in $M$ incident with $v$ and $z$. By Corollary 28 there exist disjoint $M$-ear paths $P$ and $Q$ from $u v+w z$ to $a b+c d$. We may assume $P$ is a $(u, b)$-path and $Q$ is a $(w, d)$-path. If $x z_{1}$ is not on $P$ or $Q$, then $C+P+Q+u v x z w+x z_{1}$ is an $H_{30 a}$ which is well-fitted to $M$. Hence, we may assume $x z_{1}$ is on $P$ (F. 30b). Let $x v_{1}$ be the other edge on $P$ which is incident with $x$. If $x v_{1}$ is an $x_{2}$-edge, then $C+P\left[z_{1}, b\right]+x z w+Q$ is an $H_{30 a}$ which is well-fitted to $M$. Hence, we may assume $x v_{1}$ is an $x_{1}$-edge (F. 30c). Corollaries 17 and 28 imply that $H-\left\{x, z_{1}\right\}$ has an $M$-ear path $R$ from $P\left(z_{1}, u\right]+u v$ to $C+P\left[v_{1}, b\right]+w z+Q$. Let $s$ and $t$ be the origin and terminus, respectively, of $R$. By symmetry, we may assume $t$ is on $P\left[v_{1}, b\right]$. Then $H_{30 c}-\left(P\left[s, z_{1}\right]+P[x, t]\right)$ is an $H_{30 a}$ which is well-fitted to $M$.

By Corollary 18 we know $a$ and $c$ have degree at least 3 . Hence, $a z_{1}$ and $c z_{1}$ are edges of $H$, or there exists an edge $e$ incident with $a$ or $c$ which is not on $C$ and not incident with $z_{1}$. If $a z_{1}$ and $c z_{1}$ are edges of $H$, then $H_{30 a}+a z_{1}+c z_{1}$ is a $K_{3,3}$ bisubdivision which is well-fitted to $M$. If the edge $e$ exists, then Lemma 29 implies $H_{30 a}$ has an ear path using $e$ such that $z_{1}$ is not the terminus of the ear path. 


\section{The Main Theorem}

In this section we prove the Main Theorem. Most of the work is done in Theorem 56, where we prove that every brace that is not in $\mathcal{G}$ has a well-fitted $K_{3,3}$ bisubdivision.

Theorem 55 Every graph in $\mathcal{G}$ is a brace with an unbalanced $\{-1,1\}$-edge weighting.

Proof. It is routine to verify $H_{14}$ is a brace. (Up to isomorphism, there are only two ways to choose two nonadjacent edges of $H_{14}$.) Lemma 19 and induction are used to show the other graphs in $\mathcal{G}$ are braces.

When showing that every graph in $\mathcal{G}$ has an unbalanced $\{-1,1\}$-edge weighting, it is easier to work with the additive group $\{0,1\}$ than the multiplicative group $\{-1,1\}$. (This will be less confusing if the reader remembers that the " 1 " of $\{-1,1\}$ does not correspond to the "1" of $\{0,1\}$.) Let $w$ be a $\{0,1\}$-edge weighting of a bipartite graph $G$. For a cycle $C$, define $w(C)$ to be the sum $\sum_{e \in E(C)} w(e)$. We say $w$ is unbalanced if $G$ has a perfect matching and $w(C) \equiv 1+\frac{1}{2} \nu(C) \quad(\bmod 2)$ for every alternating cycle $C$. Showing $G$ has an unbalanced $\{-1,1\}$-edge weighting is then equivalent to showing $G$ has an unbalanced $\{0,1\}$-edge weighting.

We use induction to prove that every graph $G$ in $\mathcal{G}$ has an unbalanced $\{0,1\}$-edge weighting. It is routine to verify $H_{14}$ has no 4-cycle, and no alternating cycle of length 8 or 12 . Hence, every alternating cycle of $H_{14}$ has length congruent to 2 modulo 4 . Therefore, we get an unbalanced $\{0,1\}$-edge weighting of $H_{14}$ when every edge is given weight 0 (or every edge is given weight 1 ).

Suppose $G$ is a plane brace. Then the face boundaries of $G$ are cycles by Corollary 18 and Theorem 35. Let $\left\{C_{1}, \ldots, C_{m}\right\}$ be the set of interior facial cycles of $G$ and let $E(G)=\left\{e_{1}, \ldots, e_{n}\right\}$. For every $(i, j)$ in $\{1, \ldots, m\} \times\{1, \ldots, n\}$, let

$$
a_{i j}=\left\{\begin{array}{l}
0 \text { if } C_{i} \text { does not use } e_{j}, \text { and } \\
1 \text { if } C_{i} \text { uses } e_{j} .
\end{array}\right.
$$

Let $r_{i}$ be the least residue modulo 2 of $1+\frac{1}{2} \nu\left(C_{i}\right)$ for $i=1, \ldots, m$. Consider the system

$$
\sum_{j=1}^{n} a_{i j} x_{j}=r_{i}, \quad i=1, \ldots, m,
$$

of linear equations over $Z_{2}$. The set $\left\{\left(a_{i 1}, \ldots, a_{i n}\right) \mid i=1, \ldots, m\right\}$ of $n$-tuples is linearly independent by Theorem 39; and so the rank of the $m \times n$ matrix $\left[a_{i j}\right]$ is $n$. Therefore, the system of linear equations has a solution $\left(x_{1}, \ldots, x_{n}\right)=\left(b_{1}, \ldots, b_{n}\right)$ in $Z_{2}^{n}$. Let $w\left(e_{j}\right)=b_{j}$ for $j=1, \ldots, n$. We will show $w$ is an unbalanced $\{0,1\}$-edge weighting of $G$.

Suppose $M$ is a perfect matching of $G$, and $C$ is an $M$-alternating cycle. We prove $C$ is unbalanced by induction on the number of faces of $G$ in the interior of $C$. If there is only one face in the interior of $C$, then $C$ is its facial cycle; and so $C$ is unbalanced by the choice of $w$. If there are at least two faces in the interior of $C$, then there is an edge $e$ with one or two ends on $C$, and all other points in the interior of $C$. By Lemma 29 there is an $M$-ear path $P$ of $C$ using $e$. All points on $P$ except its ends are in the interior of $C$. 
Let $C_{1}$ and $C_{2}$ be the cycles of $C+P$ using $P$. Then $C_{1}$ and $C_{2}$ are alternating cycles because $C$ is an $M$-alternating cycle and $P$ is an $M$-ear path. Furthermore, the faces of $G$ in the interior of $C$ are partitioned into those in the interior of $C_{1}$ and those in the interior of $C_{2}$. Hence, $C_{1}$ and $C_{2}$ are unbalanced by the induction hypothesis. Therefore,

$$
\begin{aligned}
w(C) & =\sum_{e \in E(C)} w(e) \equiv \sum_{e \in E(C)} w(e)+2 \sum_{e \in E(P)} w(e)=\sum_{e \in E\left(C_{1}\right)} w(e)+\sum_{e \in E\left(C_{2}\right)} w(e) \\
& =w\left(C_{1}\right)+w\left(C_{2}\right) \equiv\left[1+\frac{1}{2} \nu\left(C_{1}\right)\right]+\left[1+\frac{1}{2} \nu\left(C_{2}\right)\right] \equiv \frac{1}{2}\left[\nu\left(C_{1}\right)+\nu\left(C_{2}\right)\right] \\
& =\frac{1}{2}[\nu(C)+2 \nu(P)-2]=\nu(P)-1+\frac{1}{2} \nu(C) \equiv 1+\frac{1}{2} \nu(C) \quad(\bmod 2) .
\end{aligned}
$$

Thus, $C$ is unbalanced.

Suppose $G$ is a 4 -cycle sum of graphs $G_{1}, \ldots, G_{n}$ in $\mathcal{G}$ at $C=a b c d a$, where $n \geq 3$. By the induction hypothesis, $G_{i}$ has an unbalanced $\{0,1\}$-edge weighting $w_{i}$, for $i=1, \ldots, n$. We will use $w_{1}, \ldots, w_{n}$ to construct an unbalanced $\{0,1\}$-edge weighting $w$ of $G$. It suffices to construct $w$ for the case in which $E(C) \subset E(G)$ for the following reason. If a bipartite graph $H$ has an unbalanced $\{0,1\}$-edge weighting $w$, then any subgraph $H^{\prime}$ with a perfect matching has an unbalanced $\{0,1\}$-edge weighting, namely, the restriction of $w$ to $E\left(H^{\prime}\right)$.

Suppose $i$ is in $\{1, \ldots, n\}$. If we switch $w_{i}$ at a vertex $v$, then we get another unbalanced weighting. Hence, we may assume $w_{i}(a b)=w_{i}(b c)=w_{i}(c d)=0$. Since $G_{i}$ is a brace, $G_{i}$ has a perfect matching $M_{i}$ which includes $a b$ and $c d$. Then $C$ is a $w_{i}$-unbalanced alternating cycle of $G_{i}$; and so $w(C) \equiv 1+\frac{1}{2} \nu(C) \equiv 1 \quad(\bmod 2)$ implies $w_{i}(d a)=1$. Then $w=\cup_{i=1}^{n} w_{i}$ is a well-defined $\{0,1\}$-edge weighting of $G$ because $w_{1}, \ldots, w_{n}$ agree on the edges of $C$. Furthermore, $M=\cup_{i=1}^{n} M_{i}$ is a perfect matching of $G$.

We will show that every $M$-alternating cycle $C_{0}$ of $G$ is $w$-unbalanced. If $C_{0}$ is a cycle of some $G_{i}$, then $C_{0}$ is an $M_{i}$-alternating cycle. Then $C_{0}$ is $w_{i}$-unbalanced; and so it is $w$-unbalanced. Suppose $C_{0}$ is not a cycle of $G_{i}$ for $i=1, \ldots, n$. Then we may assume $C_{0}$ uses edges of $G_{1}-E(C)$ and $G_{2}-E(C)$. Since $a b$ and $c d$ are in $M, C_{0}$ must have the form $a b+c d+P_{1}+P_{2}$, where $P_{i}$ is an $M$-ear path using only edges in $G_{i}-E(C)$ for $i=1,2$. We may assume $P_{1}$ is an $(a, d)$-path and $P_{2}$ is a $(c, b)$-path. Let $C_{1}=P_{1}+d a$ and $C_{2}=P_{2}+b c$. For $i=1,2$, let $M_{i}^{\prime}$ be the perfect matching of $G_{i}$ obtained by shifting $M_{i}$ on $C$. Then $C_{i}$ is $w$-unbalanced because it is an $\left(M_{i}^{\prime}\right)$-alternating cycle of $G_{i}$. Hence,

$$
\begin{aligned}
w\left(C_{0}\right) & =w\left(a b+c d+P_{1}+P_{2}\right) \equiv w(a b c d a)+w\left(P_{1}+d a\right)+w\left(P_{2}+b c\right) \\
& =w(C)+w\left(C_{1}\right)+w\left(C_{2}\right) \equiv 1+\left[1+\frac{1}{2} \nu\left(C_{1}\right)\right]+\left[1+\frac{1}{2} \nu\left(C_{2}\right)\right] \\
& \equiv 1+\frac{1}{2}\left[\nu\left(C_{1}\right)+\nu\left(C_{2}\right)\right]=1+\frac{1}{2} \nu\left(C_{0}\right) \quad(\bmod 2) .
\end{aligned}
$$

Since every $M$-alternating cycle is $w$-unbalanced, Theorem 4 implies $w$ is unbalanced. Therefore, all graphs in $\mathcal{G}$ have unbalanced $\{0,1\}$-edge weightings. 
Theorem 56 Every brace has a well-fitted $K_{3,3}$ bisubdivision or is in $\mathcal{G}$.

Proof. Let $G$ be a brace. We prove the theorem by induction on $\varepsilon(G)$. If $G$ is a Möbius ladder (F. 22, p. 40), then it is easy to show $G$ has a well-fitted $K_{3,3}$ bisubdivision. If $G$ is a ladder or a biwheel, then $G$ is in $\mathcal{G}$ because it is planar. Thus, we may assume $G$ is not in $\mathcal{B}$. Then $G$ is an augmentation of some smaller brace $H$ by Theorem 24 .

Suppose $H$ has a well-fitted $K_{3,3}$ bisubdivision. Since $G$ is an augmentation of $H$, there is an expansion $H^{\prime}$ of $H$ and an edge $e$ of $G$ such that $G=H^{\prime}+e$. Then $H^{\prime}$ is well-fitted to $G$ because $V\left(H^{\prime}\right)=V(G)$. (We remind the reader that a bisubdivision of a graph is also an expansion of the graph, and that an expansion of a graph with maximum degree 3 is also a bisubdivision of the graph.) Since $G$ has a well-fitted $H$ expansion, and $H$ has a well-fitted $K_{3,3}$ bisubdivision (expansion), Lemma 23 implies $G$ has a well-fitted $K_{3,3}$ expansion (bisubdivision). Hence, $G$ satisfies the theorem. Therefore, we may assume $H$ has no well-fitted $K_{3,3}$ bisubdivision. Hence, $H$ is in $\mathcal{G}$ by the induction hypothesis. Then either $H=H_{14}$, or $H$ is planar, or $H$ is a 4-cycle sum of three or more smaller braces in $\mathcal{G}$. Thus, there are three possibilities for $H$ and four possibilities for the type of augmentation used in obtaining $G$ from $H$. In the rest of the proof we will deal with these possible cases, as well as one special case. We will use the augmentation terminology of Figure 21 and the figure conventions discussed on pages 1 and 50. In particular, we refer to the graph of Figure $N i$ as $H_{N i}$. We will also make extensive use of Lemma 53. In each instance, we will only refer to the appropriate part of Figure 29 (pages 55 and 56).

Special Case. Suppose $G$ has vertices $a, b, c$, and $d$ such that $a$ and $c$ are in one colour class, $b$ and $d$ are in the other colour class, and $G-\{a, b, c, d\}$ has components $G_{1}^{\prime}, \ldots, G_{n}^{\prime}$, where $n \geq 3$. Let $C$ be the 4-cycle $a b c d a$, and let $G_{i}=C+G\left[V\left(G_{i}^{\prime}\right) \cup\{a, b, c, d\}\right]$, for $i=1, \ldots, n$. Then $G$ is a 4 -cycle sum of $G_{1}, \ldots, G_{n}$ at $C$. Since $G$ is a brace, $G \neq H_{10}$ (p. 36), and $\nu\left(G_{i}\right) \geq 6$ for $i=1, \ldots, n$. Then $G_{1}, \ldots, G_{n}$ are also braces by Lemma 19. By the induction hypothesis, $G_{1}, \ldots, G_{n}$ are all in $\mathcal{G}$, or we may assume $G_{n}$ has a well-fitted $K_{3,3}$ bisubdivision.

Suppose $G_{1}, \ldots, G_{n}$ are in $\mathcal{G}$. Since $H_{14}$ has no 4-cycle, none of these graphs is an $H_{14}$. Hence, each of $G_{1}, \ldots, G_{n}$ is a planar brace, or can be generated from planar braces using 4-cycle sums. Then $G$ is generated from planar braces using 4-cycle sums; and so $G$ is in $\mathcal{G}$.

Suppose $G_{n}$ has a well-fitted $K_{3,3}$ bisubdivision. $G$ has a well-fitted $G_{n}$ expansion by Lemma 48. Then $G$ has a well-fitted $K_{3,3}$ expansion (bisubdivision) by Lemma 23 .

Case 1. Suppose there exists an $H_{14}$ bisubdivision $H_{14}^{\prime}$ which is a well-fitted proper subgraph of $G$. (This case includes the possibility that $H=H_{14}$. This expanded case will be useful in Case 5.) Since $H_{14} \notin \mathcal{B}^{+}$, Theorem 25 implies there exists an augmentation $K$ of $H_{14}$ such that some expansion $K^{\prime}$ of $K$ is well-fitted to $G$ (F. 31a). Since $H_{14}$ only has vertices of degree three, $K$ is a type 1 augmentation of $H_{14}$. Using the symmetry of $H_{14}$ we can show $K$ is an $H_{31 b}$. Furthermore, the figure shows $H_{31 b}$ has a well-fitted $K_{3,3}$ bisubdivision. Then $G$ has a well-fitted $K_{3,3}$ bisubdivision by Lemma 23 . 


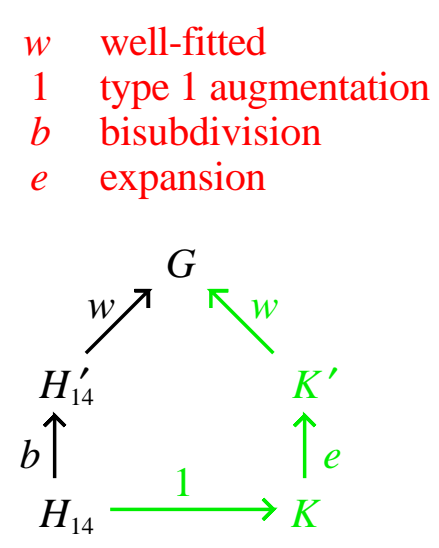

(a)

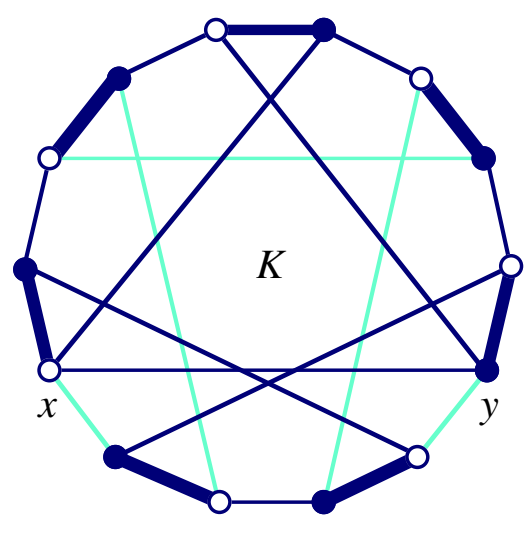

(b)

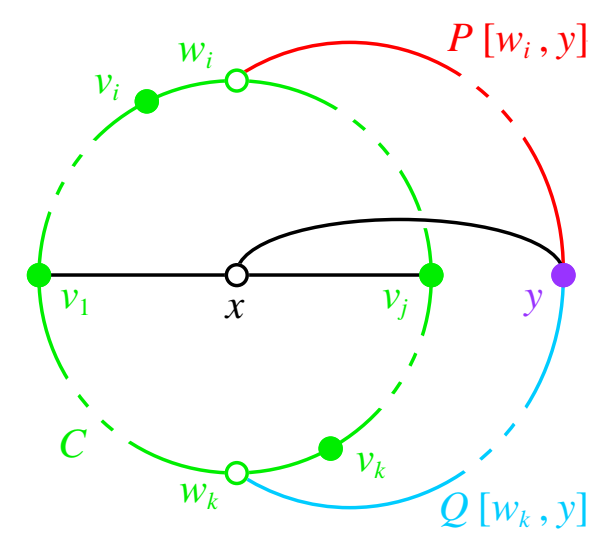

(c)

Figure 31: Cases 1 and 2.

Case 2. Suppose $G$ is a type 1 augmentation of a plane graph $H$. If $x$ and $y$ are on a facial cycle of $H$, then $G=H+x y$ is planar; and hence is in $\mathcal{G}$. Therefore, we may assume $x$ and $y$ are not on a common facial cycle of $H$. Since $H$ is a brace, $H-\{x, y\}$ is 1 -extendible by Corollary 17. Then $H-\{x, y\}$ is 2-connected by Corollary 18; and so every face boundary of $H-\{x, y\}$ is a cycle by Theorem 35. Let $F$ be the face of $H-\{x, y\}$ containing $x$ and let $C=v_{1} w_{1} \cdots v_{n} w_{n} v_{1}$ be the boundary of $F$. Since $x$ and $y$ are not on a common facial cycle of $H$, we know that $y$ is not on $C$, and not in $F$.

$C$ is well-fitted to some perfect matching $M$ of $H-\{x, y\}$ by Lemma 42. We may assume $v_{1} w_{1}, \ldots, v_{n} w_{n}$ are the edges of $C$ in $M$. Then $M \cup\{x y\}$ is a perfect matching of $G$. By Lemma 51, there exist internally disjoint $(x, y)$-paths $P$ and $Q$ such that $P \neq x y \neq Q$ and $P+Q+x y$ is well-fitted to $M \cup\{x y\}$. We may assume $v_{1} w_{1}$ is the first edge of $P$ on $C$. Let $v_{i} w_{i}$ be the last edge of $P$ on $C$, and let $v_{j} w_{j}$ and $v_{k} w_{k}$ be the first and last edges, respectively, of $Q$ on $C$ (F. 31c). We complete this case by showing $C+P\left[w_{i}, y\right]+Q\left[w_{k}, y\right]+x v_{1}+x v_{j}+x y$ is a $K_{3,3}$ bisubdivision which is well-fitted to the perfect matching $M \cup\{x y\}$ of $G$. This subgraph is well-fitted to $M \cup\{x y\}$ because $P\left[w_{i}, y\right]$ and $Q\left[w_{k}, y\right]$ are $M$-ear paths and $C$ is well-fitted to $M$. We will be done if we can show we have the cyclic order $v_{1}, w_{i}, v_{j}, w_{k}$ on $C$.

Consider the disjoint paths $P\left[v_{1}, v_{i}\right]$ and $P\left[w_{i}, y\right]+Q\left[y, v_{j}\right]$ of the plane graph $H-x$. Both these paths have their ends on the facial cycle $C$ of $H-x$. Then by Theorem 41 the ends $v_{1}$ and $v_{i}$ of the first path can not separate the ends $w_{i}$ and $v_{j}$ of the second path. Hence, we have the cyclic order $v_{1}, v_{i}, w_{i}, v_{j}$ on $C$. Similarly, we must have the cyclic order $v_{j}, v_{k}, w_{k}, v_{1}$ on $C$. Therefore, we have the cyclic order $v_{1}, w_{i}, v_{j}, w_{k}$ on $C$.

Case 3. Suppose $G$ is a type 2 augmentation of a plane graph $H$, where $x$ and $w$ are not on a common facial cycle of $H$. By Lemma 52 we know that $H$ has a well-fitted $H_{32 a}$, $H_{32 b}, H_{32 d}$, or $H_{32 e}$. (Note that we have added names for three vertices of $H_{32 e}$.)

Since $H_{32 a}$ is an $H_{29 c}$ bisubdivision, and $H_{32 d}$ is an $H_{29 d}$ bisubdivision, $G$ has a wellfitted $K_{3,3}$ bisubdivision for these possibilities by Lemma 53 . 
Suppose $H$ has a well-fitted $H_{32 b}$. Theorem 38 can be used to show $H_{32 b}$ has the four facial cycles $C_{1}, C_{2}, D_{1}$, and $D_{2}$ shown in Figure 32b. Lemma 44 implies there are ear paths $R_{1}$ and $R_{2}$ across $C_{1}$ and $C_{2}$, respectively, which separate $x$ and $w(\mathrm{~F} .32 \mathrm{c})$. We would like the origin of one of these ear paths to be on $D_{1}$, and the origin of the other to be on $D_{2}$ as shown in Figure 32c. If this is not true, then we may assume the origins of $R_{1}$ and $R_{2}$ are on $D_{2}$. By Lemma 33 we know $H_{32 b}$ has an ear path $R$ with origin on $D_{1}-x$ and terminus on $D_{2}-x$. Furthermore, $R$ is an ear path across either $C_{1}$ or $C_{2}$ which separates $x$ and $w$. Then $R_{1}$ or $R_{2}$ can be replaced by $R$. Hence, we can assume $H$ has a well-fitted $H_{32 c}$. Let $t_{i}$ be the terminus of $R_{i}$ for $i=1$, 2. Since $H_{32 c}-\left(P_{x t_{1}}+P_{x t_{2}}\right)$ is well-fitted to $H$ by Lemma 26, and it is an $H_{29 e}$ bisubdivision, we are done.

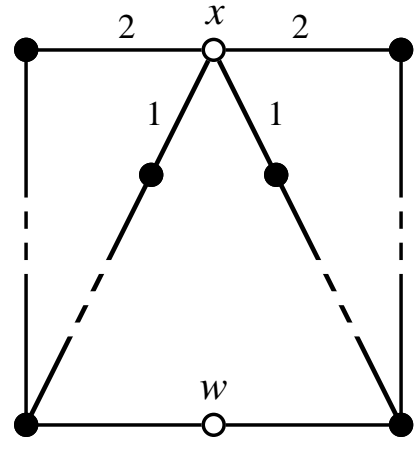

(a)

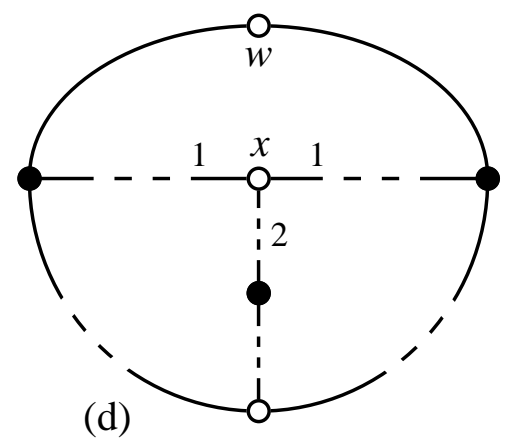

(d)

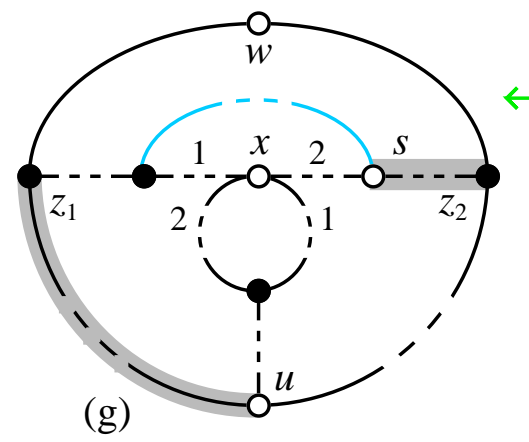

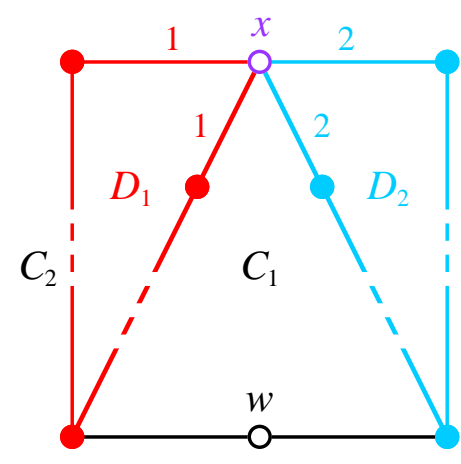

(b)

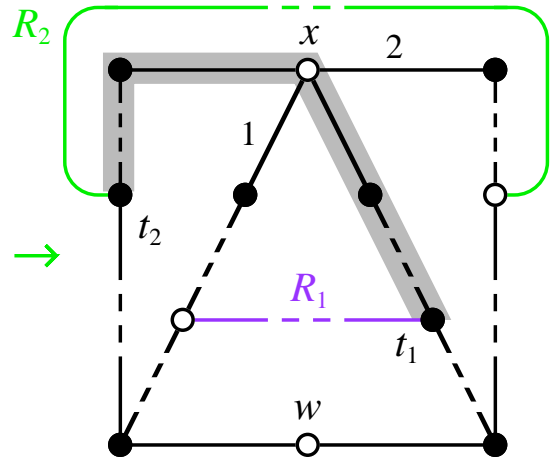

(c)
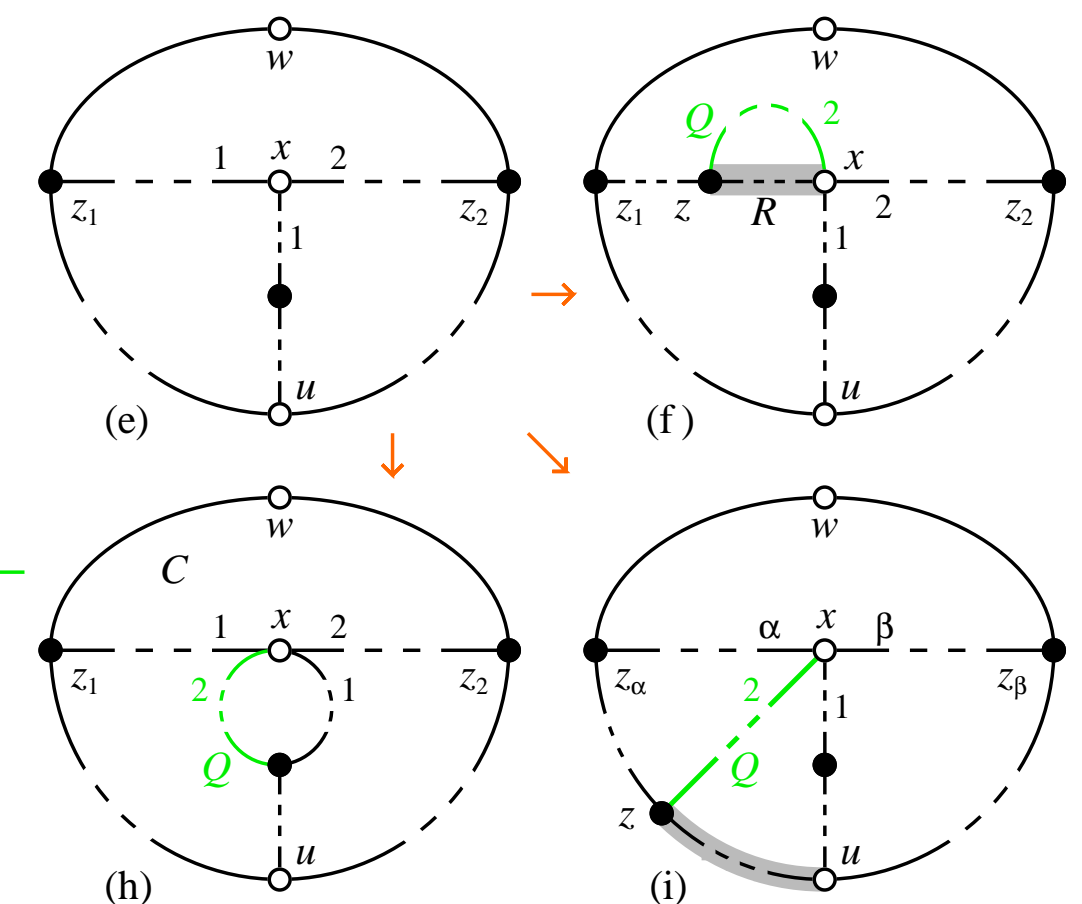

Figure 32: Case 3 
Suppose $H$ has a well-fitted $H_{32 e}$. Since $G$ is a type 2 augmentation of $H$, there are at least two $x_{2}$-edges. Let $e$ be an $x_{2}$-edge which is not on $H_{32 e}$. Lemma 26 implies $H_{32 e}$ is 1-extendible. Hence, we can apply Lemma 32a to $H_{32 e}$, its 2-path $P_{x z_{2}}$, and $e$. By the comment after Lemma 32 we may then assume $H_{32 e}$ has an ear path $Q$ whose first edge is $e$ and whose terminus, $z$, is not an intermediate vertex of $P_{x z_{2}}$. Considering all the possibilities for $z$, we see that $H$ has a well-fitted $H_{32 f}, H_{32 h}$, or $H_{32 i}$ (where $\{\alpha, \beta\}=\{1,2\})$.

Suppose $H$ has a well-fitted $H_{32 f}$. Let $R$ be the 2-path of $H_{32 f}$ with ends $x$ and $z$ which uses an $x_{1}$-edge. Since $H_{32 f}-R$ is well-fitted to $H$ by Lemma 26, and it is an $H_{32 d}$ (with the symmetric roles of $x_{1}$-edges and $x_{2}$-edges reversed), we have a previous subcase.

Suppose $H$ has a well-fitted $H_{32 h}$. Let $C=P_{x z_{1}}+z_{1} w z_{2}+P_{x z_{2}}$. It is easy to show $C$ is a facial cycle of $H_{32 h}$ using Theorem 38. Since $x$ and $w$ are not on a common facial cycle of $H$, Lemma 44 implies there is an ear path across $C$ which separates $x$ and $w$. By symmetry, we may assume the origin, $s$, of this ear path is an intermediate vertex of $P_{x z_{2}}$; and so $H$ has a well-fitted $H_{32 g}$. Since $H_{32 g}-\left(P_{s z_{2}}+P_{u z_{1}}\right)$ is well-fitted to $H$ by Lemma 26, and it is an $H_{29 c}$ bisubdivision, we are done.

Suppose $H$ has a well-fitted $H_{32 i}$. Then Lemma 26 implies $H_{32 i}-P_{u z}$ is a well-fitted $H_{32 a}$ or $H_{32 b}$ subgraph of $H$; and so we have a previous subcase.

Case 4. Suppose $G$ is a type 2 augmentation of a plane graph $H$, where $x$ and $w$ are on a facial cycle $C$ of $H$. By Theorem 36 there is a planar map $\left\{\rho_{v} \mid v \in V(H)\right\}$ for $H$. Let $\rho_{x}=\left(x z_{1}, \ldots, x z_{m}\right)$. Let $P$ and $Q$ be the two internally disjoint $(x, w)$-paths of $C$. We may assume $x z_{1}$ is on $P$ and $x z_{2}$ is on $Q$. Without loss of generality, $x z_{1}$ is an $x_{1}$-edge.

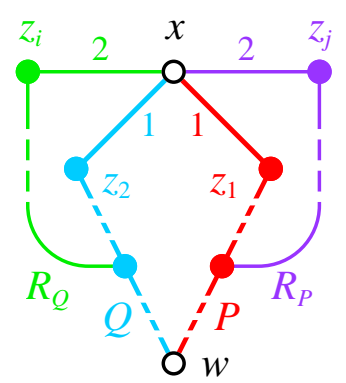

(a)

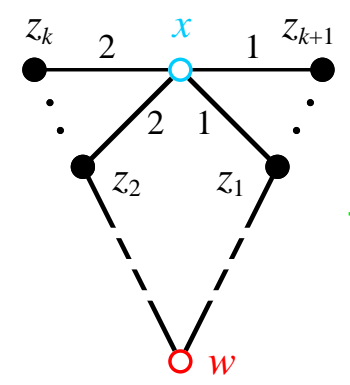

(b)

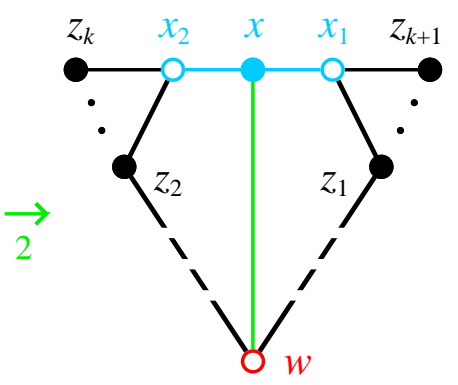

(c)

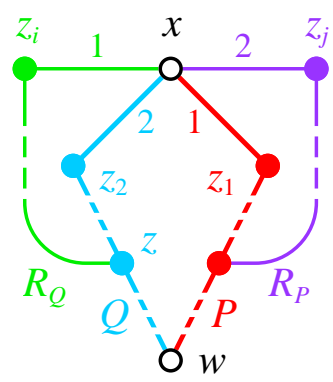

(d)

Figure 33: Case 4

Suppose $x z_{2}$ is an $x_{1}$-edge. By the definition of a type 2 augmentation, there exist $x_{2}$-edges $x z_{i}$ and $x z_{j}$, where $2 \leq i<j \leq m$. Then $H$ has a well-fitted $H_{33 a}$ by Lemma 45 . Since $H_{33 a}$ is an $H_{29 c}$ bisubdivision, we are done. Thus, we may assume $x z_{2}$ is an $x_{2}$-edge.

Suppose there exists $k$ in $\{3, \ldots, m-1\}$ such that $x z_{2}, \ldots, x z_{k}$ are $x_{2}$-edges and $x z_{k+1}, \ldots, x z_{m}, x z_{1}$ are $x_{1}$-edges (F. 33b). Then $G$ is planar as shown in Figure 33c. (This can be rigorously proven by using Theorem 38 and showing that a planar mesh for $H$ can be modified to give a planar mesh for $G$.) Hence, $G \in \mathcal{G}$. 
If such a $k$ does not exist, then there exists an $x_{1}$-edge $x z_{i}$ and an $x_{2}$-edge $x z_{j}$ such that $3 \leq i<j \leq m$ (F. 33d). By Lemma 45, there exist internally disjoint ear paths $R_{P}$ and $R_{Q}$ of $C$ such that $x z_{i}$ and $x z_{j}$ are their first edges, the terminus of $R_{P}$ is an intermediate vertex of $P$, and the terminus of $R_{Q}$ is an intermediate vertex, $z$, of $Q$. We need to show $x z_{i}$ is the first edge of $R_{Q}$, and $x z_{j}$ is the first edge of $R_{P}$, as shown in Figure 33d. Let $K=C+R_{P}+R_{Q}$. By Theorem 37 there is a planar map $\left\{\rho_{v}^{\prime} \mid v \in V(K)\right\}$ for $K$ such that $\rho_{v}^{\prime}$ is a subpermutation of $\rho_{v}$ for every $v$ in $V(K)$. Hence, $\rho_{x}^{\prime}=\left(x z_{1}, x z_{2}, x z_{i}, x z_{j}\right)$. It is easy to verify $R_{Q}+Q[x, z]$ is a facial cycle using Theorem 38. Hence, the first edge of $Q[x, z]$ (namely, $x z_{2}$ ) gets mapped to the first edge of $R_{Q}$ by $\rho_{x}^{\prime}$ or its inverse; and so $x z_{i}$ is the first edge of $R_{Q}$. Therefore, $H$ has a well-fitted $H_{33 d}$. Since $H_{33 d}$ is an $H_{29 c}$ bisubdivision, we are done.
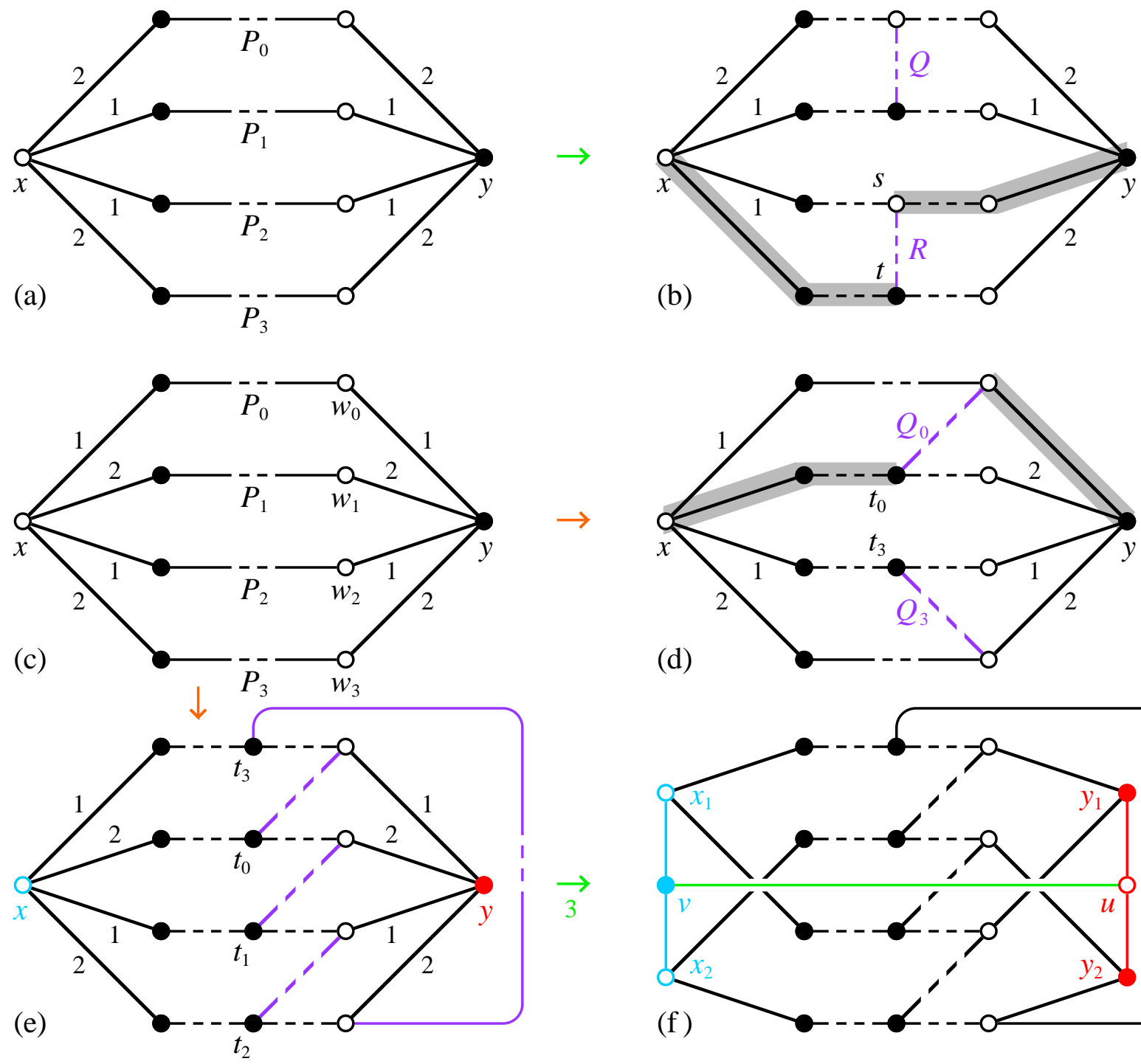

Figure 34: Case 5 
Case 5. Suppose $G$ is a type 3 augmentation of a plane graph $H$, where $x$ and $y$ are not on a common facial cycle of $H$. We first deal with a special subcase. Suppose there exist four internally disjoint $(x, y)$-paths $P_{0}, P_{1}, P_{2}$, and $P_{3}$ such that $P_{0}+P_{1}+P_{2}+P_{3}$ is well-fitted to $H$. We will show that for this special subcase, either $G$ is an $H_{14}$ or we have Case 1.

By Lemma 49 we may assume $P_{0}+P_{1}+P_{2}+P_{3}$ uses two $x_{1}$-edges, two $x_{2}$-edges, two $y_{1}$-edges, and two $y_{2}$-edges. If for every $(i, j)$ in $\{1,2\} \times\{1,2\}$ there is a $k$ in $\{0,1,2,3\}$ such that $P_{k}$ uses an $x_{i}$-edge and a $y_{j}$-edge, then $P_{0}+P_{1}+P_{2}+P_{3}$ is an $H_{29 g}$ bisubdivision, and we are done. Therefore, we may assume (by the symmetry of type 3 augmentations) that two paths in $\left\{P_{0}, P_{1}, P_{2}, P_{3}\right\}$ use both an $x_{1}$-edge and a $y_{1}$-edge. Then the other two paths must use an $x_{2}$-edge and a $y_{2}$-edge. Theorem 38 implies the plane subgraph $P_{0}+P_{1}+P_{2}+P_{3}$ of $H$ has four facial cycles, each of which is the sum of two paths in $\left\{P_{0}, P_{1}, P_{2}, P_{3}\right\}$. If one of these facial cycles uses two $x_{1}$-edges, then we may assume $P_{0}+P_{1}+P_{2}+P_{3}=H_{34 a}$. Otherwise, we may assume $P_{0}+P_{1}+P_{2}+P_{3}=H_{34 c}$.

Suppose $H$ has a well-fitted $H_{34 a}$. Lemma 44 implies there are ear paths $Q$ and $R$ across $P_{0}+P_{1}$ and $P_{2}+P_{3}$, respectively, which separate $x$ and $y$ (F. 34b). We would like the origin of one of these ear paths to be on $P_{0}+P_{3}$, and the origin of the other to be on $P_{1}+P_{2}$ as shown in Figure 34b. If this is not true, then we may assume the origins of both $Q$ and $R$ are on $P_{1}+P_{2}$. By Lemma 33 we know $H_{34 a}$ has an ear path $S$ with origin on $P_{0}+P_{3}-\{x, y\}$ and terminus on $P_{1}+P_{2}-\{x, y\}$. Furthermore, $S$ is an ear path across either $P_{0}+P_{1}$ or $P_{2}+P_{3}$ which separates $x$ and $y$. Then $Q$ or $R$ can be replaced by $S$. Hence, we can assume $H$ has a well-fitted $H_{34 b}$. Let $s$ and $t$ be the origin and terminus, respectively, of $R$. Since $H_{34 b}-\left(P_{2}[s, y]+P_{3}[x, t]\right)$ is well-fitted to $H$ by Lemma 26, and it is an $H_{29 i}$ bisubdivision, we are done.

Suppose $H$ has a well-fitted $H_{34 c}$. Let $w_{k}$ be the second to last vertex on $P_{k}$ for $k=0,1,2,3$. By Lemma 31 and the comment after it, we may assume $H_{34 c}$ has an ear path $Q_{k}$ with origin $w_{k}$ and a terminus, $t_{k}$, that is not on $P_{k}$ for $k=0,1,2,3$. Each of $Q_{0}$, $Q_{1}, Q_{2}$ and $Q_{3}$ is an ear path across a facial cycle of $H_{34 c}$. Hence, $t_{k}$ is an intermediate vertex of either $Q_{k-1}$ or $Q_{k+1}$ for $k=0,1,2,3$, where addition is modulo 4 . By symmetry, we may assume $t_{0}$ is on $P_{1}$. Suppose $t_{3}$ is on $P_{2}($ F. $34 \mathrm{~d})$. Since $H_{34 d}-\left(w_{0} y+P_{1}\left[x, t_{0}\right]\right)$ is well-fitted to $H$ by Lemma 26, and it is an $H_{29 i}$ bisubdivision, we are done. Therefore, we may assume $t_{3}$ is on $P_{0}$. Similarly, we may assume $t_{1}$ is on $P_{2}$, and $t_{2}$ is on $P_{3}$. Now $H$ has a well-fitted $H_{34 e}$. Then $G$ has a well-fitted $H_{34 f}$. Since $H_{34 f}$ is an $H_{14}$ bisubdivision, either $G$ is an $H_{14}$ or we have Case 1 .

Now that the special subcase is done, we proceed with the rest of this case. $H$ has a well-fitted $H_{35 a}$ by Lemma 50. [Note the names given to the three $(x, y)$-paths of $H_{35 a}$.] Since $G$ is a type 3 augmentation of $H$, there exists an $x_{2}$-edge $e$ which is not on $H_{35 a}$. Furthermore, Lemma 26 implies $H_{35 a}$ is 1-extendible. Hence, we can apply Lemma 32a to $H_{35 a}$, its 2-path $P_{2}$, and $e$. By the comment after Lemma 32 we may assume $H_{35 a}$ has an ear path $Q$ whose first edge is $e$ and whose terminus, $z$, is not an intermediate vertex of $P_{2}$. If $z=y$, then we have the special subcase. For the other two possibilities for $z$, we see $H$ has a well-fitted $H_{35 b}$ or $H_{35 c}$. Since $H_{35 b}$ is an $H_{29 \ell}$ bisubdivision, we may assume $H$ has a well-fitted $H_{35 c}$. 

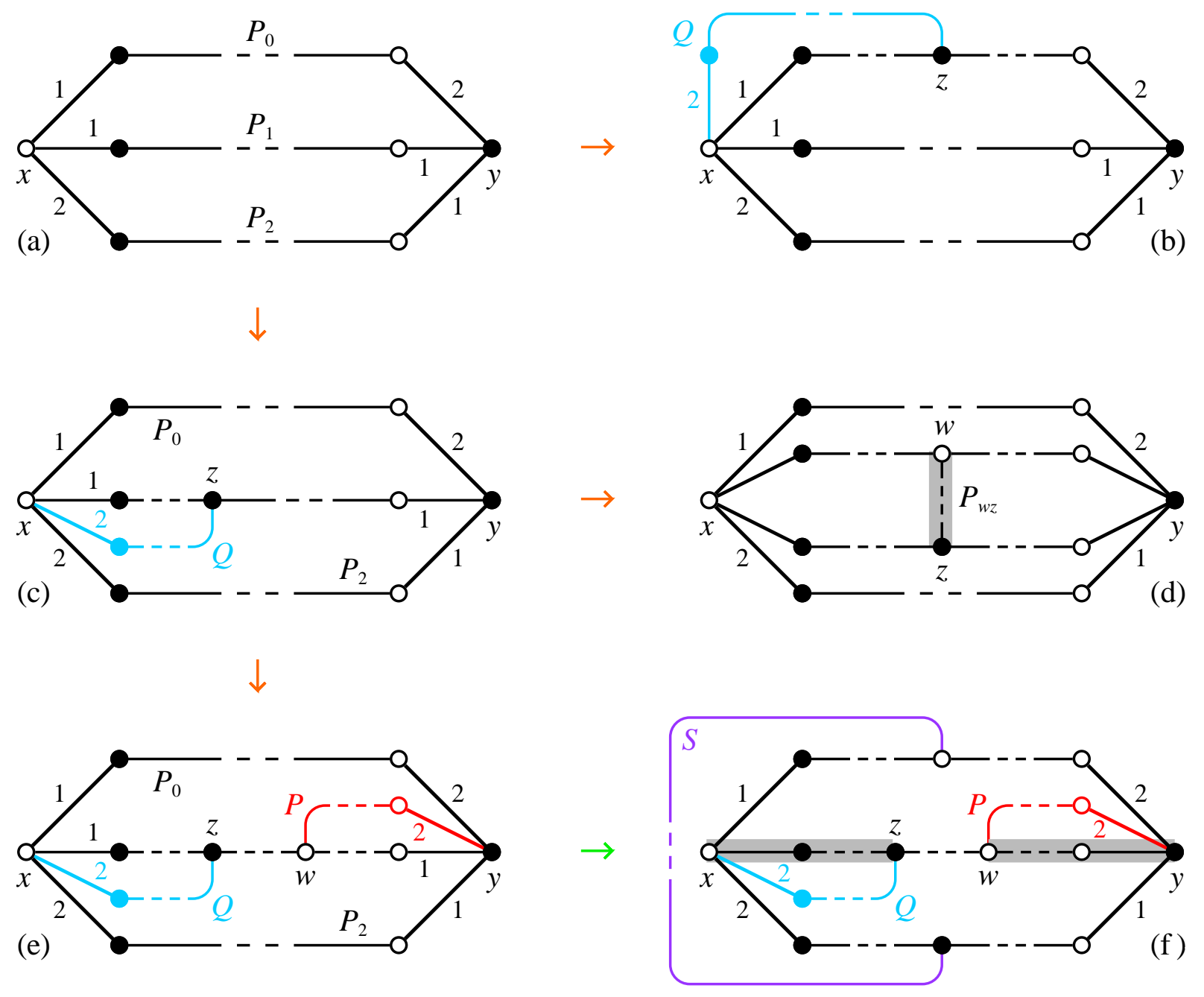

Figure 35: Case 5

Using Lemma 32a again, we may assume $H_{35 c}$ has an ear path $P$ whose last edge is an $y_{2}$-edge and whose origin, $w$, is not an intermediate vertex of $P_{0}$. By analogy with the previous paragraph, we are done if $w$ is on $P_{2}$. If $w$ is an intermediate vertex of $Q$ or $P_{1}[x, z]$, then $H$ has a well-fitted $H_{35 d}$. Then $H_{35 d}-P_{w z}$ is well-fitted to $H$ by Lemma 26; and so we have the special subcase. The only other possibility is that $w$ is on $P_{1}(z, y)$, and then $H$ has a well-fitted $H_{35 e}$. The planar embedding of $H_{35 e}$ might not be exactly as shown in Figure 35e, but $P_{0}+P_{2}$ is a facial cycle of $H_{35 e}$ in any embedding by Theorem 38. By assumption, $x$ and $y$ are not on a common face; and so $x$ and $y$ are separated by some ear path $S$ across $P_{0}+P_{2}$ by Lemma 44. If the origin of $S$ is on $P_{0}$, then $H$ has a well-fitted $H_{35 f}$. Since $H_{35 f}-\left(P_{2}[x, z]+P_{2}[w, y]\right)$ is well-fitted to $H$ by Lemma 26 , and it is an $H_{29 i}$ bisubdivision (with the symmetric roles of $x_{1}$-edges and $x_{2}$-edges interchanged), we are done. The possibility of the origin of $S$ being on $P_{2}$ is handled in a similar fashion, except $P$ and $Q$ are removed instead of $P_{2}[x, z]$ and $P_{2}[w, y]$. 
Case 6. Suppose $G$ is a type 3 augmentation of a plane graph $H$, where $x$ and $y$ are on a facial cycle $C$ of $H$ (F. 36c). Since $G$ is a type 3 augmentation, $x$ and $y$ are nonadjacent. By Theorem 36 there is a planar map $\left\{\rho_{v} \mid v \in V(H)\right\}$ for $H$. Let $\rho_{x}=\left(x z_{1}, \ldots, x z_{m}\right)$ and $\rho_{y}=\left(w_{1} y, \ldots, w_{n} y\right)$. Let $P$ and $Q$ be the two internally disjoint $(x, y)$-paths of $C$. We may assume $x z_{2}$ and $w_{1} y$ are on $Q$, and $x z_{1}$ is on $P$. Then $w_{2} y$ is on $P$ by Theorem 36b. Without loss of generality, $x z_{1}$ is an $x_{1}$-edge and $w_{1} y$ is a $y_{1}$-edge.

Suppose $x z_{2}$ is an $x_{1}$-edge. By the definition of a type 3 augmentation there exist $x_{2}$-edges $x z_{i}$ and $x z_{j}$, where $i$ and $j$ are in $\{3, \ldots, m\}$. If $w_{2} y$ is a $y_{2}$-edge, then $H$ has a well-fitted $H_{36 a}$ by Lemma 45. Since $H_{36 a}$ is an $H_{29 h}$ bisubdivision, we are done. Therefore, we may assume $w_{2} y$ is a $y_{1}$-edge. By the definition of a type 3 augmentation there exists a $y_{2}$-edge $w_{k} y$, where $3 \leq k \leq n$. Then $H$ has a well-fitted $H_{36 d}$ by Lemma 46 . (By symmetry, we may assume Lemma 46 gives us ear paths $R$ and $S$ of $C$ such that $x z_{i}$ and $w_{k} y$ are on $R$, and the terminus, $z$, of $S$ is on $P$.) Since $C$ is a facial cycle of $H$, it is also a facial cycle of $H_{36 d}$. Theorem 38 can then be used to determine the other facial cycles. In particular, $R+Q$ is a facial cycle of $H_{36 d}$. By Lemma 33 there is an ear path $T$ of $H_{36 d}$ with origin on $Q\left[z_{2}, w_{1}\right]$ and terminus on another component of $H_{36 d}-\{x, y\}$. Then $T$ is across either $C$ or $R+Q$. But $T$ can not be across $C$ because $C$ is a facial cycle of $H$. Thus, $T$ is across $R+Q$. Therefore, $H$ has a well-fitted $H_{36 f}$. Since $H_{36 f}-P[x, z]$ is well-fitted to $H$ by Lemma 26 , and it is an $H_{29 i}$ bisubdivision (with the roles of $x_{1}$-edges and $x_{2}$-edges interchanged, and the roles of $y_{1}$-edges and $y_{2}$-edges interchanged), we are done. Thus, we may assume $x z_{2}$ is an $x_{2}$-edge. Similarly, we may assume $w_{2} y$ is a $y_{2}$-edge.

Suppose there exists $k$ in $\{3, \ldots, m-1\}$ such that $x z_{2}, \ldots, x z_{k}$ are $x_{2}$-edges and $x z_{k+1}, \ldots, x z_{m}, x z_{1}$ are $x_{1}$-edges (F. 36e), and there exists $\ell$ in $\{3, \ldots, n-1\}$ such that $w_{2} y, \ldots, w_{\ell} y$ are $y_{2}$-edges and $w_{\ell+1} y, \ldots, w_{n} y, w_{1} y$ are $y_{1}$-edges. Then $G$ is planar as shown in Figure 36g. (This can be rigorously proven by using Theorem 38 and showing that a planar mesh for $H$ can be modified to give a planar mesh for $G$.) Hence, $G \in \mathcal{G}$. If such a $k$ and $\ell$ do not both exist (F. 36b), then we may assume there exists an $x_{1}$-edge $x z_{i}$ and an $x_{2}$-edge $x z_{j}$ such that $3 \leq i<j \leq m$. By Lemma 45, there exist internally disjoint ear paths $R_{P}$ and $R_{Q}$ of $C$ such that $x z_{i}$ and $x z_{j}$ are their first edges, the terminus of $R_{P}$ is an intermediate vertex of $P$, and the terminus of $R_{Q}$ is an intermediate vertex of $Q$. Using the same argument used in the last paragraph of Case 4 , we can show $x z_{i}$ is the first edge of $R_{Q}$, and $x z_{j}$ is the first edge of $R_{P}$, as shown in Figure 36b. Since $H_{36 b}$ is an $H_{29 h}$ bisubdivision, we are done.

Case 7. Suppose $G$ is a type 4 augmentation of a plane graph $H$. By Theorem $36, H$ has a planar map $\left\{\rho_{v} \mid v \in V(H)\right\}$. Let $\rho_{x}=\left(x y, x z_{1}, \ldots, x z_{m}\right)$ and $\rho_{y}=\left(x y, w_{1} y, \ldots, w_{n} y\right)$. We start with three observations.

First, suppose $H_{37 a}$ is a plane subgraph of $H$. Note the vertex names, and that $P$ and $Q$ are $(x, y)$-paths. We will show that $1 \leq i<j \leq m$ implies $1 \leq k<\ell \leq n$. By Theorem 37 there is a planar map $\left\{\rho_{v}^{\prime} \mid v \in V\left(H_{37 a}\right)\right\}$ for $H_{37 a}$ such that $\rho_{v}^{\prime}$ is a subpermutation of $\rho_{v}$ for every $v$ in $V\left(H_{37 a}\right)$. Then $1 \leq i<j \leq m$ implies $\rho_{x}^{\prime}=\left(x y, x z_{i}, x z_{j}\right)$. Theorem 38 can be used to show $Q+x y$ is a facial cycle of $H_{37 a}$. Now we apply Theorem $36 \mathrm{~b}$ to $Q+x y$. Since $\rho_{x}^{\prime}\left(x z_{j}\right)=x y$, we have $\rho_{y}^{\prime}(x y)=w_{k} y$. Hence, $\rho_{y}^{\prime}=\left(x y, w_{k} y, w_{\ell} y\right)$. Finally, since $\rho_{y}^{\prime}$ is a subpermutation of $\rho_{y}$, we have $1 \leq k<\ell \leq n$. 


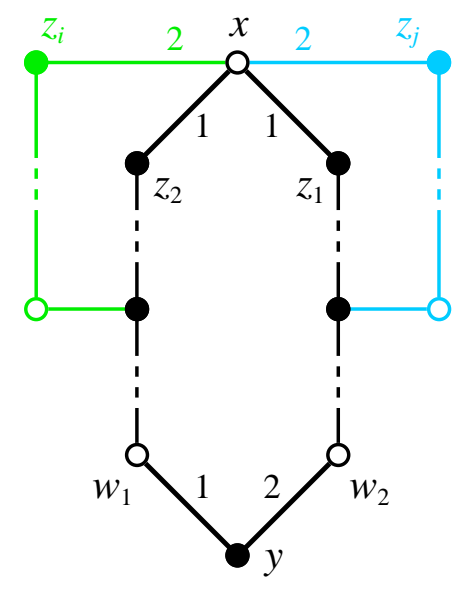

(a)

(b)
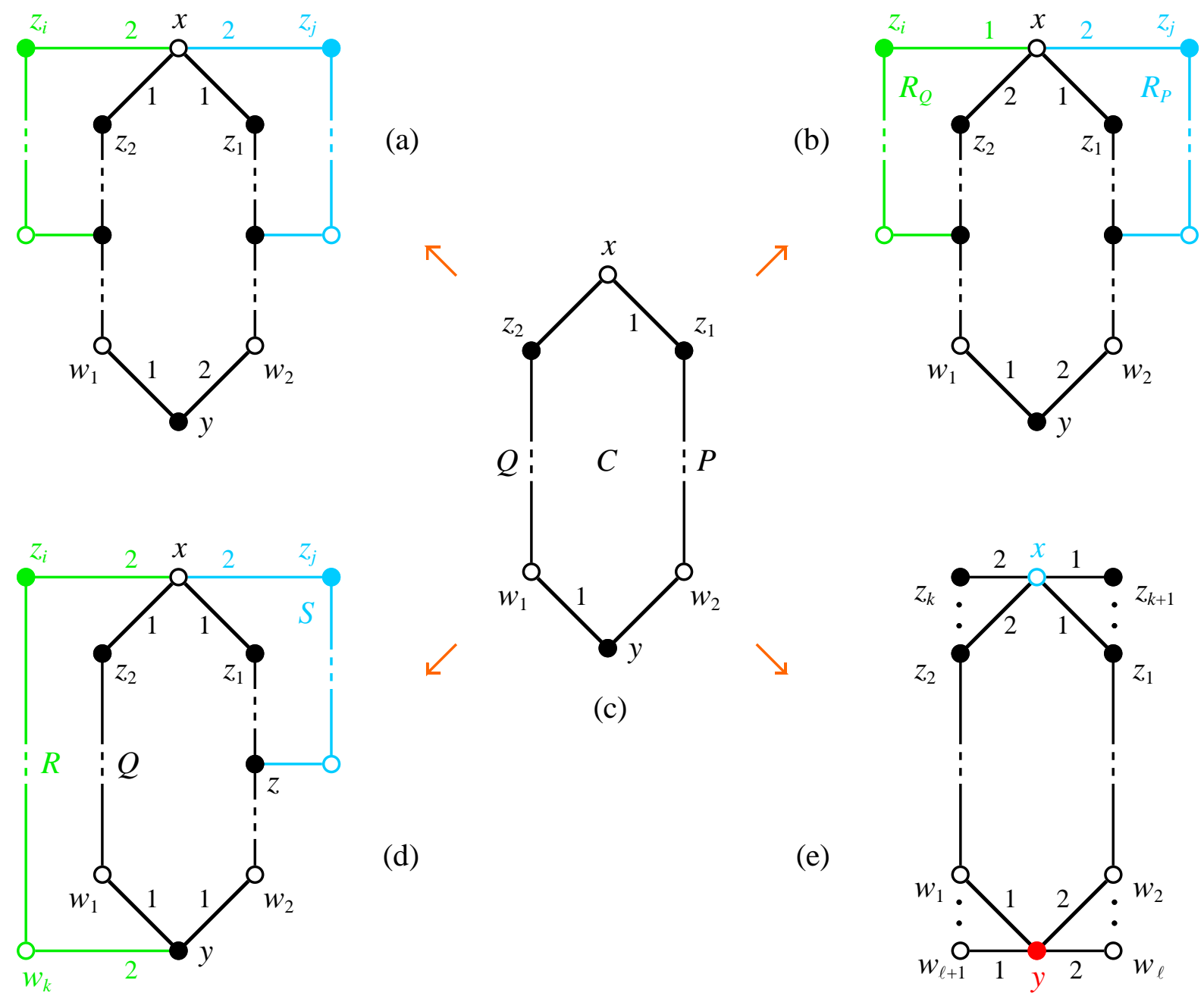

(d)

(c)

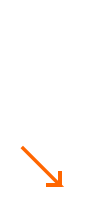

(e)
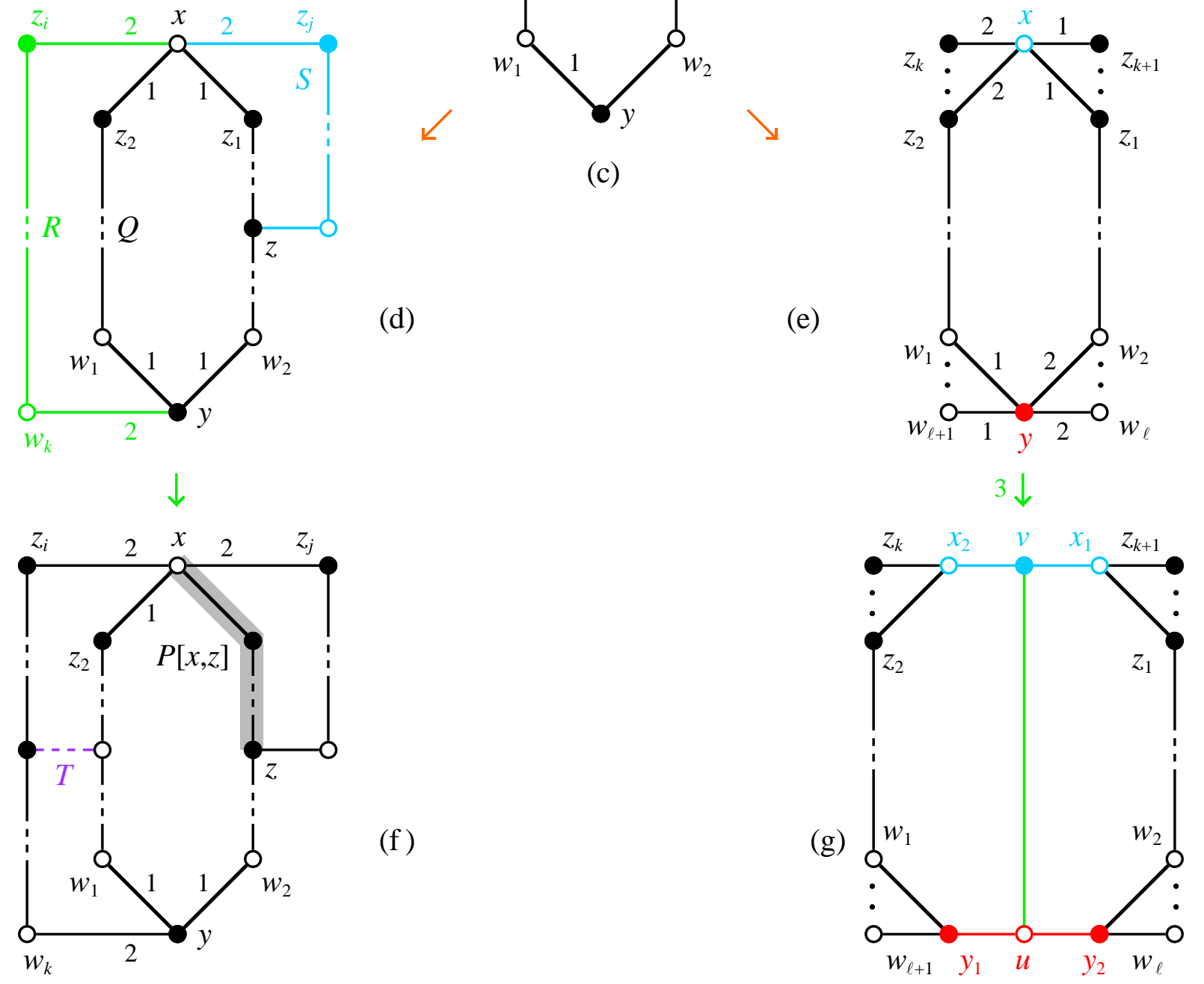

Figure 36: Case 6 


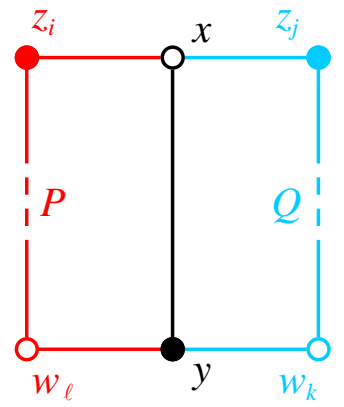

(a)

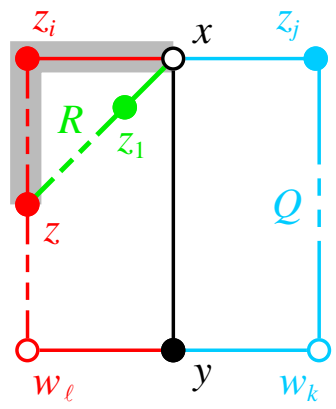

(b)

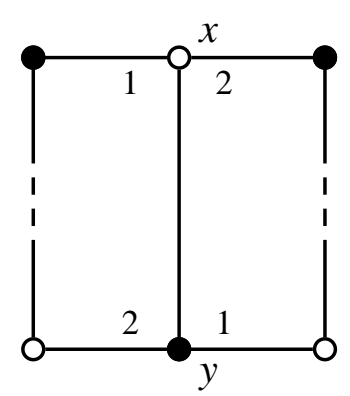

(c)

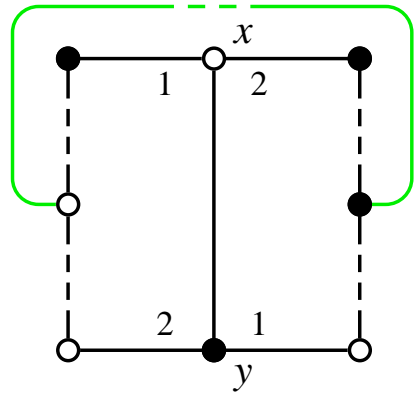

(d)

Figure 37: Case 7

Second, we will show that if $H$ has a well-fitted $H_{37 a}$, then we may assume $i=1$. Suppose $2 \leq i<j \leq m$ and $1 \leq k<l \leq n$. By Lemma 29 there is an ear path $R$ of $H_{37 a}$ with first edge $x z_{1}$ and terminus, $z$, not equal to $y$ (F. 37b). If $z$ is on $Q$, then $P$ and $R+Q[z, y]$ contradict the observation of the previous paragraph. Hence, $z$ is on $P$. Then $H_{37 b}-P[x, z]$ is well-fitted to $H$ by Lemma 26. Similarly, we may assume $j=m$, $k=1$, and $\ell=n$ in $H_{37 a}$.

Third, we will show that if $H$ has a well-fitted $H_{37 c}$, then $H$ has a well-fitted $K_{3,3}$ bisubdivision. By Lemma 33 there is an ear path of $H_{37 c}$ whose ends are in different components of $H_{37 c}-\{x, y\}$. Without loss of generality, $H$ then has a well-fitted $H_{37 d}$. Since $H_{37 d}$ is an $H_{29 a}$ bisubdivision, we are done. Now that we have made the three observations we proceed with the rest of this case.

Suppose $x z_{1}$ is an $x_{1}$-edge and $w_{n} y$ is a $y_{2}$-edge. Theorem 36 can be used to show $w_{n} y x z_{1}$ is a subgraph of some facial cycle $C$ of $H$ (F. 38a). Then $C$ is well-fitted to $H$ by Lemma 42, and $H-V(C)$ is 1-extendible by Corollary 43. By the definition of a type 4 augmentation, there exists an $x_{2}$-edge $x z_{j}$ and a $y_{1}$-edge $w_{k} y$. By Lemma 34 there is an ear path $Q$ of $C$ using $x z_{j}$ and $w_{k} y$. Then $C+Q$ is a well-fitted $H_{37 c}$ subgraph of $H$. Hence, $G$ has a well-fitted $K_{3,3}$ bisubdivision by the third observation.

Suppose $x z_{1}$ is an $x_{1}$-edge and $x z_{m}$ is an $x_{2}$-edge. By the previous paragraph we may assume $w_{n} y$ is a $y_{1}$-edge, and by a very similar argument we may assume $w_{1} y$ is a $y_{2}$-edge (F. 38b). Suppose there exists $k$ in $\{1, \ldots, m-2\}$ such that $x z_{1}, \ldots, x z_{k}$ are $x_{1}$-edges and $x z_{k+1}, \ldots, x z_{m}$ are $x_{2}$-edges, and there exists $\ell$ in $\{2, \ldots, n-1\}$ such that $w_{1} y, \ldots, w_{\ell} y$ are $y_{2}$-edges and $w_{\ell+1} y, \ldots, w_{n} y$ are $y_{1}$-edges $(\mathrm{F} .38 \mathrm{e}$ ). Then $G$ is planar as shown in Figure 38d. (This can be rigorously proven by using Theorem 38 and showing that a planar mesh for $H$ can be modified to give a planar mesh for $G$.) Hence, $G \in \mathcal{G}$. If such a $k$ and $\ell$ do not both exist (F. 38c), then we may assume there exists an $x_{2}$-edge $x z_{i}$ and an $x_{1}$-edge $x z_{j}$ such that $2 \leq i<j \leq m-1$. By Lemma 51 , there are internally disjoint $(x, y)$-paths $P$ and $Q$ (where $P \neq x y \neq Q$ ) such that $P+Q+x y$ is a well-fitted subgraph of $H, x z_{i}$ is on $P$, and $x z_{j}$ is on $Q$ (F. 38f). We may assume $w_{n} y$ and $w_{1} y$ are the last edges of $P$ and $Q$ by the second observation. By the first observation $w_{n} y$ is on $P$ and $w_{1} y$ is on $Q$. Hence, $G$ has a well-fitted $K_{3,3}$ bisubdivision by the third observation. 
(a)

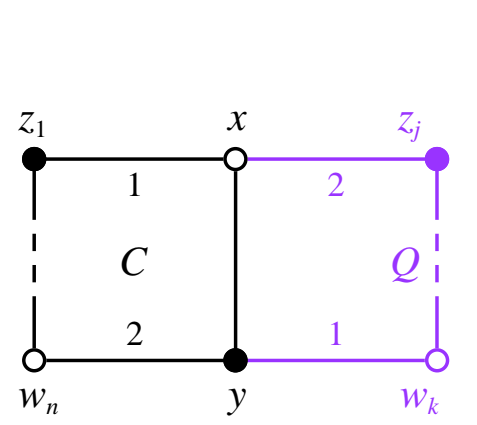

(b)

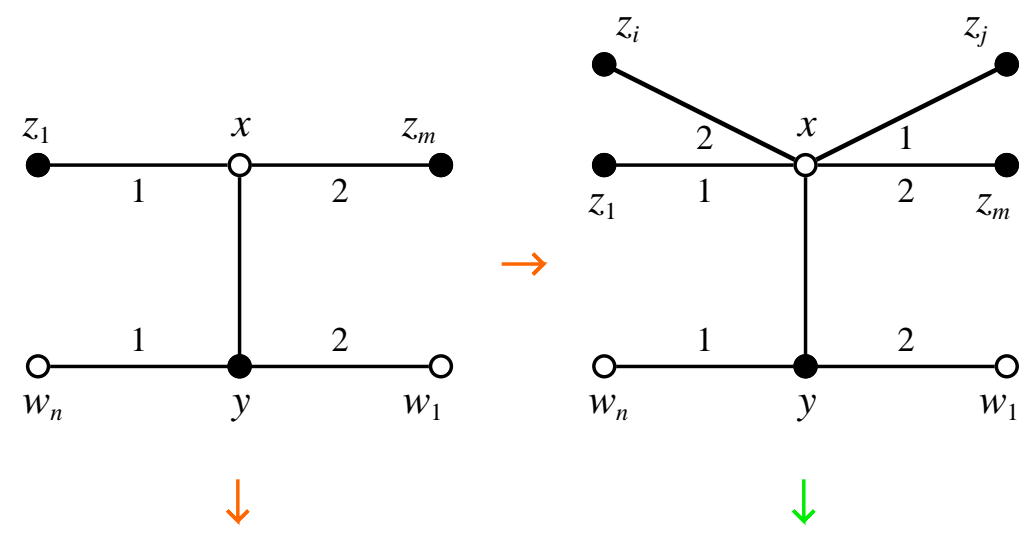

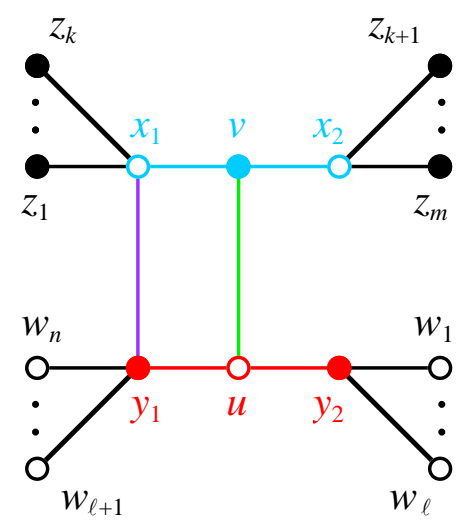

(d)

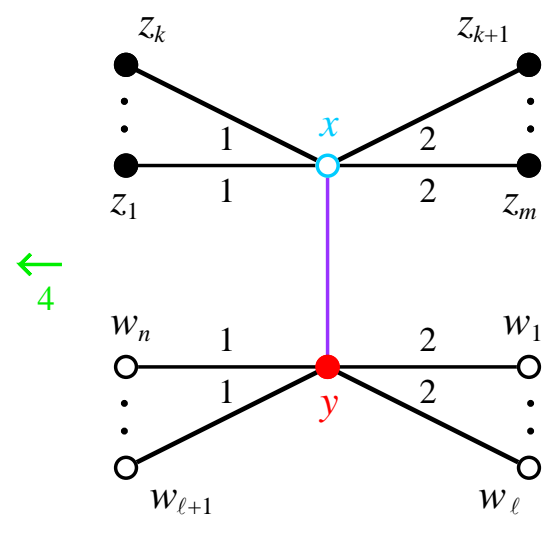

(e)

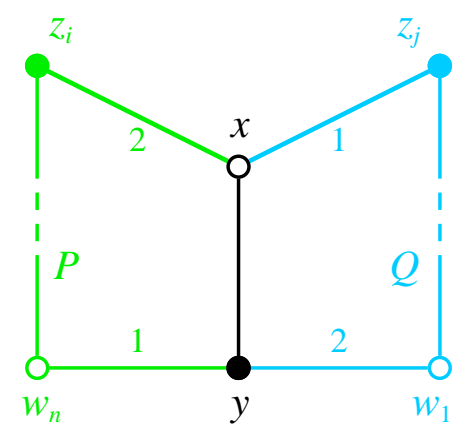

(f)

Figure 38: Case 7

By the previous paragraph we may assume $x z_{1}$ and $x z_{m}$ are either both $x_{1}$-edges or both $x_{2}$-edges. Suppose they are both $x_{1}$-edges. By the definition of a type 4 augmentation there exist $x_{2}$-edges $x z_{i}$ and $x z_{j}$, where $2 \leq i<j \leq n-1$ (F. 39a). Then $H$ has a wellfitted $H_{39 b}$ by Lemma 51. [Without loss of generality, a $y_{2}$-edge is the last edge of the $(x, y)$-path using $x z_{i}$.] Using the second observation we can "replace" $i$ by 1 to obtain a well-fitted $H_{\underline{37}}$ subgraph of $H$. Hence, $G$ has a well-fitted $K_{3,3}$ bisubdivision by the third observation. Similarly, we can complete this case if $x z_{1}$ and $x z_{m}$ are both $x_{2}$-edges, and there exist at least two $x_{1}$-edges. But there may be only one $x_{1}$-edge for a type 4 augmentation. Therefore, we may assume $x z_{1}$ and $x z_{m}$ are $x_{2}$-edges and there is a unique $x_{1}$-edge $x z_{i}$, where $2 \leq i \leq m-1$ (F. 39d). Similarly, we may assume $w_{1} y$ and $w_{m} y$ are $y_{2}$-edges and there is a unique $y_{1}$-edge $w_{k} y$, where $2 \leq k \leq n-1$.

Let $K=H-\left\{z_{i}, x, y, w_{k}\right\}$. With the aid of Figures $39 \mathrm{~d}$ and $39 \mathrm{e}$ we see that the components of $G-\left\{z_{i}, x_{2}, y_{2}, w_{k}\right\}$ are the 4-cycle $u v x_{1} y_{1} u$ and the components of $K$. Hence, if $K$ has at least two components, then $G-\left\{z_{i}, x_{2}, y_{2}, w_{k}\right\}$ has at least three components; and so we have the Special Case. Thus, we may assume $K$ is connected. 


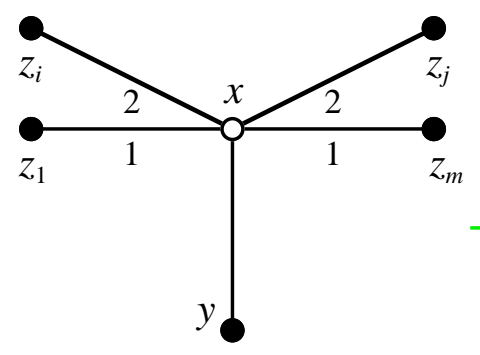

(a)
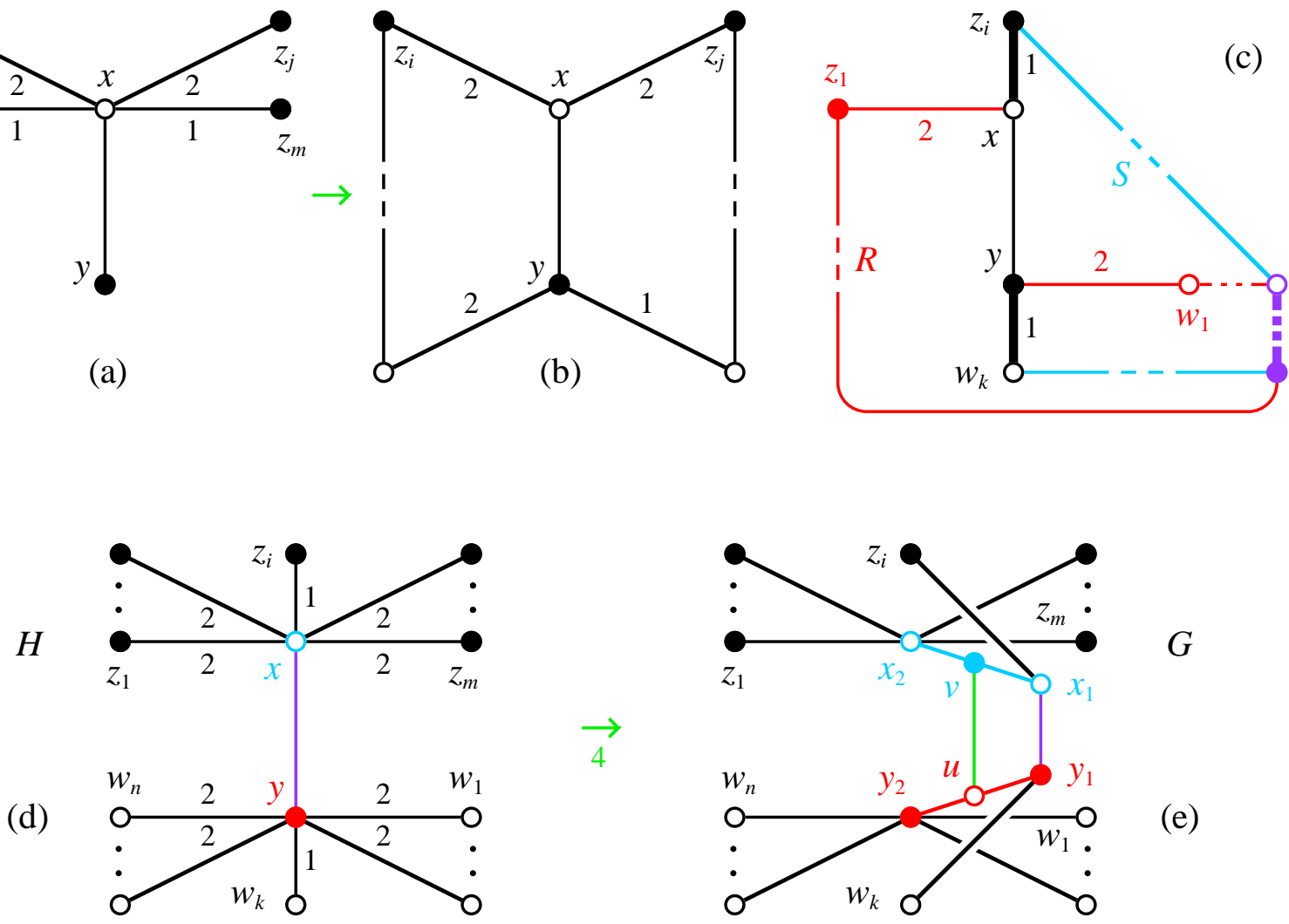

$G$

(e)

Figure 39: Case 7

Suppose $w z$ is an arbitrary edge of $K$. Since $H$ is a brace, Corollary 17 implies $H-\{w, z\}$ is 1-extendible. Then $H-\{w, z\}$ has perfect matchings $M_{x z_{i}}$ and $M_{w_{k} y}$ containing $x z_{i}$ and $w_{k} y$, respectively. Suppose $H\left[M_{x z_{i}} \cup M_{w_{k} y}\right]$ has an alternating cycle $C$ using $x z_{i}$ and $w_{k} y$. Each $(x, y)$-path of $C$ has odd length; and so each is well-fitted to either $M_{x z_{i}}$ or $M_{w_{k} y}$. Hence, $x z_{i}$ and $w_{k} y$ are on different $(x, y)$-paths of $C$. It follows that $C+x y$ is a well-fitted $H_{37 c}$ subgraph of $H$; and so we are done by the third observation. Therefore, we may assume $x z_{i}$ and $w_{k} y$ are on different components of $H\left[M_{x z_{i}} \cup M_{w_{k} y}\right]$. It is then easy to obtain a perfect matching $M$ of $H-\{w, z\}$ containing $x z_{i}$ and $w_{k} y$. Then $\left(M-\left\{x z_{i}, w_{k} y\right\}\right) \cup\{w z\}$ is a perfect matching of $K$ which includes $w z$. Thus, we may assume for every edge $e$ of $K$, some perfect matching of $K$ contains $e$.

Since $H$ is a planar brace, $\nu(H) \geq 8$; and so $\nu(K) \geq 4$. Then the last sentences of the previous two paragraphs imply $K$ is 1-extendible. Now consider the well-fitted subgraph $z_{i} x y w_{k}$ of $H$ (F. 39c). Since $K$ is 1-extendible, we can use Lemma 34 to obtain an ear path $R$ of $z_{i} x y w_{k}$ using $x z_{1}$ and $w_{1} y$, and an ear path $S$ of $z_{i} x y w_{k}$ with ends $w_{k}$ and $z_{i}$. Using Lemma 27 we may assume either $R$ and $S$ are disjoint, or $R \cap S$ is a path. But if $R$ and $S$ are disjoint, then we contradict the first observation. Therefore, $R \cap S$ is a path, and $H_{39 c}$ is a well-fitted subgraph of $H$. Since $H_{39 c}-(R \cap S)$ is well-fitted to $H$ by Lemma 26 , and it is an $H_{\underline{37}}$, we are done by the third observation. 
Case 8. Suppose $G$ is a type 3 augmentation of a graph $H$ in $\mathcal{G}$ which is a 4 -cycle sum of $H_{1}, \ldots, H_{n}$ at $C=a b c d a$, where $n \geq 3$.

Suppose $x$ and $y$ are not on $C$. We may assume $x$ is in $V\left(H_{1}\right)-V(C)$. If $y$ is in $V\left(H_{1}\right)-V(C)$, then $G-\{a, b, c, d\}$ has $n$ components; and so we have the Special Case. Hence, we may assume $y$ is in $V\left(H_{2}\right)-V(C)$. We now apply Lemma 54 to $H_{1}$ and $H_{2}$. By symmetry, we may assume $H_{1}$ has an $H_{40 a}$ subgraph which is well-fitted to a perfect matching $M_{1}$ of $H_{1}$, and $H_{2}$ has an $H_{40 b}$ subgraph which is well-fitted to a perfect matching $M_{2}$ of $H_{2}$. Lemmas 47 and 26 imply $H_{3}$ has an $H_{40 c}$ subgraph which is well-fitted to a perfect matching $M_{3}$ of $H_{3} . H_{40 a}$ and $H_{40 b}$ have perfect matchings containing $a b$ and $c d$, and $H_{40 c}$ has a perfect matching which contains no edge of $C$. Hence, we may assume $M_{1}$ and $M_{2}$ contain $a b$ and $c d$, and $M_{3}$ contains no edge of $C$. If $4 \leq k \leq n$, then the brace $H_{k}$ has a perfect matching $M_{k}$ containing $a b$ and $c d$. Then $\left(H_{40 a}+H_{40 b}+H_{40 c}\right)-E(C)$ is an $H_{40 j}$ subgraph of $H$ which is well-fitted to the perfect matching $\cup_{k=1}^{n} M_{i}-\{a b, c d\}$. Since $H_{40 j}$ is an $H_{29 j}$ bisubdivision, we are done.

Suppose $x$ is on $C$ and $y$ is not on $C$. We may assume $x=a$ and $y$ is in $V\left(H_{3}\right)-V(C)$. By applying Corollary 18 to $H_{k}$, we know there is at least one edge of $H_{k}-E(C)$ incident with $x$ for $k=1,2$. Furthermore, if all the edges of $\left(H_{1}+H_{2}\right)-E(C)$ incident with $x$ are $x_{i}$-edges for some $i$ in $\{1,2\}$, then $G-\left\{x_{i}, b, c, d\right\}$ has at least three components; and so we have the Special Case. Therefore, we may assume there exists an $x_{k}$-edge $x z_{k}$ of $H_{k}-E(C)$ for $k=1,2$. As in the previous paragraph, we can use Lemmas 26, 47, and 54 to show that $H_{1}, H_{2}$, and $H_{3}$ have well-fitted $H_{40 d}, H_{40 e}$, and $H_{40 f}$ subgraphs, respectively, and furthermore, $H_{40 k}=\left(H_{40 d}+H_{40 e}+H_{40 f}\right)-E(C)$ is well-fitted to $H$. Since $H_{40 k}$ is an $H_{29 k}$ bisubdivision, we are done.

Suppose $x$ and $y$ are on $C$. We may assume $x=a$ and $y=b$. Suppose $E\left(H_{k}\right)-E(C)$ contains an $x_{k}$-edge and a $y_{k}$-edge for $k=1,2$, and $E\left(H_{3}\right)-E(C)$ contains an $x_{1}$-edge and a $y_{2}$-edge (F. 40ghi). As before, we can show $H_{40 \ell}=\left(H_{40 g}+H_{40 h}+H_{40 i}\right)-E(C)$ is well-fitted to $H$. Since $H_{40 \ell}$ is an $H_{29 i}$ bisubdivision, we are done.

Finally, we show that if the situation of the previous paragraph does not occur when $x=a$ and $y=b$, then we have the Special Case. To do this we construct an auxiliary bipartite graph $K$. The colour classes of $K$ are $A=\{(1,1),(1,2),(2,1),(2,2)\}$ and $B=\left\{H_{1}, \ldots, H_{n}\right\}$. Vertices $(i, j)$ and $H_{k}$ are adjacent if and only if there is an $x_{i}$-edge and a $y_{j}$-edge in $E\left(H_{k}\right)-E(C)$, for $1 \leq i, j \leq 2$ and $1 \leq k \leq n$. If $K$ has a matching of size three, we have the situation of the previous paragraph. (There may be a reversal of the symmetric roles of $x_{1}$-edges and $x_{2}$-edges, or of $y_{1}$-edges and $y_{2}$-edges. There may also be a permutation of $B$.) Suppose $K$ does not have such a matching. Then there is a subset $S$ of $A$ such that $|N(S)| \leq|S|-2$. (This is proven by applying Theorem 2 to the graph obtained from $K$ by adding a new vertex adjacent to all vertices in $A$, and observing that the new graph has a matching saturating all vertices in $A$ if and only if $K$ has a matching of size three.) We have $2 \leq|N(S)|+2 \leq|S| \leq|A|=4$.

Suppose $|S|=4$, that is, $S=A$. Let $k$ be in $\{1, \ldots, n\} . H_{k}$ has minimum degree at least three by Corollary 18; and so $x$ and $y$ are incident with edges in $E\left(H_{k}\right)-E(C)$. Then the vertex $H_{k}$ of $K$ is adjacent to some vertex in $A$. Hence, $N(S)=N(A)=B$. But then $3 \leq n=|B|=|N(S)| \leq|S|-2=2$, and we have a contradiction. 

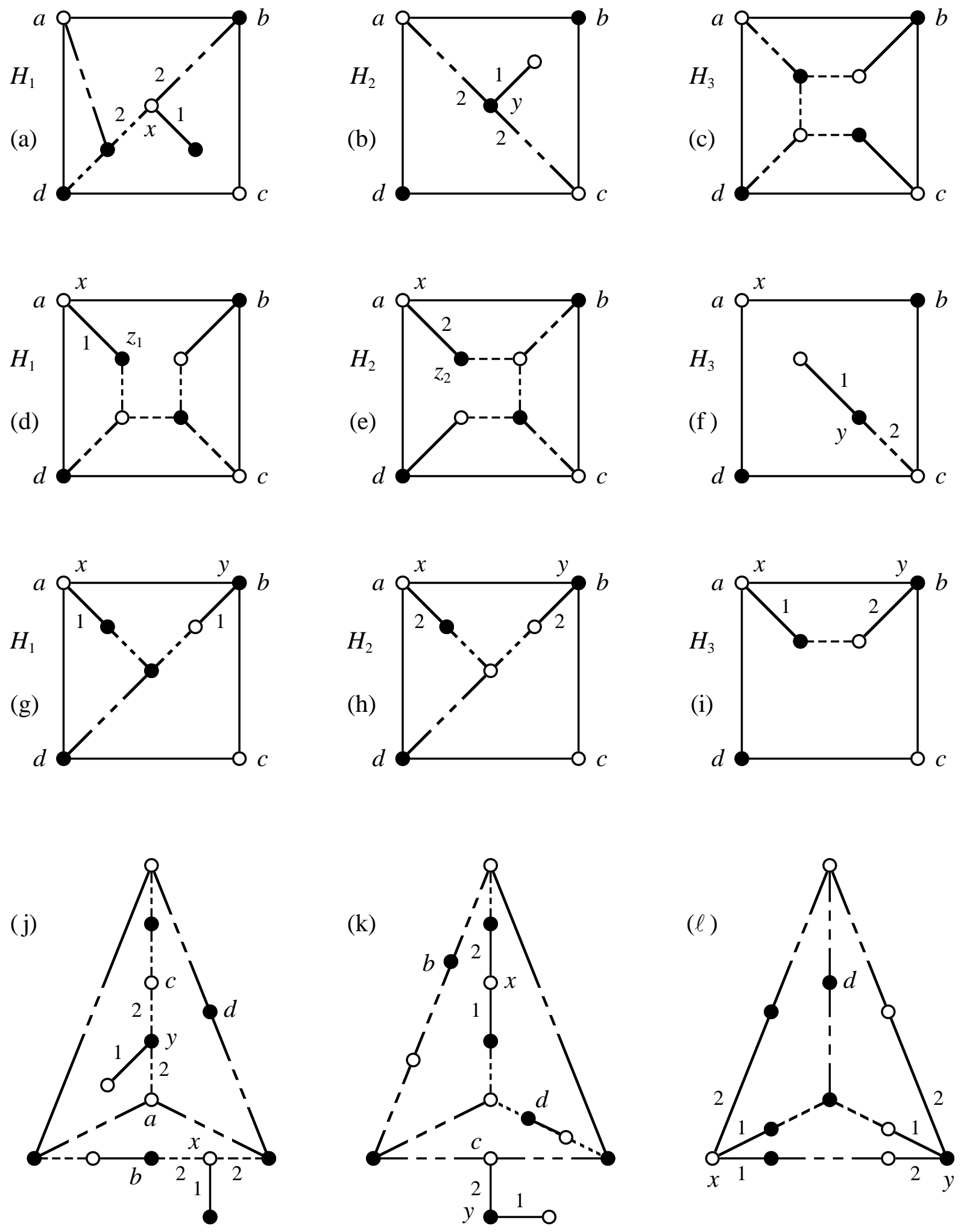

Figure 40: Case 8 
Suppose $|S|=3$. Then $|N(S)| \leq|S|-2=1$ and $S=A-\{(i, j)\}$ for some $i$ and $j$ in $\{1,2\}$. Without loss of generality, $N(S) \subset\left\{H_{n}\right\}$. Then $H_{1}$ and $H_{2}$ are only adjacent to $(i, j)$ in $K$. Hence, $H_{k}-E(C)$ has only $x_{i}$-edges incident with $x$ and $y_{j}$-edges incident with $y$, for $k=1,2$. Then $G-\left\{x_{i}, y_{j}, c, d\right\}$ has at least three components; and so we have the Special Case.

Suppose $|S|=2$. Hence, $|N(S)| \leq|S|-2=0$. Then $N(A-S)=B$ because every vertex in $B$ is adjacent to some vertex in $A$. We may assume $(2,2)$ is in $A-S$. Suppose $A-S=\{(1,1),(2,2)\}$. If some vertex $H_{k}$ in $B$ is adjacent to both $(1,1)$ and $(2,2)$, then $H_{k}$ is also adjacent to $(1,2)$ and $(2,1)$ by the definition of adjacency in $K$. But then $N(S) \neq \emptyset$. Thus, every vertex in $B$ is adjacent to either $(1,1)$ or $(2,2)$. We may assume two vertices in $B$ are only adjacent to $(1,1)$. Then $G-\left\{x_{1}, y_{1}, c, d\right\}$ has at least three components; and so we have the Special Case. Therefore, we may assume $A-S=\{(2,1),(2,2)\}$. Then $a b$ and $a d$ are the only possible $x_{1}$-edges of $H$. But since $G$ is a type 3 augmentation of $H$, there are at least two $x_{1}$-edges, and $a b$ (that is, $x y$ ) is not an edge of $H$. We have a contradiction.

Case 9. Suppose $G$ is a type 4 augmentation of a graph $H$ in $\mathcal{G}$ which is a 4 -cycle sum of $H_{1}, \ldots, H_{n}$ at $C=a b c d a$, where $n \geq 3$. This case is the same as Case 8 up until the last paragraph of Case 8. This paragraph must be modified for the present case because $x_{1}$ and $x_{2}$ (and $y_{1}$ and $y_{2}$ ) do not have symmetric roles for type 4 augmentations as they do for type 3 augmentations.

In the last paragraph of Case 8 we are assuming the auxiliary graph $K$ does not have a matching of size three because there is a subset $S$ of $A$ such that $|S|=2$ and $N(S)=\emptyset$. As in Case 8, we can show $N(A-S)=B$, and $A-S$ is not $\{(1,1),(2,2)\}$. Similarly, we can show $A-S$ is not $\{(1,2),(2,1)\}$. Thus, $A-S$ is $\{(i, 1),(i, 2)\}$ or $\{(1, j),(2, j)\}$ for some $i$ or $j$ in $\{1,2\}$. Using the symmetry between the roles of $x$ and $y$ in a type 4 augmentation, we may assume $A-S=\{(i, 1),(i, 2)\}$ for some $i$ in $\{1,2\}$. Thus, $\cup_{k=1}^{n}\left[E\left(H_{k}\right)-E(C)\right]$ contains only $x_{i}$-edges by the definition of $K$.

Suppose $i=1$. Then $a b$ and $a d$ are the only possible $x_{2}$-edges of $H$. But since $G$ is a type 4 augmentation of $H$, there are at least two $x_{2}$-edges, and $a b$ (that is, $x y$ ) is not by definition an $x_{2}$-edge. We have a contradiction. Thus, $i=2$ and $A-S=\{(2,1),(2,2)\}$.
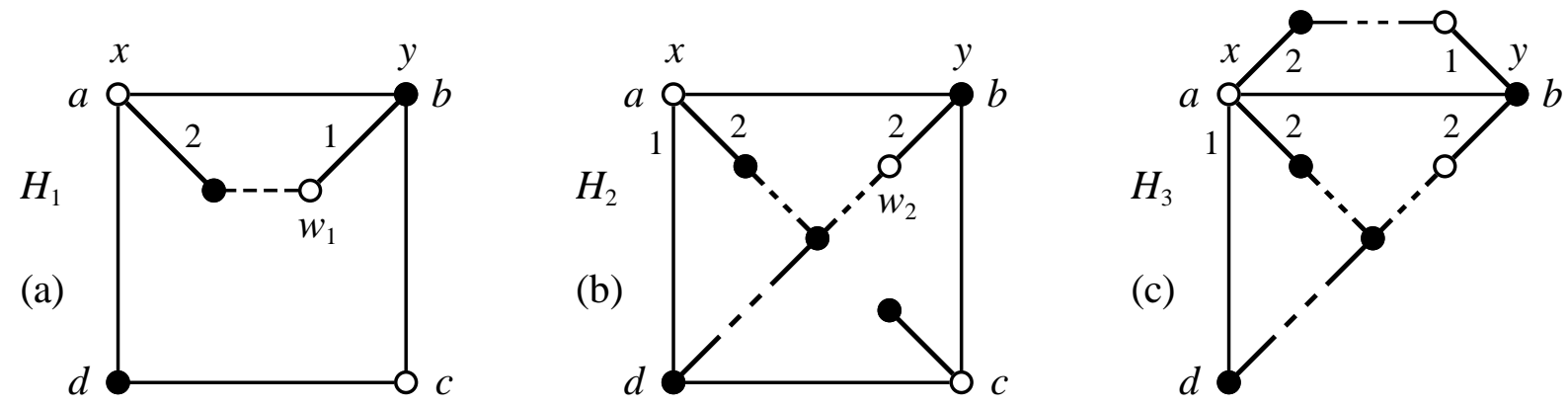

Figure 41: Case 9 

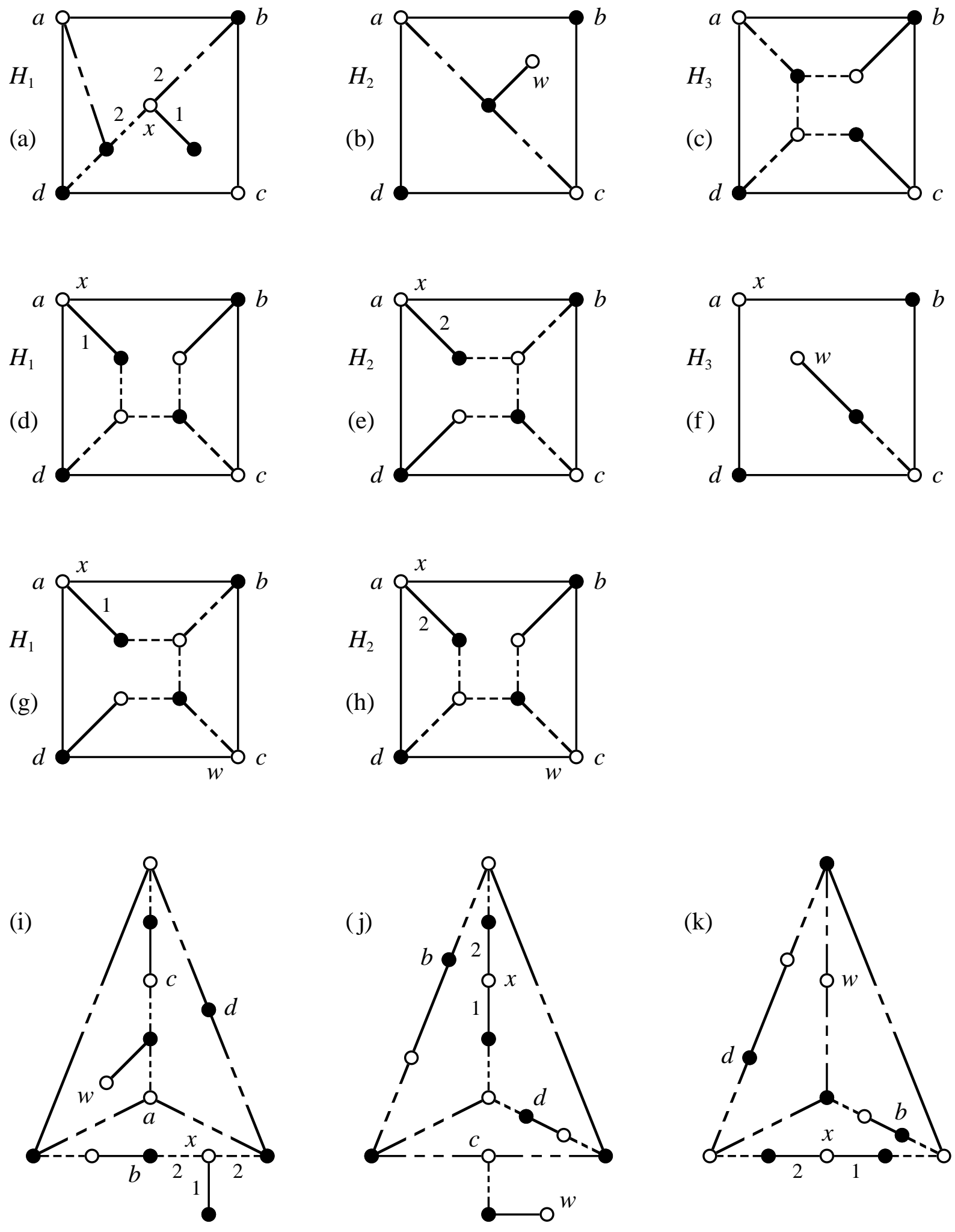

Figure 42: Case 10 
If all the edges of $\left(H_{1}+H_{2}\right)-E(C)$ incident with $y$ are $y_{j}$-edges for some $j$ in $\{1,2\}$, then $G-\left\{x_{2}, y_{j}, c, d\right\}$ has at least three components; and so we have the Special Case. Thus, we may assume there is a $y_{k}$-edge $w_{k} y$ of $H_{k}-E(C)$ for $k=1,2$. We use $H_{41 a}$ and $H_{41 b}$ to show $H$ has a well-fitted $H_{41 c}$. Since $H_{41 c}$ is an $H_{29 b}$ bisubdivision, we are done.

Case 10. Suppose $G$ is a type 2 augmentation of a graph $H$ in $\mathcal{G}$ which is a 4-cycle sum of $H_{1}, \ldots, H_{n}$ at $C=a b c d a$, where $n \geq 3$. We will only give an outline for this case because it is similar to Case 8 .

Suppose $x$ is not on $C$. We may then assume $x$ is in $V\left(H_{1}\right)-V(C)$. If $w$ is in $V\left(H_{1}\right)$, then $G-\{a, b, c, d\}$ has $n$ components; and so we have the Special Case. Hence, we may assume $w \in V\left(H_{2}\right)-V(C)$. We use $H_{42 a}, H_{42 b}$, and $H_{42 c}$ to show $H$ has a well-fitted $H_{42 i}$. Since $H_{42 i}$ is an $H_{29 f}$ bisubdivision, we are done.

Suppose $x$ is on $C$ and $w$ is not on $C$. We may assume $x=a$ and $w$ is in $V\left(H_{3}\right)-V(C)$. If all the edges of $\left(H_{1}+H_{2}\right)-E(C)$ incident with $x$ are $x_{i}$-edges for some $i$ in $\{1,2\}$, then $G-\left\{x_{i}, b, c, d\right\}$ has at least three components; and so we have the Special Case. Thus, we may assume there is an $x_{k}$-edge $x z_{k}$ of $H_{k}-E(C)$ for $k=1,2$. We use $H_{42 d}, H_{42 e}$, and $H_{42 f}$ to show $H$ has a well-fitted $H_{42 j}$. Since $H_{42 j}$ is an $H_{29 e}$ bisubdivision, we are done.

Suppose $x$ and $w$ are on $C$. We may assume $x=a$ and $w=c$. As in the previous paragraph we may assume there is an $x_{k}$-edge $x z_{k}$ of $H_{k}-E(C)$ for $k=1,2$. We use $H_{42 g}$ and $H_{42 h}$ to show $H$ has a well-fitted $H_{42 k}$. Since $H_{42 k}$ is an $H_{29 e}$ bisubdivision, we are done.

Case 11. Suppose $G$ is a type 1 augmentation of a graph $H$ in $\mathcal{G}$ which is a 4-cycle sum of $H_{1}, \ldots, H_{n}$ at $C=a b c d a$, where $n \geq 3$. We will only give an outline for this case because it is similar to Case 8 . If $x$ and $y$ are in $V\left(H_{k}\right)$, where $1 \leq k \leq n$, then we have the Special Case because $G-\{a, b, c, d\}$ has $n$ components. Thus we may assume $x \in V\left(H_{1}\right)-V(C)$ and $y \in V\left(H_{2}\right)-V(C)$. In Figure 43 we show $H$ has a well-fitted $H_{43 d}$. Then $H_{43 d}+x y$ is a $K_{3,3}$ bisubdivision which is well-fitted to $G=H+x y$.

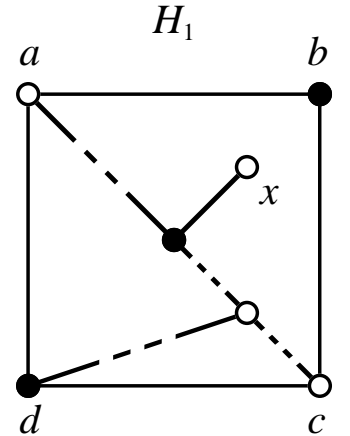

(a)

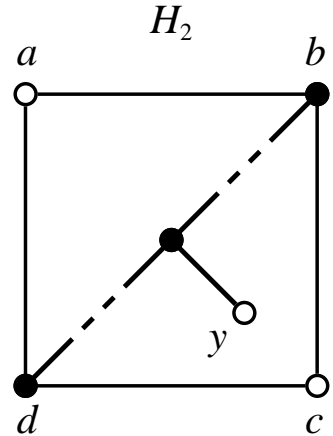

(b)

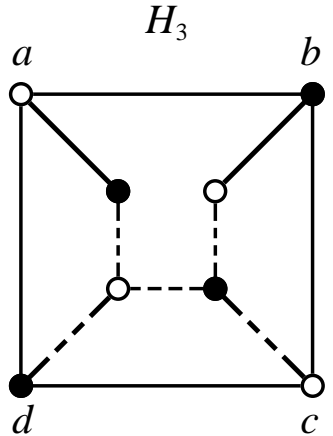

(c)

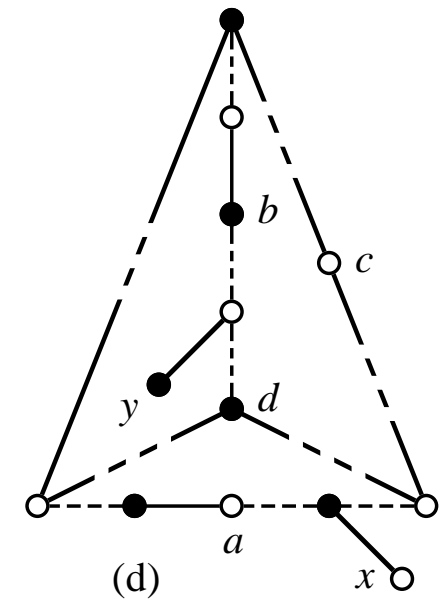

(d)

Figure 43: Case 11 
Main Theorem. The following statements are equivalent for a bipartite graph $G$.

1) $G$ is a brace which has an unbalanced $\{-1,1\}$-edge weighting.

2) $G$ is a brace which does not have a well-fitted $K_{3,3}$ bisubdivision.

3) $G$ is in $\mathcal{G}$.

Proof. Theorem 12 (in the "easy" direction) shows that (1) implies (2). Theorem 56 shows that (2) implies (3). Theorem 55 shows that (3) implies (1).

\section{Obstructions}

Brualdi and Shader [5] have shown that the results of Little (Theorem 12) and Seymour and Thomassen (Theorem 11) can be derived from one another. In this section we combine their proof with the Main Theorem to prove Theorems 11 and 12 .

The set $\left\{M_{6}, M_{10}, M_{14}, \ldots\right\}$ of Möbius ladders is defined in Figure 22. Suppose $n \geq 2$. An edge of $M_{4 n+2}$ which is on two 4-cycles is called a rung of $M_{4 n+2}$. The rungs of a $M_{4 n+2}$ bisubdivision are its 2-paths which have replaced the rungs of $M_{4 n+2}$.

Lemma 57 (F. 44) Suppose $G$ is a bipartite graph with a perfect matching $M$ and a well-fitted $K_{3,3}$ bisubdivision $K$. (Note that $K$ is not necessarily well-fitted to $M$.) Then $G$ has an $M_{4 n+2}$ bisubdivision $L$ which is well-fitted to $M$ for some $n \geq 1$. Furthermore, the rungs of $L$ are well-fitted to $M$ when $n \geq 2$.

Proof. Choose a minimal subgraph $H$ of $G$ such that $M \subset E(H)$ and $H$ has a well-fitted $K_{3,3}$ bisubdivision $K$. Choose a perfect matching $M^{\prime}$ of $H$ such that $K$ is well-fitted to $M^{\prime}$. Let $A$ and $B$ be the colour classes of $G$ (and $H$ ). Let $a_{1}, a_{2}, a_{3}, b_{1}, b_{2}$, and $b_{3}$ be the vertices of degree three of $K$, where $\left\{a_{1}, a_{2}, a_{3}\right\} \subset A$ and $\left\{b_{1}, b_{2}, b_{3}\right\} \subset B$. For a path with ends in $A$ and $B$, we assume the end in $A$ is the origin. Let $T_{i j}$ be the 2-path of $K$ with origin $a_{i}$ and terminus $b_{j}$ for $i=1,2,3$ and $j=1,2,3$.
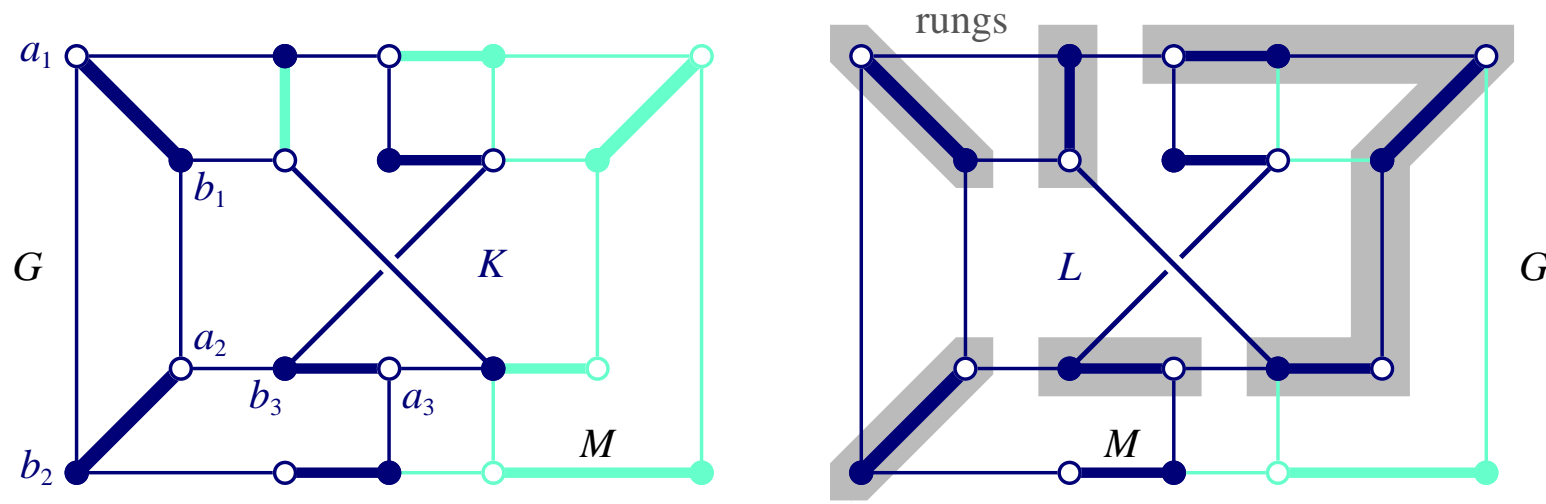

Figure 44: An example for Lemma 57. 
Suppose $e$ is an edge in $M$ which has an end on $K$ but is not in $E(K)$. Then $e$ is not in $M^{\prime}$ because $K$ is well-fitted to $M^{\prime}$. Hence, $e$ is on a cycle $C$ whose edges are alternately in $M$ and $M^{\prime}$. Let $P$ be the path of $C$ such that $e$ is on $P$, the ends of $P$ are on $K$, and no intermediate vertex of $P$ is on $K$. Let $\mathcal{P}$ be the set of all such paths $P$.

Note that every path in $\mathcal{P}$ is well-fitted to $M$ and is an $M^{\prime}$-ear path of $K$. Consequently, the paths in $\mathcal{P}$ are disjoint, and each has odd length. If $\mathcal{P}=\emptyset$, then $K$ is well-fitted to $M$ and we are done. Thus, we may assume there is a path $P$ in $\mathcal{P}$. Let $u$ and $v$ be the ends of $P$, where $u \in A$ and $v \in B$.

Suppose $u$ and $v$ are on some 2-path $T_{i j}$ of $K$. Let $K^{\prime}$ be the $K_{3,3}$ bisubdivision $\left(K-T_{i j}[u, v]\right)+P$ and let $f$ be the first edge of $T_{i j}[u, v]$. Lemma 26 can be used to show $K^{\prime}$ is well-fitted to $H$. Then $K^{\prime}$ is well-fitted to $H-f$ since $f$ is incident with $K^{\prime}$. Furthermore, $f$ is not in $M$ because it is adjacent to the first edge of $P$, which is in $M$. But now $H-f$ contradicts the minimality of $H$.

Suppose $u$ and $v$ are on two different 2-paths of $K$ which share an end. We may assume $u$ is on $T_{11}$ and $v$ is on $T_{12}$. Let $g$ be the last edge of $T_{12}\left[a_{1}, v\right]$. As in the previous paragraph, we can contradict the minimality of $H$ by showing $\left(K-T_{12}\left[a_{1}, v\right]\right)+P$ is a $K_{3,3}$ bisubdivision which is well-fitted to $H-g$.

Suppose $u$ and $v$ are intermediate vertices of $T_{i j}$ and $T_{k \ell}$, respectively, where $T_{i j}$ and $T_{k \ell}$ are disjoint. Let $K^{\prime \prime}$ be the $K_{3,3}$ bisubdivision $\left[K-T_{i \ell}\right]+P$. Lemma 26 can be used to show $K^{\prime \prime}$ is well-fitted to some perfect matching $M^{\prime \prime}$ of $H$. If $T_{i \ell}$ is not well-fitted to $M$, then $T_{i \ell}$ has edge $h$ which is not in $M \cup M^{\prime \prime}$. But then $H-h$ contradicts the minimality of $H$. Hence, $T_{i \ell}$ is well-fitted to $M$.

In the three previous paragraphs we have shown that the ends of a path $P$ in $\mathcal{P}$ are intermediate vertices of disjoint 2-paths of $K$, and that if the origin and terminus of $P$ are on $T_{i j}$ and $T_{k \ell}$, respectively, then $T_{i \ell}$ is well-fitted to $M$. We refer to this last fact as property $*$. Let $A_{12}$ be the intermediate vertices of $T_{12}$ which are origins of paths in $\mathcal{P}$, and let $B_{12}$ be the intermediate vertices of $T_{12}$ which are termini of paths in $\mathcal{P}$. Since $\mathcal{P}$ is nonempty, we may assume $A_{12}$ in nonempty.

Let $P$ be a path in $\mathcal{P}$ whose origin, $u$, is in $A_{12}$. We may assume the terminus of $P$ is an intermediate vertex of $T_{21}$. Then $T_{11}$ is well-fitted to $M$ by property $*$. Now consider $T_{12}\left[a_{1}, u\right]$. The first and last edges of this path are not in $M$. Furthermore, the number of intermediate vertices in $B$ on this path is one more than the number of intermediate vertices in $A$. Hence, some intermediate vertex of $T_{12}\left[a_{1}, u\right]$ is in $B_{12}$. Similarly, we can show that if $u^{\prime}$ is a vertex in $A_{12}$ distinct from $u$, then $T_{12}\left[u, u^{\prime}\right]$ has an intermediate vertex in $B_{12}$. Thus, every nontrivial subpath of $T_{12}$ with ends in $A_{12} \cup\left\{a_{1}\right\}$ has an intermediate vertex in $B_{12}$. Similarly, every nontrivial subpath of $T_{12}$ with ends in $B_{12} \cup\left\{b_{2}\right\}$ has an intermediate vertex in $A_{12}$. Therefore, there exist paths $P_{1}, \ldots, P_{2 p}$ in $\mathcal{P}$ (F. 45a), where $P_{i}$ has origin $u_{i}$ and terminus $v_{i}$ for $i=1, \ldots, 2 p$, such that $B_{12}=\left\{v_{1}, v_{3}, \ldots, v_{2 p-1}\right\}$, $A_{12}=\left\{u_{2}, u_{4}, \ldots, u_{2 p}\right\}$, and we have the order $a_{1}, v_{1}, u_{2}, v_{3}, u_{4}, \ldots, v_{2 p-1}, u_{2 p}, b_{2}$ on $T_{12}$.

We may assume $u_{1}$ is an intermediate vertex of $T_{21}$. We know the terminus $v_{2}$ of $P_{2}$ is an intermediate vertex of some 2-path of $K$ which is disjoint from $T_{12}$. Hence, $v_{2}$ is an intermediate vertex of $T_{21}\left[a_{2}, u_{1}\right], T_{21}\left[u_{1}, b_{1}\right], T_{31}, T_{33}$, or $T_{23}$. We will show the last four possibilities lead to a contradiction. 

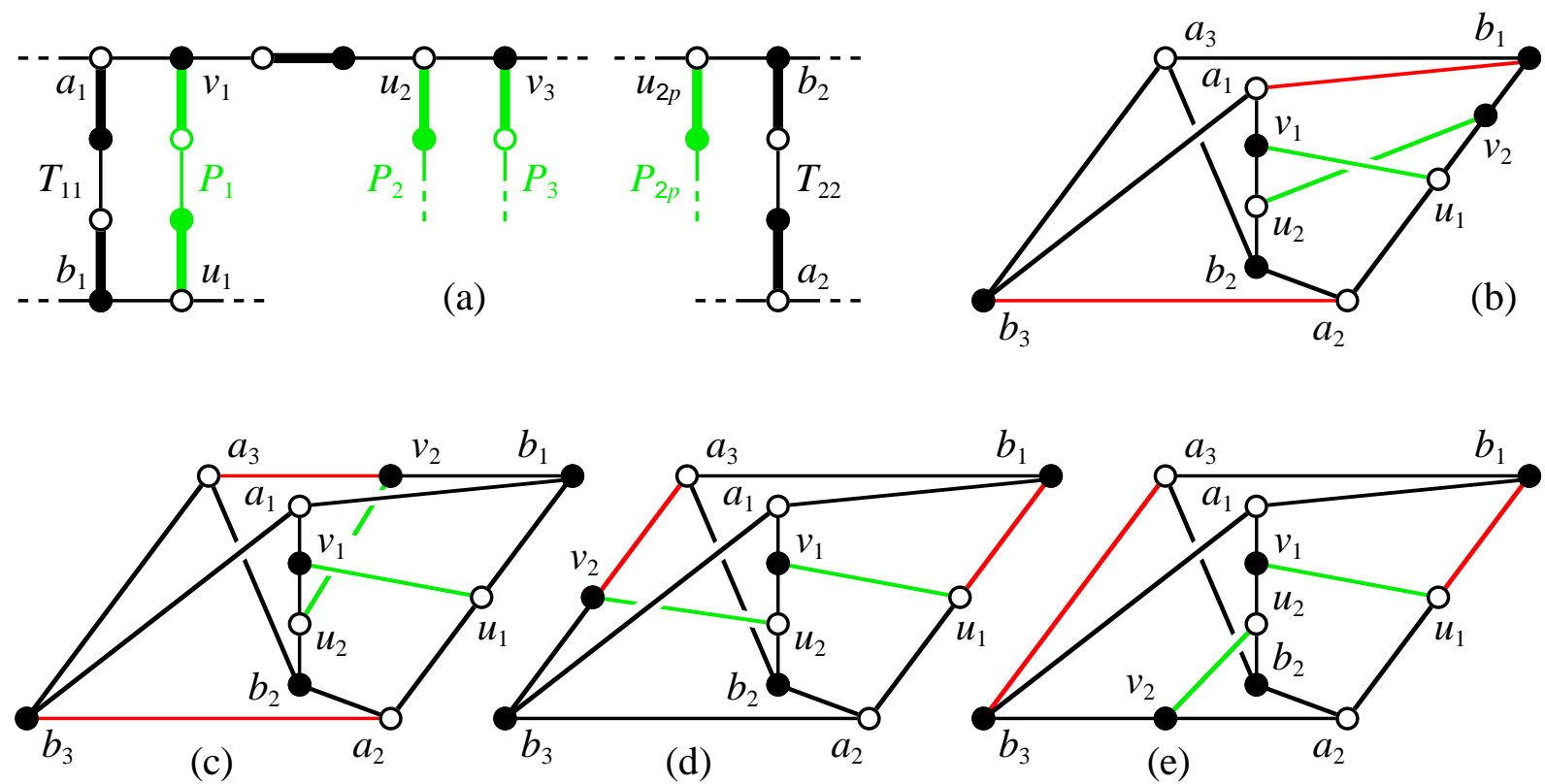

(f)

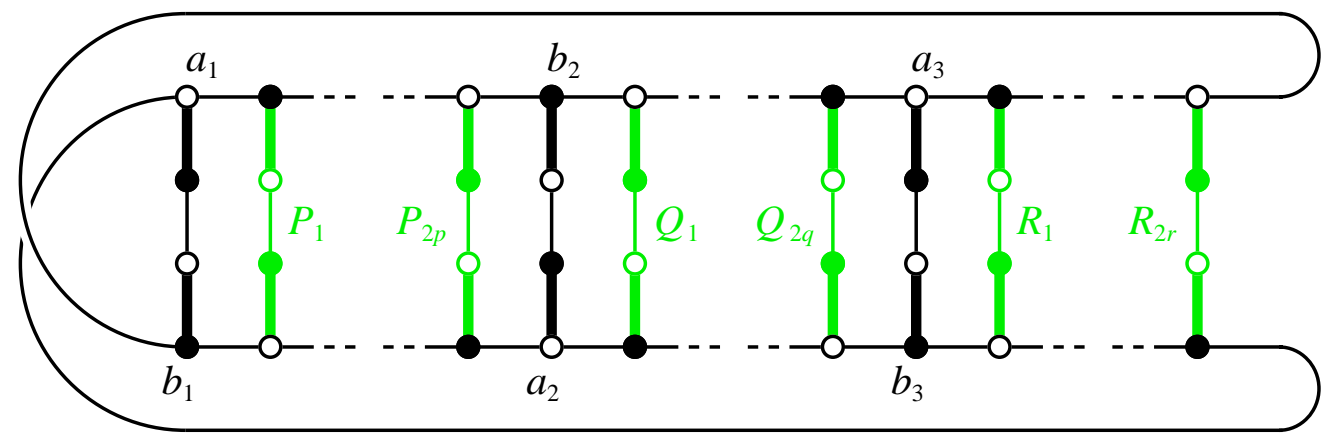

Figure 45: The proof of Lemma 57.

Suppose $v_{2}$ is on $T_{21}\left[u_{1}, b_{1}\right]$ (F. 45b). Lemma 26 can be used to show that the $K_{3,3}$ bisubdivision $\left[K-\left(T_{11}+T_{23}\right)\right]+P_{1}+P_{2}$ is well-fitted to a perfect matching $M^{\prime \prime}$ of $H$. Considering $P_{1}$, property $*$ implies $T_{22}$ is well-fitted to $M$. Hence, the first edge, $e$, of $T_{23}$ is not in $M \cup M^{\prime \prime}$. But then $H-e$ contradicts the minimality of $H$. We also contradict the minimality of $H$ when $v_{2}$ is an intermediate vertex of $T_{31}, T_{33}$, or $T_{23}$ (F. 45cde). Therefore, $v_{2}$ is an intermediate vertex of $T_{21}\left[a_{2}, u_{1}\right]$. Similarly, we can show that $u_{3}, v_{4}, \ldots, u_{2 p-1}, v_{2 p}$ are on $T_{21}$, and that we have the order $a_{2}, v_{2 p}, u_{2 p-1}, \ldots, v_{4}, u_{3}, v_{2}, u_{1}, b_{1}$ on $T_{21}$. As well, $P_{1}, \ldots, P_{2 p}$ are the only paths in $\mathcal{P}$ with an end on $T_{21}$.

Considering $P_{1}$ and $P_{2 p}$, property $*$ implies $T_{11}$ and $T_{22}$ are well-fitted to $M$. We have shown that the ends of any path in $\mathcal{P}$ are intermediate vertices of disjoint 2-paths of $K$. Hence, if there is a path in $\mathcal{P}$ with an end on $T_{33}$, then the other end of the path is on $T_{12}, T_{22}, T_{21}$, or $T_{11}$. But $T_{11}$ and $T_{22}$ are well-fitted to $M$, while $P_{1}, \ldots, P_{2 p}$ are the only paths in $\mathcal{P}$ with an end on $T_{12}$ or $T_{21}$. Thus, no path in $\mathcal{P}$ has an end on $T_{33}$. 
Suppose there is a path in $\mathcal{P}$ with ends on $T_{13}$ and $T_{32}$. Then by previous arguments there is a path in $\mathcal{P}$ with origin on $T_{13}$ and terminus on $T_{32}$. Property $*$ then implies $T_{12}$ is well-fitted to $M$. But then the existence of $P_{1}$ is contradicted. Therefore, no path in $\mathcal{P}$ has ends on $T_{13}$ and $T_{32}$. Similarly, no path in $\mathcal{P}$ has ends on $T_{31}$ and $T_{23}$. It follows that $\mathcal{P}=\left\{P_{1}, \ldots, P_{2 p}, Q_{1}, \ldots, Q_{2 q}, R_{1}, \ldots, R_{2 r}\right\}$, where $Q_{j}$ has ends on $T_{32}$ and $T_{23}$ for $j=1, \ldots, 2 q$, and $R_{k}$ has ends on $T_{13}$ and $T_{31}$ for $k=1, \ldots, 2 r$ (F. 45f). Arguments applied to $\left\{P_{1}, \ldots, P_{2 p}\right\}$ can be applied to $\left\{Q_{1}, \ldots, Q_{2 q}\right\}$ and $\left\{R_{1}, \ldots, R_{2 r}\right\}$ to show $K+\sum_{P \in \mathcal{P}} P$ is an $M_{4 n+2}$ bisubdivision which is well-fitted to $M$ for some $n \geq 2$. Also, its rungs, that is, the paths in $\mathcal{P} \cup\left\{T_{11}, T_{22}, T_{33}\right\}$, are all well-fitted to $M$.

Theorem 58 If $D$ is a digraph, and $G$ is the corresponding bipartite graph, then the following statements are equivalent.

a) $D$ does not have a negative $\{-1,1\}$-arc weighting.

b) $G$ does not have an unbalanced $\{-1,1\}$-edge weighting

c) D has a weak odd double dicycle.

d) $G$ has a well-fitted $K_{3,3}$ bisubdivision.

Proof $(\mathbf{a} \Leftrightarrow \mathbf{b})$. This follows from Lemma 5 and Theorem 4 .

(c $\Leftrightarrow \mathbf{d})$. Let $M$ be the perfect matching of $G$ corresponding to $V(D)$. Suppose $G$ has a well-fitted $K_{3,3}$ bisubdivision. By Lemma 57, $G$ has an $M_{4 n+2}$ bisubdivision $L$ which is well-fitted to $M$ for some $n \geq 1$, and furthermore, the rungs of $L$ are well-fitted to $M$ if $n \geq 2$. Then $L$ corresponds to a weak $\overleftrightarrow{C}_{2 n+1}$ subdigraph of $D$, where the vertex set of the weak $\overleftrightarrow{C}_{2 n+1}$ corresponds to the perfect matching $M \cap E(L)$ of $L$.

Conversely, suppose $D$ has a weak $\overleftrightarrow{C}_{2 n+1}$, for some $n \geq 1$. Then $G$ has an $M_{4 n+2}$ bisubdivision $L$ which is well-fitted to $M$. Lemma 26 can then be used to show that some subgraph of $L$ is a well-fitted $K_{3,3}$ bisubdivision of $G$.

$(\mathbf{d} \Rightarrow \mathbf{b})$. This is Theorem 12 in the "easy" direction, which we have already proven.

( $\mathbf{a} \Rightarrow[\mathbf{c}$ or $\mathbf{d}])$. We prove this by induction. It is trivial if $\nu(D) \leq 2$. Suppose $\nu(D) \geq 3$ and $D$ does not have a negative $\{-1,1\}$-arc weighting. If $D$ is not strongly connected, then some strong component of $D$ does not have a negative $\{-1,1\}$-arc weighting. By the induction hypothesis, this component has a weak odd double dicycle. Hence, the same is true for $D$. Thus, we may assume $D$ is strongly connected.

If $D$ is not strongly 2-connected, then $D$ has a vertex $v$ such that $D-v$ is not strongly connected. Let $D_{1}$ and $D_{2}$ be the reductions of $D$ at $v$. (See pages 23 and 24.) Then $D_{1}$ or $D_{2}$ does not have a negative $\{-1,1\}$-arc weighting by Lemma 8 . By the induction hypothesis, $D_{1}$ or $D_{2}$ has a weak odd double dicycle. It is then routine to show $D$ has a weak odd double dicycle. Thus, we may assume $D$ is strongly 2 -connected. Then $G$ is a brace by Theorem 3. Furthermore, $G$ does not have an unbalanced $\{-1,1\}$-edge weighting by the equivalence of (a) and (b). Hence, $G$ has a well-fitted $K_{3,3}$ bisubdivision by the Main Theorem. 


\section{Acknowledgements}

Robertson, Seymour, and Thomas [29, 37] independently proved the Main Theorem. The two proofs use different methods. The author wishes to thank Thomas Lundy for a helpful discussion, and Moshé Rosenfeld for suggesting that he look at a problem in which Victor Klee was interested.

\section{References}

[1] L. Bassett, J. Maybee, and J. Quick. Qualitative economics and the scope of the correspondence principle. Econometrica, 36:544-563, 1968.

[2] J. C. Bermond and C. Thomassen. Cycles in digraphs - a survey. J. Graph Theory, $5: 1-43,1981$.

[3] J. A. Bondy and U. S. R. Murty. Graph Theory with Applications. North-Holland, Amsterdam, 1981.

[4] R.A. Brualdi and H. Perfect. Extension of partial diagonals of matrices I. Monatshefte Math., 75:385-397, 1971.

[5] R.A. Brualdi and B.L. Shader. On sign-nonsingular matrices and the conversion of the permanent into the determinant. In Applied Geometry and Discrete Mathematics, number 4 in DIMACS Ser. Discrete Math. Theoret. Comput. Sci., pages 117-134. Amer. Math. Soc., Providence, 1991.

[6] R.A. Brualdi and B.L. Shader. Matrices of Sign-Solvable Linear Systems. Cambridge University Press, Cambridge, 1995.

[7] M.E. Fisher. Statistical mechanics of dimers on a plane lattice. Phys. Rev. (2), 124:1664-1672, 1961.

[8] G. Frobenius. Über matrizen aus nicht negativen elementen. Sitzungsber. König. Preuss. Akad. Wiss., 26:456-477, 1912.

[9] G. Frobenius. Über zerlegbare determinanten. Sitzungsber. König. Preuss. Akad. Wiss., XVIII:247-277, 1917.

[10] P. M. Gibson. Conversion of the permanent into the determinant. Proc. Amer. Math. Soc., 27:471-476, 1971.

[11] W. M. Gorman. A wider scope for qualitative economics. Rev. Econ. Studies, 31:65-68, 1964.

[12] D.J. Hartfiel. A simplified form for nearly reducible and nearly decomposable matrices. Proc. Amer. Math. Soc., 24:388-393, 1970. (erratum, page 1965). 
[13] G. Hetyei. Rectangular configurations which can be covered by $2 \times 1$ rectangles. Pécsi Tan. Föisk. Közl., 8:351-367, 1964. (in Hungarian).

[14] P. W. Kasteleyn. The statistics of dimers on a lattice. Physica, 27:1209-1225, 1961.

[15] P. W. Kasteleyn. Dimer statistics and phase transitions. J. Math. Phys., 4:287-293, 1963.

[16] P. W. Kasteleyn. Graph theory and crystal physics. In Graph Theory and Theoretical Physics, pages 44-110. Academic Press, New York, 1967.

[17] V. Klee, R. Ladner, and R. Manber. Signsolvability revisited. Linear Alg. and Appls, 59:131-157, 1984.

[18] K. Koh. Even circuits in directed graphs and Lovász' conjecture. Bull. Malayasian Math. Soc., 7:47-52, 1976.

[19] D. König. Line systems and determinants. Math. Termész. Ért., 33:221-229, 1915. (in Hungarian).

[20] D. König. Über graphen und ihre andwendung auf determinantentheorie und mengenlehre. Math. Ann., 77:453-465, 1916.

[21] K. Lancaster. The scope of qualitative economics. Rev. Econ. Studies, 29:99-132, 1962.

[22] K. Lancaster. Partitionable systems and qualitative economics. Rev. Econ. Studies, 31:69-72, 1964.

[23] K. Lancaster. The theory of qualitative linear systems. Econometrica, 33:395-408, 1964.

[24] R. Levow. Counterexamples to conjectures of Ryser and de Oliveira. Pacific J. Math., 44(2):591-603, 1973.

[25] C.H.C. Little. A characterization of convertible (0,1)-matrices. J. Combin. Theory, Ser. B, 18:187-208, 1975.

[26] L. Lovász. Problem 2. In Recent Advances in Graph Theory, Proc. Symp. Prague 1974, Prague, 1975. Academia Praha.

[27] L. Lovász and M. D. Plummer. On minimal elementary bipartite graphs. J. Combin. Theory, Ser. B, 23(1):127-138, 1977.

[28] L. Lovász and M. D. Plummer. Matching Theory. Elsevier, Amsterdam, 1986.

[29] W. D. McCuaig, N. Robertson, P. D. Seymour, and R. Thomas. Permanents, Pfaffian orientations, and even directed circuits. In Proc. ACM Symp. Theory Comput., pages 402-405, May 1997. 
[30] William McCuaig. Even dicycles. J. Graph Theory, 35:46-68, 2000.

[31] William McCuaig. Brace generation. J. Graph Theory, 38:124-169, 2001.

[32] K. Menger. Zur allgemeinen kurventheorie. Fund. Math., 10:96-115, 1927.

[33] R.T. Morrison and R.N. Boyd. Organic Chemistry. Allyn and Bacon, Toronto, third edition, 1973.

[34] M. D. Plummer. On n-extendable graphs. Discrete Math., 31:201-210, 1980.

[35] M. D. Plummer. Matching and vertex packing: How "hard" are they? In Quo Vadis, Graph Theory, number 55 in Annals of Discrete Mathematics, pages 275-312, Amsterdam, 1993. North-Holland.

[36] G. Pólya. Aufgabe 424. Arch. Math. Phys., 20:271, 1913.

[37] N. Robertson, P. D. Seymour, and R. Thomas. Permanents, Pfaffian orientations, and even directed circuits. Ann. of Math. (2), 150(3):929-975, 1999.

[38] P.A. Samuelson. Foundations of Economic Analysis. Atheneum, New York, 1971.

[39] T. J. Schaefer. The complexity of satisfiability problems. In Proc. Tenth ACM Symp. Theory Comput., pages 216-226, 1978.

[40] P. D. Seymour. Master's thesis, Oxford University, 1973.

[41] P. D. Seymour. On the two-colouring of hypergraphs. Quart. J. Math. Oxford, 25:303-312, 1974.

[42] P. D. Seymour and C. Thomassen. Characterization of even directed graphs. J. Combin. Theory, Ser. B, 42:36-45, 1987.

[43] G. Szegö. Lösung zu 424. Arch. Math. Phys., 21:291-292, 1913.

[44] H.N.V. Temperley and M.E. Fisher. Dimers problem in statistical mechanics - an exact result. Philos. Mag. (8), 6:1061-1063, 1961.

[45] C. Thomassen. Sign-nonsingular matrices and even cycles in directed graphs. Linear Alg. Appls., 75:27-41, 1986.

[46] C. Thomassen. The even cycle problem for planar directed graphs. J. Algorithms, 15:61-75, 1993.

[47] M. F. Tinsley. Permanents of cyclic matrices. Pacific J. Math., 10:1067-1082, 1960.

[48] W. T. Tutte. Graph Theory. Addison-Wesley, Don Mills, Ontario, 1984.

[49] L. G. Valiant. The complexity of computing the permanent. Theoretical Computer Science, 8:189-201, 1979. 